\title{
Narrow Bracketing and Dominated Choices ${ }^{1}$
}

\author{
Matthew Rabin \\ Department of Economics \\ University of California - Berkeley \\ and \\ Georg Weizsäcker \\ Department of Economics \\ London School of Economics and Political Science
}

\begin{abstract}
An experiment by Tversky and Kahneman (1981) illustrates that people's tendency to evaluate risky decisions separately can lead them to choose combinations of choices that are first-order stochastically dominated by other available combinations. We investigate the generality of this effect both theoretically and experimentally. We show that for any decisionmaker who does not have constant-absolute-risk-averse preferences and who evaluates her decisions one by one, there exists a simple pair of independent binary decisions where the decisionmaker will make a dominated combination of choices. We also characterize, as a function of a person's preferences, the amount

\footnotetext{
${ }^{1}$ We are grateful to Dan Benjamin, Syngjoo Choi, Erik Eyster, Thorsten Hens, Michele Piccione, Peter Wakker, Heinrich Weizsäcker and seminar participants at Caltech, Amsterdam, Helsinki, IIES Stockholm, IZA Bonn, LSE, Nottingham, Zurich and the LEaF 2006 conference for helpful comments, and to Zack Grossman and Paige Marta Skiba for research assistance. The survey experiment was made possible by the generous support of TESS (Time-Sharing Experiments in the Social Sciences) and the efforts by the staff of Knowledge Networks. We also thank the ELSE Centre at University College London for the generous support of the laboratory experiment, and Rabin thanks the National Science Foundation for financial support.
} 
of money that she can lose due to a single mistake of this kind. The theory is accompanied by both a real-stakes laboratory experiment and a large-sample survey from the general U.S. population. Replicating Tversky and Kahneman's original experiment where decisionmakers with prototypical prospect-theory preferences will choose a dominated combination, we find that $28 \%$ of the participants do so. In the survey we ask the respondents about several hypothetical large-stakes choices, and find higher proportions of dominated choice combinations. A statistical model that estimates preferences from the survey results is best fit by assuming people have utility functions that are close to prospect-theory value functions and that about $83 \%$ of people bracket narrowly. None of these results varies strongly with the personal characteristics of participants. We also demonstrate directly that dominated choices are driven by narrow bracketing: when we eliminate the possibility of narrow bracketing by using a combined presentation of the decisions, the dominated choices are eliminated in the laboratory experiment and are greatly reduced in the survey.

Keywords: Lottery choice, narrow framing, representative-sample experiments

JEL Classification: B49

Contact: rabin@econ.berkeley.edu, g.weizsacker@lse.ac.uk 


\section{Introduction}

Tversky and Kahneman (1981) present an experiment that demonstrates an adverse effect of "narrow bracketing": a decisionmaker who faces multiple decisions and chooses an option in each case without regard to the other decisions that she faces is prone to make a dominated combination of choices that is inconsistent with any reasonable preferences. The experiment show that people make this error when faced with only a pair of independent simple binary decisions that are presented on the same sheet of paper. In our slight reformulation, subjects were presented with the following:

You face the following pair of concurrent decisions. First examine both decisions, then indicate your choices, by circling the corresponding letter. Both choices will be payoff relevant, i.e. the gains and losses will be added to your overall payment.

Decision (i): Choose between
A. a sure gain of $£ 2.40$
B. a $25 \%$ chance to gain $£ 10.00$ and a $75 \%$ chance to gain $£ 0.00$.

Decision (ii): Choose between

C. a sure loss of $£ 7.50$

D. a $75 \%$ chance to lose $£ 10.00$, and a $25 \%$ chance to lose $£ 0.00$.

If, as predicted by Kahneman and Tversky's (1979) prospect theory, the decisionmaker is riskaverting in gains and risk-seeking in losses and if she applies these preferences separately to the decisions, then she will tend to choose $A$ and $D$. This prediction was confirmed: $60 \%$ of Tversky and Kahneman's (1981) participants chose this combination with small real stakes, and $73 \%$ did so for large hypothetical stakes. But $A$ and $D$ is first-order stochastically dominated: the joint distribution resulting from the combination of $B$ and $C$ is a $\frac{1}{4}$ chance of gaining $£ 2.50$ and a $\frac{3}{4}$ chance of losing $£ 7.50$; the joint distribution of $A$ and $D$ is a $\frac{1}{4}$ chance of gaining $£ 2.40$ and a $\frac{3}{4}$ chance of losing $£ 7.60$. The $B C$ combination is equal to the $A D$ combination plus a sure payoff 
of $£ 0.10$. Our experiments — laboratory experiments both with real and hypothetical payments, and a hypothetical-payment survey experiment with a representative sample from the general U.S. population - confirm the pattern of frequent $A D$ choices, although at a somewhat lower level: With large hypothetical payoffs (the above amounts multiplied by 100 ) about $60 \%$ of participants choose $A D$; for small real stakes, $28 \%$ of the subjects do so. (And $34 \%$ do so for small hypothetical payments.) In the survey experiment we also find that between $40 \%$ and $50 \%$ of subjects make dominated choices in three other large-stakes examples of sets of decisions, despite giving up larger (hypothetical) amounts than in the original example $-\$ 50$ or $\$ 75$ in expectation, rather than $\$ 10 .^{2}$

These dominance violations are a demonstration of narrow bracketing, because the choice of $A D$ is clearly due to the separate presentation. ${ }^{3}$ Theoretically, we are not aware of any utility theory ever proposed that would allow for the choice of $A D$ over $B C .{ }^{4}$ Empirically, almost no decisionmaker presented with the explicit choice between $A D$ and $B C$ would choose $A D$, as is confirmed by our experiments where the violation rates under such a "broad presentation" treatment are reduced to $0 \%$ and $6 \%$, respectively, in the laboratory and the survey. ${ }^{5}$

\footnotetext{
${ }^{2}$ Our instructions also made explicit that all random draws were independent, alleviating concerns that Tversky and Kahneman's results might be due to this ambiguity.

${ }^{3}$ A related example of narrow bracketing is Redelmeier and Tversky's (1992) demonstration that the investment choice in a risky asset can depend on whether the asset is framed as part of a portfolio of other assets or as a standalone investment. See also the replication and variation in Langer and Weber (2001), and the literature cited there. Other evidence on narrow bracketing in lottery choice include Gneezy and Potters (1997) and Thaler et al (1997) who test whether mypoic loss aversion - a form of narrow bracketing - may serve as as a possible explanation of the equity-premium puzzle, and by Camerer (1989) and Battalio et al (1990), both of whom present treatment variations that suggest narrow bracketing of standard lottery choices. Papers that have explored the principles of what we call narrow bracketing include Kahneman and Lovallo (1993), Benartzi and Thaler (1995) and Read, Loewenstein, and Rabin (1999).

${ }^{4}$ Even models that allow for dominance violations - such as the disappointment-theory models of Bell (1985) and Loomes and Sugden (1986), the related "choice-acclimating personal equilibrium" concept in Köszegi and Rabin (2007), as well as models with a taste for gambling such as Diecidue, Schmidt, and Wakker (2004) - do not permit the preference for $A D$ over $B C$, since $B C$ is simply $A D$ plus a sure amount of money.

${ }^{5}$ We examine the broad-bracketed presentation in three examples altogether in the survey experiment (see Section 3 ), and the violation rates are reduced from $66 \%$ to $6 \%$, from $40 \%$ to $3 \%$, and from $50 \%$ to $29 \%$. The surprisingly high violations of dominance in the last example even under broad presentation are inconsistent with any hypothesis
} 
The theoretical part of the paper contributes to understanding the generality with which narrow bracketing can lead to dominated choices. Diecidue and Wakker (2002) follow de Finetti (1974) in showing that unless a narrow-bracketing decisionmaker's preferences exhibit risk neutrality, there exists a set of correlated gambles such that her combined choice will be dominated by another feasible combination of choice. In this light, the insight from Tversky and Kahneman's example is that even if a prospect-theoretic decisionmaker merely ignores other choices that involve uncorrelated events, she may choose a first-order stochastically dominated portfolio. In Section 2, we generalize this in several ways. The main result establishes that the basic logic of Tversky and Kahneman's example extends quite broadly beyond prospect-theoretic preferences: if a narrow-bracketing decisionmaker's risk attitudes are not identical at all possible ranges of outcomes — essentially, if she does not have constant-absolute-risk-aversion (CARA) preferences - then there exists a pair of binary choices where she chooses a dominated combination.

The logic behind this simple result is itself simple. In the Tversky and Kahneman example, a prospect-theoretic chooser changes from risk averse over a prospect in the gain domain to riskloving over the shifted prospect that lies in the loss domain. When narrowly bracketing, she takes a less-than-expected-value certain amount over the lottery in the gain domain but takes the lottery over its expected value in the loss domain. She'd be better off doing the opposite. But the potential for dominance depends neither on switches between risk aversion and risk-lovingness nor on where or how her risk attitudes differ: if a person's risk attitudes are not identical over all possible ranges, then there always exists a lottery such that the distance between the lottery's expected value and the lottery's certainty equivalent will be different for different outcome ranges. Hence, there exists a non-risky alternative payment that a person would prefer to a given lottery in one range and this payment is less attractive than a non-risky payment that she would reject in favor of the same lottery in another range. If she is a narrow bracketer and is offered both decisions between such non-risky payments and the corresponding lotteries, then she will implement these two "narrowly preferred" choices. But since her actual payoff is the sum of the two payoffs, reversing her choices

about choice behavior that we are aware of and we do not understand what motives were induced by the design of this example. 
leaves her with the same risk but a higher distribution of outcomes.

The argument also makes no assumption that the decisionmaker is an expected-utility maximizer in the sense of weighting prospects linearly in probabilities, but follows solely from monotonicity and completeness of preferences, plus the assumption that a certainty equivalent exists. In Section 2 we also establish two stronger results for the case that the narrowly-bracketing decisionmaker evaluates prospects linearly in probabilities. First, we characterize as a function of preferences a lower bound on the maximum amount that the decisionmaker can leave on the table in only two choices. This characterization implies that a narrow bracketer with preferences substantially different from CARA can be made to give up substantial amounts of money in only two choices between uncorrelated gambles. Second, we show that even for a decisionmaker who has an arbitrarily small propensity to narrowly bracket (measured in a particular way) there exists a pair of simple binary choice problems such that she would violate dominance.

These results establish the existence of situations that generate dominance violations, but not the empirical prevalence of such situations or scale of welfare loss from dominated choices. Indeed, the fact that different pairs of binary choices are needed to "catch" decisionmakers with different preferences means that any given pair of choices might induce dominance in only a small proportion of the population. On the other hand, any non-CARA narrow bracketer who faces a large enough set of varied choices will make a dominated choice overall if only one pair of those choices generates a dominated choice, and she will suffer a welfare cost that increases with the frequency of dominanted choices. Further, it is important to note that while dominance violations are a clear example of suboptimal behavior, there are many other ways in which an agent will fail to maximize her utility if she does not incorporate multiple choices into a combined problem. We focus on dominance violations because these are known to be mistakes for all monotonic preferences. ${ }^{6}$

However, while the high rates of dominance violations in the experiments indicate that subjects are not broad-bracketing utility maximizers, this does not per se demonstrate that narrowbracketing utility maximization has much more explanatory power. We therefore jointly estimate

\footnotetext{
${ }^{6}$ While all of our experimental examples involve the possibly not-too-prevalent case of lotteries that lie fully in the loss domain, a corollary of our main result is that if a person does not have CARA preferences over all lotteries involving a chance of gain, she will be susceptible to dominated choices even when all risks involve gains.
} 
the subjects' utility and the pervasiveness of narrow bracketing, using a simple statistical model where all agents are assumed to maximize their utility but where narrow-bracketing and broadbracketing agents co-exist. The results reinforce the dominance findings, suggesting that narrow bracketing is frequent and that subjects exhibit largely coherent preferences given those narrow brackets. We estimate that about five out of six decisions are made with narrow brackets, and that the shape of the participants' utility function (assumed to be the same for both types of agents) is reminiscent of Prospect Theory's value function, with risk aversion in gains and around the status quo point, and a preference for risk in losses. An important caveat is that such estimations come with specific (albeit flexible) functional form assumptions - in particular that preferences are homogeneous across the population. However, any hypothetical distribution of broad-bracketed preferences in the population would run into difficulties with explaining several data patterns: (i) the observed cases of dominance violations (ii) the strong effects of broad versus narrow framing, (iii) apparent shifts in the degree of risk aversion - there are some modal choices that broad bracketers would only make if they had a preference for risk in the gains domain, and other choices that suggest wide-spread risk aversion. Moreover, we find that the "standard" expected-utility model - which prescribes very mild but broad-bracketed risk aversion for all choices - performs really poorly in our data: its prediction is observed in only few percent of the cases, below the level that is predicted by assuming pure noise in all decisions. Even imposing homogeneity of preferences, the estimated narrow model organizes the data better, correctly predicting the modal choice frequencies in the majority of our experimental treatments.

The representative nature of the survey sample combined with the data on personal characteristics allows us to ask who brackets narrowly. We find few strong correlations with observable background characteristics, so that the main results hold about equally for almost all groups of respondents. Re-estimating the model for different subgroups separately, we find that in each examined subgroup fewer than $22 \%$ of people are broad bracketers. But while the degree of bracketing is small for all subgroups, there are a number of differences in their preferences. Respondents in the "non-white" ethnic/racial category are estimated to be more risk-neutral with respect to lotteries around zero and in the gains domain. This corresponds to a significant observed behavioral differ- 
ence: non-whites are less likely to violate dominance. Other differences appear - e.g. the estimates suggest more risk neutral preferenecs for men than for women, and likewise between math-skilled and less math-skilled respondents - but these differences are less pronounced and do not correspond to reliable differences in violation rates. Perhaps most surprisingly, we find no significant effect of education on the violation rates. ${ }^{7}$

Taken together, our experimental and theoretical results illustrate the tension between assessments of the positive versus the normative status of different models of risky choice: narrow bracketing appears to be prevalent in decisions under risk, but will lead to decisions that are bad. Rabin (2000a) formalizes a common intuition among researchers that the conventional diminishingmarginal-utility-of-wealth expected-utility model not only fails in systematic ways to empirically describe risk behaviors, but cannot even in theory provide a calibrationally plausible account of modest-scale risk aversion. As suggested in Rabin (2000a, 2000b) and Rabin and Thaler (2001), and brought into further focus by Cox and Sadiraj (2006) and Rubinstein (2006), the culprit in the failure of the conventional model to account for departures from modest-scale risk neutrality is the premise that choice is determined by final wealth: maintaining the assumption that people's weighting of prospects are linear in probabilities, the reality of widespread modest-scale risk aversion could be accounted for by assuming as in Prospect Theory that changes in wealth are the carriers of value. ${ }^{8}$ Yet we note that the existing evidence of preferences over changes in wealth is essentially evidence of preferences over choice-by-choice isolated changes in wealth. As our new evi-

\footnotetext{
${ }^{7}$ The violation rate of respondent in the upper half of the income distribution is almost identical to that of the less rich respondents. The respondents who correctly completed our set of three mathematical questions have a $9 \%$ lower violation rate than the remaining respondents. Respondents with a bachelor's degree have a $5 \%$ higher violation rate than those with lower level of schooling. Respondents who report to have attended a math course in college have a $8 \%$ higher violation rate. None of these difference is significant at 5 percent in logistic regressions.

${ }^{8}$ Indeed, papers such as Benartzi and Thaler (1995), Bowman, Minehart, and Rabin (1999), Kőszegi and Rabin (2007) and many others have over the years emphasized that much of the insight of prospect theory as an alternative to conventional expected-utility analysis can be gleaned even assuming linear-probability preferences. Safra and Segal (2005) make the case even more clearly that the assumption that final wealth is the carrier of utility is the culprit: they show that a wide range of models that allow non-linear probability weighting but assume final wealth as the carrier of utility cannot provide plausible accounts of modest-scale risk aversion. Barberis, Huang and Thaler (2006) give a closely related discussion in a different model.
} 
dence confirms, most decisionmakers do not integrate their experimental choices with other choices that occur even within the same experimental session, and certainly not with simultaneous risks outside the experiment. Because the typical decisionmaker narrowly brackets preferences that do not have a constant degree of abslolute risk aversion, one can conclude that she is apt to make combinations of choices that are dominated. Along with other, less dramatic forms of mistakes, this reinforces Wakker's (2005) observation that choice-by-choice consistency with von Neumann's and Morgenstern's axioms does not per se yield the features of rationality generally associated with "expected-utility theory".

Section 5 concludes the paper with a brief discussion of whether such violations may be observed in markets where several agents interact, as well as some methodological implications for asessments of risk preferences.

\section{Theory}

Assume that the world consists of decisions $i=1, \ldots, I$ that are to be made simulateneously, with $I$ different choice sets $M_{1}, \ldots, M_{I}$. Denote the choice made in choice set $M_{i}$ by $m_{i}$. Each possible $m_{i} \in M_{i}$ induces a lottery, or probability distribution $L_{i}\left(x_{i} \mid m_{i}\right)$ over changes in wealth $x_{i} \in \mathbb{R}$ of the agent. We restrict attention to the case that the lotteries $L_{i}$ are independent between the "brackets" $i=1, \ldots, I{ }^{9}$ The vector of choices $m=\left(m_{1}, m_{2}, \ldots, m_{I}\right)$ induces a probability distribution over the sum of wealth changes $x^{I}=\sum_{i} x_{i}$, denoted by $F\left(x^{I} \mid m\right)$.

Following the terminology in Read, Loewenstein, and Rabin (1999), we assume that the decisionmaker is a narrow bracketer, meaning that she chooses within each of the $I$ choice sets according to her preferences over the outcomes but without consideration to the fact that the relevant outcome will be the combined outcome from all her choices. I.e. she evaluates the lotteries $L_{i}\left(x_{i} \mid m_{i}\right)$ but not the summed distribution $F\left(x^{I} \mid m\right)$. We assume further that the decisionmaker's preferences are complete and strictly monotonic over the set of all possible lotteries, and that in her

\footnotetext{
${ }^{9}$ As noted in the introduction, it is clear that non-risk-neutral narrow bracketers can choose dominated combinations if they are presented with correlated gambles. E.g. a risk-loving narrow bracketer may be tricked into paying a premium for entering two bets on the same coin throw, once on Heads and once on Tails.
} 
narrow evaluation, certainty equivalents exist for all lotteries. Because preferences are monotonic, the agent will never choose a dominated lottery within any single bracket — but the resulting distribution $F\left(x^{I} \mid m\right)$ may be dominated. To define the size of a first-order stochastic dominace (FOSD) violation, we say that $F_{1}$ dominates $F_{2}$ by an amount $\delta$ if it holds for all $x$ in their support that $F_{1}(x+\delta) \leq F_{2}(x)$. The decisionmaker violates FOSD by an amount $\delta$ if she chooses a distribution $F_{2}$ that is FOS-dominated by another available distribution $F_{1}$ by amount $\delta$. This measure $\delta$ has a straightforward interpretation: if the chosen $F_{2}$ is dominated by an amount $\delta$, then the decisionmaker could have chosen a distribution that is at least as desirable (from a broad-bracket perspective) as receiving both $F_{2}$ and a sure payment of $\delta$.

The propositions below state that lower bounds for the largest possible $\delta$ are linked to the decisionmaker's variability of risk attitudes, which can be captured by describing certainty equivalents and their variability. Let $L$ be a lottery with expected value $\mu_{L}$. Denote by $\widetilde{L} \equiv L+\triangle x$ a lottery that is generated by adding $\triangle x$ to all payoffs in $L$, keeping the probabilities constant: $\widetilde{L}$ is a shifted version of $L$. Finally, define the decisionmaker's net certainty equivalent for lottery $L, N C E_{L}$, as the difference between the (gross) certainty equivalent $C E_{L}$ - the sure payment that makes her indifferent to accepting $L$ - and the expected value of $L$ :

$$
N C E_{L}=C E_{L}-\mu_{L}
$$

$N C E_{L}$ is a measure of risk aversion towards the lottery $L$. A lower $N C E_{L}$ corresponds to a higher degree of risk aversion, and the agent is risk neutral if $N C E_{L}=0$.

Proposition 1 establishes that a decisionmaker will violate dominance in joint decisions to the degree that a shift can induce a change in the net certainty equivalent:

Proposition 1: Suppose that there exist shifted lotteries $L$ and $\widetilde{L}$, such that $\mid N C E_{L}-$ $N C E_{\widetilde{L}} \mid>\delta$. Then there exists a pair of binary choices such that the decisionmaker violates 
FOSD by the amount $\delta$.

All proofs are in Appendix 1.

The proof of the result strictly follows the construction of Tversky and Kahneman's example, where prospect theory predicts that the shift from negative-domain option $D$ to positive domain option $B$ generates a strong difference in the agent's risk aversion. The proposition clarifies that it is not necessary that the degree of risk aversion changes its sign as the lottery is shifted or that the lottery be of the kind that Tversky and Kahneman used. Most importantly, the construction works whenever the net certainty equivalent changes for any shift of any lottery. Not only does this mean that dominance can result from narrow bracketing for non-prospect-theory prefrences, but that it can result for all but a very particular class of preferences: e.g. for EU-representable preferences the proposition implies that a dominance violation is possible for preferences that can be represented by a utility function $v$ if and only if $v$ is not from the constant-absolute-value family of utility functions, where $v$ can be expressed as $v(x)=C A R A(x ; \beta, \alpha, r) \equiv \beta-\alpha \exp (-r x)$, for any $(\beta, \alpha, r) \in \mathbb{R}^{3}$. This is because the CARA family encompasses exactly those utility functions where the net certainty equivalent is constant for all shifts of all lotteries $L$. But the proof of Proposition 1 does not rely on preferences being representable, and hence the violations can occur even for a large class of non-EU preferences, as long as the decisionmaker narrowly brackets.

For any given $\delta$, however, Proposition 1 is silent about the set of preferences for which there is a pair of lotteries $L$ and $\widetilde{L}$ with the property $\left|N C E_{L}-N C E_{\widetilde{L}}\right|>\delta$. To investigate when decisionmaker is in danger of making a mistake of substantial size, we now consider preferences that are representable by a (possibly reference-dependent) strictly increasing function $v$ whose expected value she maximizes - but with a narrow, bracket-by-bracket evaluation of the available prospects. This allows to characterize a lower bound for the size of possible dominance violations, 
by comparing preferences to the CARA family. To do so, we define a metric for this comparison:

Definition: For an interval $[\underline{x}, \bar{x}] \subset \mathbb{R}$ of changes in wealth,

$$
K \equiv \inf _{(\beta, \alpha, r)} \max _{y \in[v(\underline{x}), v(\bar{x})]}\left|v^{-1}(y)-C A R A^{-1}(y ; \beta, \alpha, r)\right|
$$

is the horizontal distance between $v$ and the family of CARA functions.

That is, for an interval $[\underline{x}, \bar{x}], K$ is the smallest number such that all CARA functions are further or equally far away in $x$-direction somewhere on the interval. We can now state another simple proposition (although with a long proof):

Proposition 2: Suppose that $v$ is strictly increasing and continuously differentiable and has a horizontal distance of $K$ from the CARA family on the interval $[\underline{x}, \bar{x}]$. Then for all $\epsilon>0$ there exists a pair of binary choices - each between a binary lottery and a sure payment, and using only payoffs in $[\underline{x}, \bar{x}]$ — such that a narrow bracketer with preferences represented by $v$ violates FOSD by an amount greater than $K-\epsilon$.

In the above definition, $K$ indicates the change in risk attitudes across different ranges within $[\underline{x}, \bar{x}]$ - CARA represents a constant risk attitude, and $K$ measures the distance between $v$ and CARA. Proposition 2 shows that there is a straightforward way to find an example where narrow bracketing causes the decisionmaker to leave $K$ on the table. The proof provides a construction of two candidate lotteries $L^{A}$ and $L^{B}$, where at least one of them can always be shifted in a way that yields the violation. Further, the proposition ensures that these lotteries need involve only two outcomes and lie fully within $[\underline{x}, \bar{x}]$.

$K$ is defined conditional not only on $v$, but also on the interval $[\underline{x}, \bar{x}]$. When the interval is expanded, $K$ (weakly) increases, and indeed is likey to become infinitely large as the interval increases. This is true, e.g., if $v$ is a two-part linear function with a kink at some $x$-value or if $v$ is a constant relative risk aversion function (CRRA).

Our final theoretical result considers the question whether only fully narrow bracketer are in danger of falling into such traps. The answer is no, and once again it is a construction similar 
to Tversky and Kahneman's example that delivers the result. ${ }^{10}$ However, to capture the relative importance of broad versus narrow thinking, we now need to impose some additional structure on the preferences. A convenient formulation is the global-plus-local functional form of Barberis and Huang (2004) and Barberis, Huang and Thaler (2006): We assume that the agent's choices are determined by maximizing, over possible choice vectors $m$, the expression

$$
U(m)=\kappa \int u\left(x^{I}\right) d F\left(x^{I} \mid m\right)+(1-\kappa) \sum_{i} \int u\left(x_{i}\right) d L_{i}\left(x_{i} \mid m_{i}\right) .
$$

Here $u$ is a valuation function for money, which the decisionmaker applies both globally — evaluating total earnings, in the first term - and locally to each choice set $M_{i}$, in the second term. Notice that each element $m_{i}$ of $m$ enters $U$ in two ways: by contributing to the distribution $F$ of total wealth changes and through the narrow evaluation of payoffs in bracket $i$ alone. The parameter $\kappa$ is the weight of the global part, and we assume that $\kappa \in[0,1)$, so that the decisionmaker exhibits some degree of narrow bracketing. When $\kappa \rightarrow 1$, choices correspond to fully broad mazimization, and when $\kappa=0$, there is fully narrow bracketing. The proposition shows that if $u$ is different from CARA, then even mild degrees of narrow bracketing put the decisionmaker in danger of FOSD violations $-\kappa$ could be arbitrarily close to $1 .^{11}$

Proposition 3: Let $u$ be strictly increasing and twice continuously differentiable everywhere, and suppose that $u$ is not a member of the CARA family of functions. Then there is a pair of binary choices, each between a 50/50 lottery and a sure payment, where the decisionmaker violates FOSD.

In the proof of Proposition 3, it is shown that one can construct values $\left\{x_{1}, x_{2}, y_{1}, y_{2}, z\right\}$ with $y_{2}>y_{1}$, such that the agent will make the following pair of lottery choices:

Reject gamble 1: A 50/50 gamble between $x_{1}-y_{1}$ and $x_{1}+z$, with $y_{1}, z>0$, versus $x_{1}$ for sure.

\footnotetext{
${ }^{10}$ The result is even slightly simpler than in the original example, because it only uses $50 / 50$ lotteries.

${ }^{11}$ The proof in the appendix covers a somewhat stronger statement, allowing for different valuation functions in the broad versus narrow parts of the valuations. I.e. the proposition holds even if the function representing the broad valuation (here, $\kappa u$ ) is equal to CARA. It suffices if the narrow valuation function (here, $(1-\kappa) u)$ differs from CARA.
} 
Accept gamble 2: A 50/50 gamble between $x_{2}-y_{2}$ and $x_{2}+z$, with $y_{2}, z>0$, versus $x_{2}$ for sure.

Due to $y_{2}>y_{1}$, the choice combination $\left(\right.$ reject $_{1}$, accept $\left.t_{2}\right)$ is dominated by $\left(\right.$ reject $_{2}$, accept $\left._{1}\right)$. The assumption that $u$ is different from CARA is sufficient to identify two values $x_{1}$ and $x_{2}$ such that the function's local degree of risk aversion is larger at $x_{1}$ than at $x_{2}$. This can be used to show that an appropriate set of small values $y_{1}, y_{2}$, and $z$ can be found, with the above properties.

All three propositions highlight the role of constant absolute risk aversion. In fact, if most people's preferences were approximately CARA, then the typical decisionmaker would not make dominated choices. ${ }^{12}$ There is certainly no strong evidence for prevalence of CARA preferences in the literature (except for the special case of risk neutrality) but in the context of our discussion it is important to note that all estimates of risk attitudes will crucially depend on the maintained assumptions about bracketing. ${ }^{13}$ We are not aware of a study that simultaneously describes risk attitudes and narrowness of bracketing, and will provide such an estimation in the following sections.

\section{Experimental Design}

We conducted two experiments in different formats: One laboratory experiment that replicates and systematically varies the Tversky and Kahneman experiment ("Example 1", hereafter), and one survey experiment with a large and representative subject pool, where we introduce additional tasks. We describe the design and procedures of both experiments before describing the relevant data.

The participants for the laboratory experiment were 190 individuals (mostly students) from the

\footnotetext{
${ }^{12}$ Although unstated, the converse of all propositions are also true: a person with CARA preferences will never make dominated choices because with CARA the optimal choice within each bracket $i$ is independent of the distribution that is generated in the other brackets.

${ }^{13}$ The evidence on lottery choice behavior points at a decreasing degree of absolute risk aversion, for the average decisionmaker — see e.g. Holt and Laury (2002) for laboratory evidence. As in most related studies, this stylized result from Holt and Laury (2002) implicitly assumes fully narrow bracketing ( $\kappa=0$ in our notation) when inferring risk attitudes from lottery choice data. Dohmen et al (2005), in contrast, measure risk aversion also under the assumption of broad bracketing.
} 
subject pool of the ELSE laboratory at University College London. They participated in 15 sessions of sizes ranging between 7 and 18 particpants, in four different treatments. Each participant faced one treatment only, consisting of one particular variant of the $A / B / C / D$ choices of Example 1 . The wording was as given in the introduction.

In the first treatment, "Incentives-Small Scale", which was conducted in four sessions with $N=53$ participants in total, we used the payoffs of $A / B / C / D$ that were given in the introduction, and these payments were made for real. In a "Flat Fee-Small Scale" treatment (three sessions, $N=44$ ), paticipants made the same two choices between $A$ and $B$ and between $C$ and $D$, but only the show-up fee was paid, as explained below. In the third treatment, "Incentives-Small Scale-Broad Presentation" (four sessions, $N=45$ ), they made only one four-way decision, choosing between the distributions of the sum of earnings that would result from the four possible combinations of $A$ and $C, A$ and $D, B$ and $C$, and $B$ and $D$. I.e., in this treatment we imposed a broad view on the two decision tasks by adding up the payoffs from the two decisions. For example, the combination of $A$ and $D$ would be presented as "a $25 \%$ chance to gain $£ 2.40$ and a $75 \%$ chance to lose $£ 7.60$."14 Finally, in a "Flat Fee-Large Scale" treatment (three sessions, $N=48$ ), the participants made the two hypothetical choices of the second treatment, but we mutiplied all payoff numbers by a factor of 100 . Hence, they could make hypothetical gains and losses of up to $£ 1,000$ in this treatment.

On the first sheet of the experimental instructions, it was clarified that all random draws in the course of the experiment would be independent draws and outcomes would be determined by coin flips. All choices were made in a paper-and-pencil format, with only very few oral announcements that followed a fixed protocol for all treatments, and with the same experimenter present in all sessions. After the choices on Example 1, the experiments moved on to a second part. This second part is not analyzed in the paper, but the tasks are briefly summarized in Table 1 and described in detail in the appendix. The part II tasks differed between the 15 sessions, but the participants were not made aware of the contents of the part II before making their part I choices, so that the Example 1 choices cannot have been affected by the differences in the second part. ${ }^{15}$ The participants also

\footnotetext{
${ }^{14}$ In this treatment, the order of the four choice options was randomly changed between the participants. In the three treatments with two binary choices, we maintained the same order as in Tversky and Kahneman (1981).

${ }^{15}$ In the second part, we tried out different lottery-choice problems that also addressed narrow-bracketing issues.
} 
had to fill in a questionnaire and a sheet with five mathematical problems. Finally, the relevant random draws were made and the participants were paid in cash. The entire procedure (including payments) took about 40-50 minutes in each session.

An email was sent to the participants 24 hours before the session in which they participated, and made them aware that (i) they would receive a show-up fee of 222 , (ii) that they "may" make gains and losses relative to their show-up fee, and (iii) that overall, they would be "about equally likely to make gains as losses (on top of the £22)."16 Upon arrival at the laboratory, the participants learned whether the experiment used monetary incentives or not, i.e. whether the outcome amounts were added to/subtracted from their show-up fee. This procedure aims at minimizing possible effects of earnings differences between treatments with hypothetical and real payments, by ruling out both ex-ante differences and anticipated ex-post differences in average earnings. In those sessions where we used real monetary incentives, the second part of the experiment was designed such that the expected average of total earnings would indeed be at $£ 22 .{ }^{17}$ A further role of the 24-hour advance notice about the show-up fee was to make the losses more akin to real losses, as the participants may have "banked" the show-up fee. The amount $£ 22$ was not repeated on the day of the experiment before the subjects had made their Example 1 choices, and all gains and losses were presented using the words "gain" and "lose". Table 1 summarizes the sessions, including a brief description of the problems in part II of the experiment. In this description, "LC task" is short for a lottery choice task between a 50/50 lottery and a sure outcome and "CE task" is short for a task where the participants are asked to state their certainty equivalent for a given 50/50 lottery. "Example 2 " will be presented in the next subsection, and more detail on the design of part II can be found

These problems were used as a pre-test for the survey experiment, and potentially for further laboratory experiments. The initially announced payoff regime with respect to real versus hypothetical payments was always maintained in the second part.

${ }^{16}$ The email text, and complete instructions of all experiments, are available upon request.

${ }^{17}$ On average, the subjects received $£ 21.85$ in these sessions, with a standard deviation of $£ 7.70$. 
in the appendix.

\begin{tabular}{|c|c|c|c|}
\hline Treatment (part I) & \# of obs. & Sessions & Description of part II tasks \\
\hline Incentives-Small Scale & 53 & $1-4$ & $\begin{array}{l}\text { One of three LC tasks }(\mathrm{N}=33) \text {, or } \\
\text { two LC tasks similar to Example } 2(\mathrm{~N}=20)\end{array}$ \\
\hline Flat Fee-Small Scale & 44 & $5-7$ & $\begin{array}{l}\text { One of three LC tasks }(\mathrm{N}=35) \text {, or } \\
\text { two LC tasks similar to Example } 2(\mathrm{~N}=9)\end{array}$ \\
\hline Incentives-Small Scale-Broad Presentation & 45 & $8-11$ & $\begin{array}{l}\text { Two LC tasks analogous to Example } 2(\mathrm{~N}=21) \text {, or three } \\
\text { CE tasks for } 50 / 50 \text { between }\{-10,0\},\{-5,5\} \text { and }\{0,10\}(\mathrm{N}=24)\end{array}$ \\
\hline Flat Fee-Large Scale & 48 & $12-15$ & Two LC tasks analogous to Example $2(\mathrm{~N}=48)$ \\
\hline
\end{tabular}

The survey experiment used the survey tool of TESS (Time-Sharing Experiments in the Social Sciences), which regularly conducts questionnaire surveys with a stratified sample of American households, those on the Knowledge Networks panel. The panel members were recruited based on their telephone directory entries, and are all used to answering questions via special TV-connected terminals at their homes. For each new study, they are contacted by email. In the case of our questionnaire, a total of 1,910 panel members was contacted, of whom 1,292 fully completed the study. A further 30 respondents participated, but left at least one question unanswered. (We included their responses in the analysis, wherever possible.) Each participant was presented with one or several decision tasks, plus a short questionnaire that asked for information on mathematics education and gave the participants three mathematical problems to solve. The data set also contains information on each participant's personal background characteristics such as gender, employment status, income, and obtained level of education.

None of the lotteries was paid out, i.e. all choices were hypothetical. The amounts used in the decision tasks ranged from $-\$ 1550$ to $+\$ 2500$.

In addition to the binary lottery choices we report here, subjects were also asked separate questions in which they were asked to state certainty equivalents for 11 different lotteries. In Appendix 3 , we describe these experiments and the data, and discuss why we feel these certainty equivalence 
data are unreliable, as many participants cannot plausibly have understood the procedure.

Participants were randomly assigned to 10 different treatment groups, and each treatment contained a different set of one, two or six decision tasks (including lottery choices and certainty equivalent statements). Within each decision, the order in which the choice options appeared was random across participants. Table 2 summarizes the tasks in each of the 10 treatments. Further details on the tasks are given in the paragraphs following the table.

In each treatment, the participants' interfaces were programmed such that the participants had to read through all their decisions first, before they could make their choices. Importantly, the instructions that were presented to them on the first screen stated clearly that they should make their choices as if all of their outcomes were paid. Hence, it is unlikely that choices were made under a misunderstanding that only subsets of the decisions were relevant. Also, as in the 
laboratory experiment, the instructions made clear that all random draws were independent.

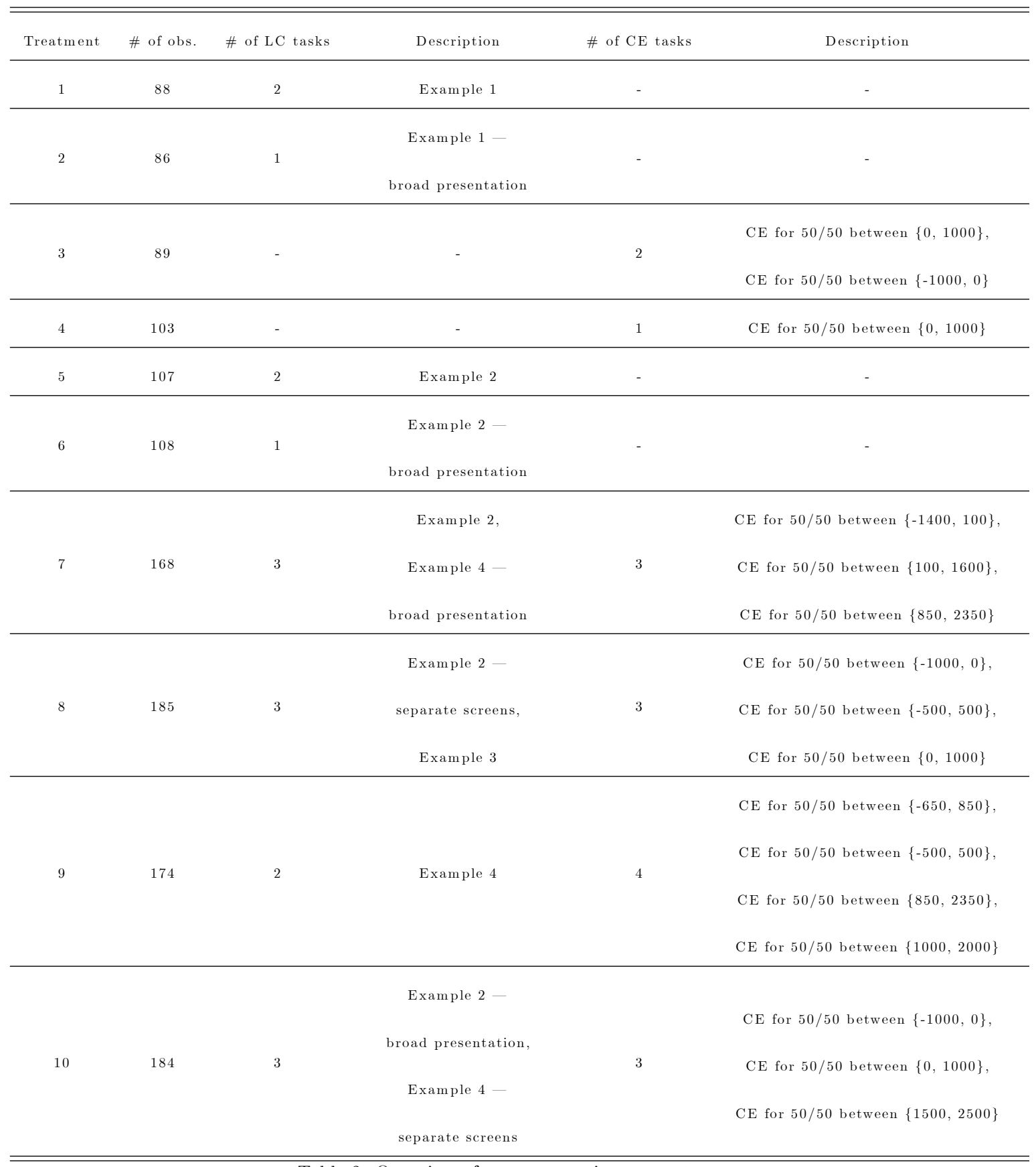

Table 2: Overview of survey experiment treatments

The lottery choice tasks are similar to those in Tversky and Kahneman's example, but with a 
slightly different wording. ${ }^{18}$ Our first set of decisions is parallel to the original example:

\section{Example 1:}

Decision 1: Choose between:

A. winning $\$ 240$

B. a $25 \%$ chance of winning $\$ 1000$ and a $75 \%$ chance of not winning or losing any money

Before answering, read the next decision.

Decision 2: Choose between:

C. losing $\$ 750$

D. a $75 \%$ chance of losing $\$ 1000$, and a $25 \%$ chance of not winning or losing any money

This replication was conducted in two treatments, once as descibed above (Treatment 1) and once in the broad four-way presentation (Treatment 2), where we explicitly offered the four combined choices $A C, A D, B C$ and $B D$, analogous to the third laboratory treatment. In both of these treatments, the participants made no other choices.

In all other treatments, we used only 50/50 gambles. The following are the new examples that we designed to generate dominance violations (the labels of choice options were changed from the instructions, for the sake of the exposition):

\section{Example 2:}

Decision 1: Choose between:

A. not winning or losing any money

B. a $50 \%$ chance of losing $\$ 500$ and a $50 \%$ chance of winning $\$ 600$

Before answering, read the next decision.

Decision 2: Choose between:

C. losing $\$ 500$

\footnotetext{
${ }^{18}$ Notice further the fact that we used USD amounts in the survey experiment, and GBP amounts in the laboratory experiment.
} 
D. a $50 \%$ chance of losing $\$ 1000$, and a $50 \%$ chance of not winning or losing any money

Example 2 was designed to bring loss aversion into play: if participants weigh losses heavier than gains, they will tend to choose $A$ over $B$, and if they are risk seeking in losses, they will tend to choose $D$ over $C$. Such a combination is dominated with an expected loss of $\$ 50$ relative to the reversed choices. This example, too, was conducted in isolation - i.e. with no other decisions for the participants - and presented as stated here (Treatment 5) and in isolation and presented in a broad-bracketing way (Treatment 6). In addition, the example was presented together with other decisions, in three different ways. In Treatment 7, the two decisions appeared on the same screen. In Treatment 8, they appeared on separate screens, with four other decisions appearing in between. This variation was included to detect potential effects of distractions caused by other choices. In Treatment 10, the example was presented as a broad-bracketed four-way choice, alongside with other decisions.

Example 3:

Decision 1: Choose between:

A. winning $\$ 1500$

B. a $50 \%$ chance of winning $\$ 1000$, and a $50 \%$ chance of winning $\$ 2100$

Before answering, read the next decision. [...]

Decision 2: Choose between:

C. losing $\$ 500$

D. a $50 \%$ chance of losing $\$ 1000$, and a $50 \%$ chance of not winning or losing any money

Similar to Example 1, this example uses possible risk aversion in gains and risk lovingness in losses. The choice of $A$ and $D$ is dominated with a loss of $\$ 50$ on average. The example was only conducted as stated here, in Treatment $8 .^{19}$ An important new feature of the example is that all possible combined outcomes involve positive amounts. Therefore, although a narrow evaluation of the second decision would consider negative payoffs, a broad-bracketing decisionmaker's choices can

\footnotetext{
${ }^{19}$ In treatment 8 , the second decision of this example is also the second decision of Example 2, so that within the data of Treatment 8 , the occurances of dominance violations are correlated between the two examples.
} 
only be influenced by preferences for lotteries above zero. In particular, under the assumption of broadly-bracketed choice, a decision for $D$ over $C$ would be evidence of risk-lovingness in gains. ${ }^{20}$

Example 4:

Decision 1: Choose between:

A. winning $\$ 850$

B. a $50 \%$ chance of winning $\$ 100$ and a $50 \%$ chance of winning $\$ 1600$

Before answering, read the second decision.

Decision 2: Choose between:

C. $\quad$ losing $\$ 650$

D. a $50 \%$ chance of losing $\$ 1550$, and a $50 \%$ chance of winning $\$ 100$

This final example uses a slightly more difficult spread between the payoffs and it involves some payoffs that are not multiples of $\$ 100$. As before, a decisionmaker who rejects the risk in the first decision but accepts it in the second decision $(A$ and $D)$ would violate dominance, here with an expected loss of $\$ 75$ relative to $B$ and $C$. An new feature is that these choices sacrifice expected value in the second decision, not in the first. This implies that for essentially all broad-bracketing risk averters the combined choice of $A$ and $C$ would be optimal: it generates the highest available expected value at the lowest available variance. Different from the other examples, the prediction for a broad-bracketed risk averter is therefore independent of the exact nature of her preferences. A further property of the example is that $A$ and $C$ would be predicted even for some narrow bracketers who have preferences like in Prospect Theory, with diminishing sensitivity for larger gains and losses, loss aversion, and risk aversion/risk lovingness in the gain/loss domains. This is because the risky choice $D$ involves a possible gain of $\$ 100$ so that a prospect-theoretic decisionmaker would only accept the gamble $D$ if the preference for risk in the loss domain is strong relative to the effect of

\footnotetext{
${ }^{20}$ This is true only if Example 3 is viewed separately from the third lottery choice decision in this treatment (Decision 1 in Example 2). Considering all three decisions, the treatment involves eight possible choice combinations, only one of which involves a possible loss as only one of its eight possible outcomes. Hence, the choice of $D$ in Example would still be indicative of a preference for risk with almost all payoffs being positive.
} 
loss aversion (which makes her averse to lotteries with payoffs on both sides of zero). In particular, the preference for risk in the loss domain needs to be slightly stronger than in the often-used parameterization of Tversky and Kahneman (1992) - see footnote 27. Under the assumption of narrow bracketing, the example therefore helps to discriminate between different plausible degrees of risk lovingness in the loss domain. The example was conducted in Treatments 7, 9, and 10, with differences between broad versus narrow presentation, and with and without other decisions appearing in between the two decisions.

\section{Experimental results}

\subsection{Data Summary}

Table 3 lists the frequencies of observing each of the four possible choice combinations in Example 1 , in the four different laboratory treatments. The results of each of the treatments' second parts are presented in the appendix. ${ }^{21}$

\begin{tabular}{|c|c|c|c|c|c|}
\hline Treatment & \# of obs. & $\mathrm{A}$ and $\mathrm{C}$ & $A$ and $\mathrm{D}$ & $\mathrm{B}$ and $\mathrm{C}$ & $B$ and $D$ \\
\hline Incentives, Small Scale & 53 & 0.21 & 0.28 & 0.11 & 0.40 \\
\hline Flat Fee, Small Scale & 44 & 0.16 & 0.34 & 0.09 & 0.41 \\
\hline Incentives, Small Scale, Broad Presentation & 45 & 0.11 & 0.00 & 0.38 & 0.51 \\
\hline Flat Fee, Large Scale & 48 & 0.15 & 0.54 & 0.08 & 0.23 \\
\hline
\end{tabular}

Table 3: Choice frequencies in Example 1 - different laboratory treatments.

There is little difference between the observed behavior in the first two treatments. No matter whether the outcomes were actually paid or not, about half of the respondents chose the sure option

\footnotetext{
${ }^{21}$ We do not analyze the part II results further, noting that they are subject to possible influence of the first parts and have few observations. The three lottery-choice tasks in the first two treatments were modelled after Redelmeier and Tversky's (1992) investment experiment. We abandoned further such tests when the data indicated that the low level of behavioral variation between the three conditions would require large sample sizes to observe any effects. Generally, the participants made much more risk-seeking choices in all part II tasks; in the task similar to Example 2, only few FOSD violations appeared (see Tables 7.1, 7.2, and 7.3).
} 
of $A$ over the lottery $B$, and slightly more than two thirds chose the uncertain loss of lottery $D$ over the sure loss in $C$. This confirms Prospect Theory's prediction of risk seeking behavior in the losses domain, although the evidence in the first decision is less clear. 28 percent and 34 percent of the two treatments' participants, respectively, chose the dominance-violating combination of the risk-averting $A$ and the risk-seeking $D$. The difference in these two frequencies is insignificant at any conventional level. A one-tailed Fisher exact test of the null hypothesis that the frequency of observing $A$ and $D$ is identical under both treatments rejects at $p=0.346$, and therefore finds no support for the alternative hypothesis that the frequency of dominance violations decreases if the decisions are paid for real. Similarly, testing for differences in the entire distribution of choices (not just in the frequency of $A$ and $D$ ), a two-tailed Pearson chi-square test rejects the null hypothesis of no difference at $p=0.866$. Overall, there is no statistically significant effect of actually paying the (small-scale) decisions in this data set.

A comparison of the Flat Fee-Small Scale treatment with the Flat Fee-Large Scale treatment shows that if the hypothetical payoff numbers are larger, more participants indicate dominated choices. The difference between the frequencies of observing $A$ and $D$ increases from 34 perccent to 54 percent, and this increase is significant between the two treatments $(p=0.042$, one-tailed Fisher exact test). ${ }^{22} 23$

Finally, a comparison between the Incentives-Small Scale treatment and the Incentives-Small Scale, Broad Presentation treatment suggests a strong effect of narrow bracketing. If the participants are forced to view the decision problem from a broad perspective, they make much fewer $A$ and $D$ choices ( $p<0.001$, one-tailed Fisher exact test), and also the overall distributions of choices are significantly different $(p<0.001$, two-tailed Pearson chi-square test). This clearly indicates that the subjects did not view the two decisions as a combined problem.

\footnotetext{
${ }^{22}$ But a Pearson chi-square test still supports the hypothesis of identical four-way distributions of choices, between the two treatments $(p=0.221$, two-tailed). In the light of the significant result of the Fisher exact test we attribute this failure to reject to the low numbers of observations.

${ }^{23}$ We note that the combination of the above comparisons does not reject the hypothesis that the difference in violation rates between our Incentives, Small Scale treatment and Tversky and Kahneman's (1981) experiment is largely driven by the larger payoff scale, not by the hypothetical/real payments difference. A positive confirmation of this hypothesis would require a very expensive experiment with large real payments.
} 
The following table summarizes the frequencies of the representative-survey responses in the four examples. The combination $A$ and $D$ is the dominated combination of choices in each of the four examples. ${ }^{24}$

\begin{tabular}{|c|c|c|c|c|c|c|}
\hline Treatment & \# of obs. & Description & $\mathrm{A}$ and $\mathrm{C}$ & $A$ and $D$ & $\mathrm{~B}$ and $\mathrm{C}$ & $B$ and $D$ \\
\hline 1 & 88 & Example 1 & 0.16 & 0.66 & 0.03 & 0.15 \\
\hline 2 & 86 & Example 1 - broad presentation & 0.22 & 0.06 & 0.24 & 0.48 \\
\hline 5 & 107 & Example 2 & 0.24 & 0.53 & 0.05 & 0.19 \\
\hline 6 & 108 & Example 2 - broad presentation & 0.09 & 0.38 & 0.12 & 0.41 \\
\hline \multirow{3}{*}{7} & & Example 2 & 0.22 & 0.53 & 0.05 & 0.20 \\
\hline & 171 & & & & & \\
\hline & & Example 4 - broad presentation & 0.72 & 0.04 & 0.13 & 0.12 \\
\hline \multirow[t]{2}{*}{8} & 186 & Example 2 - separate screens, & 0.26 & 0.44 & 0.07 & 0.23 \\
\hline & & Example 3 & 0.23 & 0.50 & 0.10 & 0.17 \\
\hline 9 & 179 & Example 4 & 0.35 & 0.36 & 0.16 & 0.13 \\
\hline \multirow[t]{2}{*}{10} & 184 & Example 2 - broad presentation, & 0.15 & 0.24 & 0.24 & 0.36 \\
\hline & & Example 4 - separate screens & 0.33 & 0.43 & 0.10 & 0.14 \\
\hline
\end{tabular}

Table 4: Results of lottery choices in different survey experiment treatments

The table shows that the frequencies of dominance violations is large when the examples are presented as two choices. Pooling across treatments where applicable, the average frequencies of $A$ and $D$ in Examples 1, 2, 3, and 4 are 0.66, 0.50, 0.50 and 0.40, respectively, out of four available combinations. $A$ and $D$ is the most frequently chosen choice combination in all four examples in the survey - note that this was not true in our small-scale laboratory treatments. Additional summaries need to be given for treatments 7,8 and 10 of the survey, where the participants made two binary choices and one four-way choice, which allows for a total of 16 choice combinations. Of these, dominance can be violated by any of 7 available choice combinations (all combinations that involve $A$ and $D$ in either example), and we find that the overall frequencies of choosing one of these 7 combinations are at $0.56,0.59$ and 0.55 , respectively.

\footnotetext{
${ }^{24}$ To interpret the reported frequencies of Treatment 8, recall that one decision is part of both Example 2 and Example 3.
} 
All of these observed violations are inconsistent with any model where rational agents with monotonic preferences make broad-bracketed choices. But the table also contains additional evidence against broad bracketing. The variation between narrow and broad presentation of Examples 1, 2 and 4 shows that the frequencies of dominated choice are significantly reduced in each of the broad-presentation tasks. In two cases the reduction in violation rates are very large (Examples 1 and 4, where they are reduced to 0.06 and 0.03), but in the case of Example 2, there remains a large proportion of respondents (0.29, summing across treatments) who choose the dominated $A$ and $D$ even when the choice is presented to them in a broad way. These puzzling choices cannot be explained even by narrow bracketing. ${ }^{25}$

Doubts about whether participants are broadly bracketing also arise from looking at the nature of preferences that a broad bracketer would have to have in order to make the observed choices, both within and across treatments. The design of Examples 3 and 4 is instrumental for this consideration: in Example 3, we observe that $67 \%$ of the participants choose $D$ over $C$, which is (as argued in Section 3) evidence of risk-lovingness in the domain of gains under the assumption of broad bracketing. However, the high frequencies of $A$ and $C$ in Example 4, particularly when the example is presented broadly (72\%), suggest the opposite - because the choice of $A$ and $D$ is predicted for a risk-averse broad bracketer in that example.

To make such a comparison of hypothetical underlying preferences more rigorous, it is convenient to look at treatments 7 and 10, which contain effectively the same set of 16 available choice combinations, only bracketed in different ways. One can ask whether any distribution of broadly bracketed preferences in the population would predict the choices in these two treatments. Obviously, no monotonic preferences would allow a dominated choice, so (considering the violation rates reported above) a distribution of broad monotonic preferences can at most generate $44 \%$ of the choices in these treatments. But because the available composite lotteries are identical and the allocation of participants into treatments was random, all choice shifts between the treatments are further evidence against broad bracketing. The largest possible proportion of choices that can be

\footnotetext{
${ }^{25}$ We have no good explanation for the high violation rate in this task. Perhaps (to give a couple of bad explanations) the fact that " $A$ and $D$ " has fewer nonzero outcomes led some participants to choose it, or " $B$ and $C$ " is unattractive due to the large-looming loss of $\$ 1000$ that may appear even larger when contrasted with the small gain of $\$ 100$.
} 
generated by a model with a stable distribution of broad-bracketing agents is therefore given by adding up the smaller of the two observed frequencies of all 9 undominated choice combinations. For brevity, we do not report the full distribution of choice combinations, but only report the added frequencies: at most $33 \%$ of all choices could be generated by broad bracketers with monotonic preferences, even if we allow for arbitrary heterogeneity in preference. Restricting preferences further in various ways yields some insights into the plausibility of different models. If we require that all agents have two-part CRRA preferences with a kink at $0,{ }^{26}$ and allow the resulting two parameters to vary arbitrarily across the population, we can explain up to $31 \%$ of the choices in treatments 7 and 10. In contrast, a distribution of CARA agents with different risk attitudes (a much less flexible model with one parameter per agent) could only explain up to $17 \%$. Restricting preferences to meet the most standard model of economic decisionmaking - expected utility over total earnings - would require near-risk-neutrality and could not explain virtually any of the participants' choices: this model would predict $B$ and $C$ in Example 2 and $A$ and $C$ in Example 4 but only $2 \%$ of the choices in treatment 7 follow this prediction. ${ }^{27}$

We also briefly summarize the evidence in Table 4 that speaks for or against models where agents have narrow brackets. As mentioned earlier, the surprisingly high frequency of $A$ and $D$ choices in the broad presentation of Example 2 represents a failure of all reasonable models, including those with narrow brackets. But at least in the binary choices, the results are consistent with narrowly-

\footnotetext{
${ }^{26}$ The utility function is $v(x)=\left\{\begin{array}{c}x^{1-\gamma} \text { for } x \geq 0 \\ -\delta\left(-x^{1-\gamma}\right) \text { for } x<0\end{array}\right\}$, as in the parametrization of Prospect Theory's value function in Kahneman and Tversky (1992).

${ }^{27}$ As a comparison, consider the prediction success Tversky and Kahneman's (1992) estimated version of Prospect Theory, with the functional form given in the previous footnote with parameters $\gamma=0.12$ and $\delta=2.25$. In treatment 7, the model would predict $A$ and $C$ in Example 4 and $A$ and $D$ in Example 2, wheras in treatment 10, it would predict $B$ and $C$ in Example 2 and $A$ and $C$ in Example 4. These predictions, too, are only partially successful, correctly predicting the choice combinations in $44 \%$ and $8 \%$ in the two treatments. In part, the poor performance in treatment 10 is driven by the strange behavior of the respondents in Example 2 under broad presentation. But furthermore, note that the above specification of Prospect Theory would not predict $D$ in the narrow presentation of Example 4, because the preference for risks in the negative domain is too small to offset the effect of the kink that discounts the high payoff of $\$ 100$ relative to the other payoffs. To predict $D$, the sensitivity parameter $\gamma$ would have to be at least 0.15 .
} 
bracketed preferences. In particular, it is straightforward to find prospect-theoretic preferences that have a very good fit: preferences that exhibit a sufficient degree of loss aversion and a sufficiently fast decrease in the sensitivity to gains and losses would make a prediction that coincides with the modal choice in each of the 13 binary choice problems contained in the survey - and a forteriori it would correctly predict the modal choice of $A$ and $D$ in all four examples.

The table also shows that the experimental variation of presenting the two tasks of Examples 2 and 4 on separate screens, and hence including other choices in between, yielded no strong effect. ${ }^{28}$ One can also ask more generally whether the inclusion of other tasks in a treatment appears to influence the choice frequencies. With the exception of the broadly presented Example 2 - which shows an effect towards fewer violations when other choices are included - there appears no such effect. In particular, all of the binary choices can be quite reliably predicted independent of other choices, but strongly dependent on the framing of the choice itself: in each of the four cases where a binary choice problem was framed as a risky choice with positive payoffs (the $A$-versus- $B$ problems in Examples 1, 3 and 4), at least $67 \%$ participants rejected the risk. In contrast, in each of the four cases where a binary choice was presented as a risky choice with negative payoffs (the $B$-versus- $C$ choices in Examples 1, 2 and 3), the risk was accepted by at least $67 \%$ of the participants.

Summing up, we find that broad-bracketing models can explain only a minority of choices, whereas at least the binary choices can be somewhat well organized by assuming narrow brackets, with risk aversion over gains and strong risk lovingness over loss. In the next subsection, we estimate simultaneously the preferences and brackets that fit the behavior best, to provide a fuller and more systematic statistical test of bracketing.

\footnotetext{
${ }^{28}$ The differences in the frequencies of $A$ and $D$ are statistically significant but small, and have opposite directions between the two examples. In Example 2, the frequency decreases from 0.53 to 0.44 when other choices are included ( $p=0.091$, two-tailed Fisher exact test) and in Example 4, the frequency increases from 0.36 to $0.43(p=0.026)$.
} 


\subsection{Simultaneous estimation of preferences and degree of bracketing}

\subsubsection{Estimates with pooled data}

We analyze the lottery-choice data from the survey experiment ${ }^{29}$ under the assumption that there exist two types of decisionmakers: One broad type who integrates her lottery-choice decisions into a joint decision problem (recall that each survey respondent has one, two or three lottery choices to make, depending on the treatment - see Table 2) and a narrow type who makes all decisions one-at-a-time. The broad type has frequency $\theta$ in the population, the narrow type $1-\theta$. Apart from their brackets, we assume that the decisionmakers have identical preferences represented by an expected utility function $v(\cdot)$. In order to generate a positive likelihood of observing any feasible choice vector, we assume logistic choice: The broad type calculates the expected utility $E[v(m)]$ from each available choice vector $m$, and probabilistically makes her choice according to

$$
\operatorname{Pr}(m \mid \lambda, \text { broad })=\frac{\exp (\lambda E[v(m)]}{\sum_{m^{\prime} \in M} \exp \left(\lambda E\left[v\left(m^{\prime}\right)\right]\right.} .
$$

The narrow type calculates the expected utitlity for each choice within each bracket $i$, and chooses each possible $m_{i}$ with probability

$$
\operatorname{Pr}\left(m_{i} \mid \lambda, \text { narrow }\right)=\frac{\exp \left(\lambda E\left[v\left(m_{i}\right)\right]\right.}{\sum_{m_{i}^{\prime} \in M_{i}} \exp \left(\lambda E\left[v\left(m_{i}^{\prime}\right)\right]\right.} .
$$

Hence, her choices in bracket $i$ are independent of the choices in other brackets. The overall likelihood of observing choice vector $m$ is

$$
\operatorname{Pr}(m \mid \theta, \lambda, u)=\theta \operatorname{Pr}(m \mid \lambda, \text { broad })+(1-\theta) \operatorname{Pr}(m \mid \lambda, \text { narrow })
$$

where the narrow type's likelihood of choosing the vector $m$ is calculated as the product $\operatorname{Pr}(m \mid \lambda$,narrow $)=$ $\Pi_{i} \operatorname{Pr}\left(m_{i} \mid \lambda\right.$, narrow $)$.

For the preferences $v$, we allow for a reference point $x_{0} \in[-1500,2500]$ and for a flexible hybrid CRRA-CARA utility function both above and below the reference point. ${ }^{30}$ The function is given

\footnotetext{
${ }^{29}$ The laboratory experiment is much less suitable for preference estimations, due to the small variation in choice sets.

${ }^{30}$ See Abdellaoui, Barrios and Wakker (2007) and Holt and Laury (2002) for related analyses with this hybrid function.
} 
by

$$
v(x)=\left\{\begin{array}{lc}
\frac{1-\exp \left(-r_{+}\left(x-x_{0}\right)^{1-\gamma_{+}}\right)}{r_{+}} & \text {if } x \geq x_{0} \\
-\frac{1-\exp \left(-r_{-}\left(x_{0}-x\right)^{1-\gamma}\right)}{r_{-}} & \text {otherwise }
\end{array}\right\},
$$

where $r_{+}, r_{-}, \gamma_{+}, \gamma_{-} \in(0,1)$, and $x_{0} \in[-1500,2500]$. The parameters $r_{+}$and $\gamma_{+}$govern the shape of the function for $x$-values above $x_{0}$, and $r_{-}$and $\gamma_{-}$for values below $x_{0}$. This separation into two separate domains introduces a kink at $x_{0}$ and makes $v$ very flexible in terms of allowing for changes in the degree of risk aversion. For $r_{+} \rightarrow 0$ or $r_{-} \rightarrow 0$, the respective parts above or below the reference points exhibit constant relative risk aversion, and for $\gamma_{+} \rightarrow 0$ or $\gamma_{-} \rightarrow 0$ they exhibit constant absolute risk aversion.

Simultaneously to estimating these five parameters of $v$, we estimate the noise parameter $\lambda$ and the proportion of broad types $\theta$. The following figure shows the ML-estimated $v$ function for the (standard) case that the reference point is restricted to lie at $x_{0}=0$.

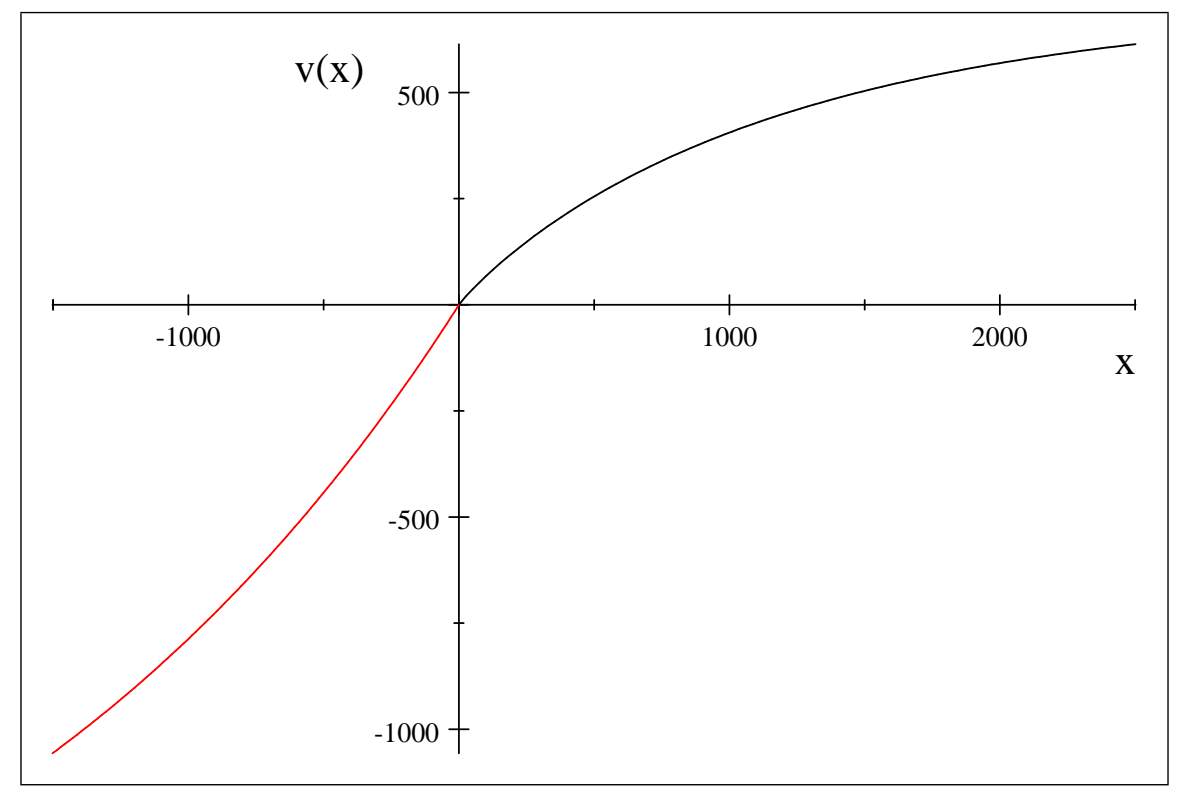

Figure 1: Estimated preferences $v$ under the restriction that $x_{0}=0$. Parameter estimates (and estimated standard deviations in parentheses $)$ are $\widehat{r}_{+, x_{0}=0}=0.0014(0.0004), \widehat{\gamma}_{+, x_{0}=0}=0.0740$

$$
\text { (0.0109), } \widehat{r}_{-, x_{0}=0}=0.0005 \text { (0.0001), and } \widehat{\gamma}_{+, x_{0}=0}=0.0000(0.0000) \text {. }
$$

The parameter estimates for $v$ are given in the caption of the figure. Simulateneously, the parameters $\theta$ and $\lambda$ are estimated to be at $\widehat{\theta}_{x_{0}=0}=0.1119$ (std. dev. 0.0491) and $\widehat{\lambda}_{x_{0}=0}=0.0133$ 
(0.0012), and the obtained log likelihood is $l l_{x_{0}=0}^{*}=-1926.4$.

The estimate $\widehat{\theta}_{x_{0}=0}=0.1119$ indicates the degree of broad bracketing: Only one out of nine choice vectors is estimated to be made by a broad-bracketing decisionmaker. The estimates of the preferences are very reminiscent of Prospect Theory's value function, with risk aversion around zero and in the positive domain, and a preference for risk in the negative domain. In part, this is because of the restrictions that we impose on the parameters: we require that $r_{+}, r_{-}, \gamma_{+}, \gamma_{-}$all lie in $(0,1)$, so that the function is necessarily concave above $x_{0}$ and convex below $x_{0}$. However, if we relax the restriction that $x_{0}=0$, then the degree of risk aversion is unrestricted at any given value in $[-1500,2500]$, and the function is generally more flexible. In particular, the function includes the special case of $v$ being a CARA function (for $x_{0}=-1500$ and $\gamma_{+} \rightarrow 0$ ), so that we can check whether the propositions of Section 2 can apply: there, we had concluded that the decisionmaker is in danger of making dominated decisions iff preferences do not exhibit contant absolute risk aversion. Figure 2 shows the estimated function $v$ for the estimation with general $x_{0}$.

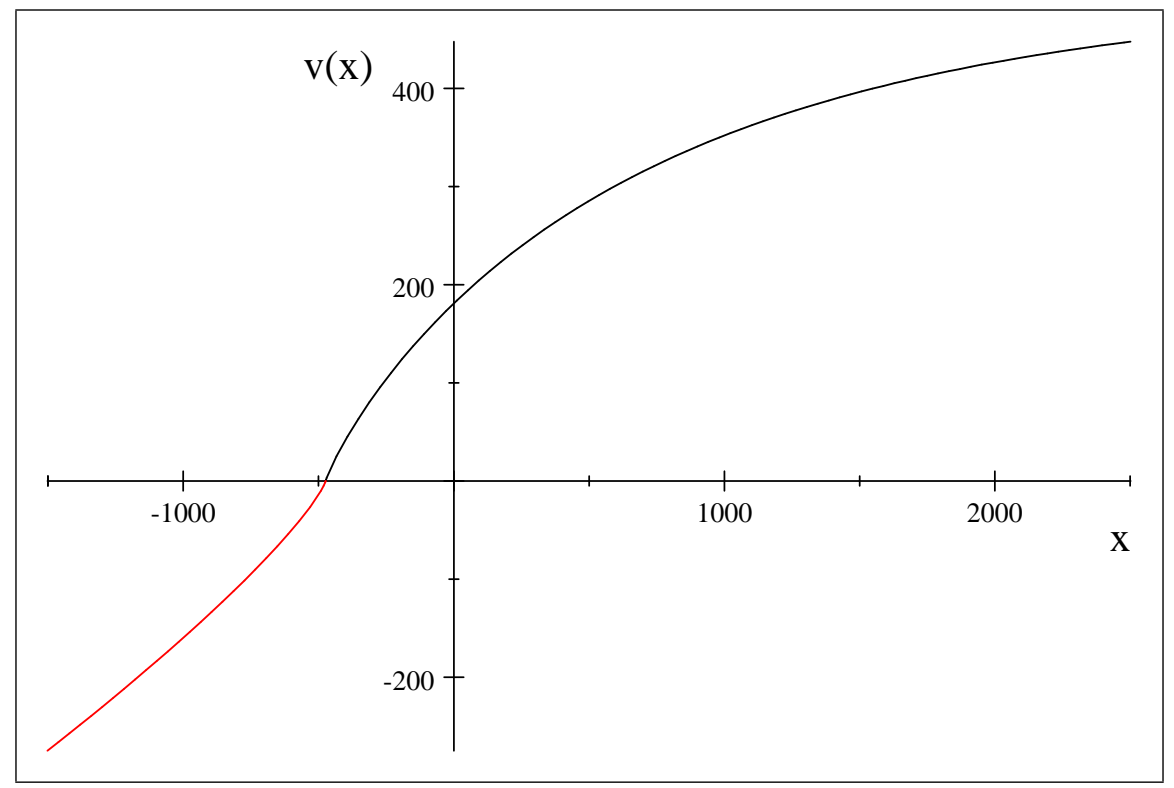

Figure 2: Estimated preferences of $v$ with unrestricted $x_{0}$. Parameter estimates (and estimated standard deviations in parentheses) are $\widehat{x}_{0}=-474.0(32.9), \widehat{r}_{+}=0.0020(0.0008), \widehat{\gamma}_{+}=0.1214$ (0.0835), $\widehat{r}_{-}=0.0000(0.0000)$ and $\widehat{\gamma}_{-}=0.1902(0.0617)$.

The figure shows that the estimated structural break lies at -474.0 . This is a surprising location 
that maximizes the fit of the model ${ }^{31}$ but on the whole does not change the general properties of $v$ with regard to relative changes in the degree of risk aversion. The decisionmaker is risk seeking for most gambles in the range below $x=0$, highly risk averse with respect to lotteries around $x=0$, and less risk averse for lotteries with only positive outcomes. The estimates of $\theta$ and $\lambda$ are $\widehat{\theta}=0.1666$ (std. dev. 0.0608) and $\widehat{\lambda}=0.0313$ (0.0157), and the obtained log likelihood is $l l^{*}=-1917.0$. Hence, in the most general formulation of the model we find that about one out of six choice vectors is estimated to be made by a broad bracketer.

We can now perform likelihood ratio tests to ask whether the estimated constellation of preferences and brackets is significantly different from its relevant special cases. First, we ask about CARA. The special case that $x_{0}=-1500$ and $\gamma_{+} \rightarrow 0$ yields a log likelihood of $l l_{C A R A}^{*}=-2131.8$, so that it is rejected at very high levels of significance. In fact, CARA's fit is not much better than that of a fully random model, which has a log likelihood of $l l_{\text {rand }}^{*}=-2158.5$. Invoking our theoretical results of Section 2, this failure of CARA to fit the data establishes that the decisionmakers are systematically subject to making dominated choices.

Next, we examine the importance of the degree of bracekting. First we restrict $\theta=0$, so that all decisionmakers are assumed to be narrow bracketers. This yields a log likelihood of $l l_{\theta=0}^{*}=-1920.4$. While this implies that the restriction is rejected at statistical significance of $p=0.009$, the $\log$ likelihood is still fairly close to that of the unrestricted model. In particular, the fit of the fully narrow model is hugely better than the fit of the fully broad model where $\theta=1$. This model yields $l l_{\theta=1}^{*}=-2120.0$. In other words, the hypothesis that all decisionmakers integrate their choices into a joint problem is strongly rejected, and is not much better than a fully random model of choice.

This test depends, of course, on the maintained assumptions about the preferences. In particular, we regard it as a strongly simplifying assumption that all agents are assumed to have the same preferences. But in the light of the data summary in the previous section, it seems impossible that allowing for heterogeneity would rescue the broad-bracketing model. There, we had found that even

\footnotetext{
${ }^{31}$ Notice that our experiment does not use any tasks that would provide "local" information at 0 or between -500 and 0 . This may help explain the location of the reference point. Notice also that to obtain the maximum likelihood, the convexity of $v$ at -500 is important because in our sample there are many choices rejecting a sure payoff of -500 . The kink generates this convexity.
} 
allowing for an arbitrary degree of heterogeneity, only small parts of the data can be accounted for within a broad-bracketing model. ${ }^{32}$

\subsubsection{Who has narrow brackets, and whose utilities differ from CARA?}

We now consider the background characteristics of the decisionmakers in the survey sample. The panel is designed to be representative of the general U.S. population and we have a variety of relevant characteristics in the data set. We separate the data set into pairs of subsamples according to a series of criteria (e.g. male/female respondents), and for each of these subsamples we reestimate the model of the previous subsection. Appendix 4 complements the analysis with a series of behavioral regressions, projecting the respondents' choices on their personal characteristics. This allows to check whether the differences between subgroups that we find in the model estimations are significantly reflected in the frequencies of accepting/rejecting certain lotteries and in the rate of violating dominance. The regression framework is also useful because for some of the explanatory variables it is important to include control variables. We will cross-refer to the regression results when discussing statistical significance of difference between the subsamples.

We use the following binary variables to create subsamples of the data, with numbers in parentheses indicating the numbers of individuals in each category who made at least one lottery choice (1109 in total): gender (532 male/577 female), age weakly below/strictly above the median age of 45 (548/561), "white, non-hispanic"/other as the self-reported racial/ethnic background $^{33}(815 / 294)$, household income weakly above/strictly below the median income ${ }^{34}(576 / 533)$, complete/incomplete set of correct answers to all three of our numerical questions (174/935), self-

\footnotetext{
${ }^{32}$ As another goodness-of-fit measure we counted the frequency with which a model predicts an observed lottery choice with the highest predicted likelihood, among the set of available choices. The best-fitting model among those with $\theta=0$ correctly predicts a total of $63.3 \%$ of all the lottery choices. In contrast, the best-fitting model with $\theta=1$ only has a rate of $47.6 \%$ correct predictions. Given that most choices are binary choices, this statistic further illustrates the weakness of the broad-bracketing model. A fully random model would correctly predict $44.6 \%$ of the choices. The value function of Kahneman and Tversky (1992), with their estimated parameters, correctly predicts $59.1 \%$ of the choices.

${ }^{33}$ In the full data set, the racial/ethnic background is coded as a categorical dummy with five categories.

${ }^{34}$ Household income is measured in 20 brackets. The median category is the bracket $[40,000 ; 49,999]$.
} 
reported attendance of a mathematics course in college (397/712), bachelor degree or higher/no bachelor degree (306/803).

Table 5 reports the parameter estimates and standard deviations for all seven pairs of subsamples, as well as the obtained log-likelihoods, under the restriction that the reference point lies at $x_{0}=0$. Figures 3-9 show the estimated preferences. For a comparison, the estimates for the full data set are repeated in the first column of the table, and the preferences are depicted in Figure 1.

The last row of Table 5 shows the percentage of participants in each subsample that made at least one $A$ and $D$ choice combination in one of the four examples. ${ }^{35}$

\begin{tabular}{|c|c|c|c|c|c|c|c|}
\hline & \multirow[t]{2}{*}{ All data } & \multicolumn{2}{|c|}{ Gender } & \multicolumn{2}{|c|}{ Age } & \multicolumn{2}{|c|}{ Racial/ethnic background } \\
\hline & & male & female & $<=45$ & $>45$ & white & non-white \\
\hline \multirow{2}{*}{$\theta$} & 0.1196 & 0.2120 & 0.0001 & 0.1565 & 0.0866 & 0.1272 & 0.0352 \\
\hline & $(0.0491)$ & $(0.0622)$ & $(0.0019)$ & $(0.0689)$ & $(0.0881)$ & $(0.0542)$ & $(0.1217)$ \\
\hline \multirow{2}{*}{$r_{+}$} & 0.0014 & 0.0010 & 0.0017 & 0.0012 & 0.0017 & 0.0015 & 0.0009 \\
\hline & $(0.0004)$ & $(0.0004)$ & $(0.0005)$ & $(0.0004)$ & $(0.0005)$ & $(0.0004)$ & $(0.0006)$ \\
\hline \multirow{2}{*}{$\gamma_{+}$} & 0.0740 & 0.0812 & 0.0617 & 0.0673 & 0.0796 & 0.0772 & 0.0544 \\
\hline & $(0.0109)$ & $(0.0149)$ & $(0.0159)$ & $(0.0134)$ & $(0.0146)$ & $(0.0122)$ & $(0.0149)$ \\
\hline \multirow{2}{*}{$r_{-}$} & 0.0005 & 0.0006 & 0.0004 & 0.0005 & 0.0006 & 0.0005 & 0.0005 \\
\hline & $(0.0001)$ & $(0.0001)$ & $(0.0001)$ & $(0.0001)$ & $(0.0000)$ & $(0.0001)$ & $(0.0001)$ \\
\hline \multirow{2}{*}{$\gamma_{-}$} & 0.0002 & 0.0000 & 0.0000 & 0.0000 & 0.0000 & 0.0000 & 0.0000 \\
\hline & $(0.0002)$ & $(0.0000)$ & $(0.0000)$ & $(0.0000)$ & $(0.0000)$ & $(0.0000)$ & $(0.0000)$ \\
\hline \multirow{2}{*}{$\lambda$} & 0.0133 & 0.0159 & 0.0113 & 0.0142 & 0.0125 & 0.0149 & 0.0101 \\
\hline & $(0.0012)$ & $(0.0019)$ & $(0.0013)$ & $(0.0018)$ & $(0.0024)$ & $(0.0014)$ & $(0.0025)$ \\
\hline obs. & 1109 & 532 & 577 & 548 & 561 & 815 & 294 \\
\hline$l l^{*}$ & -1926.4 & -913.2 & -1009.6 & -949.9 & -975.3 & -1381.2 & -537.5 \\
\hline$\% \mathrm{~A}$ and $\mathrm{D}$ & 50.1 & 49.3 & 50.8 & 46.7 & 53.3 & 53.3 & 41.7 \\
\hline
\end{tabular}

Table 5: Parameter estimates (st. dev. in parentheses) with $x_{0}=0$, data separated by variables.

\footnotetext{
${ }^{35}$ For ease of interpretation in the light of the estimation results, this percentage is calculated using only the narrow presentations of the four examples, not the broad presentation cases.
} 


\begin{tabular}{|c|c|c|c|c|c|c|c|c|}
\hline & \multicolumn{2}{|c|}{ Household income } & \multicolumn{2}{|c|}{ Math answers } & \multicolumn{2}{|c|}{ College math course } & \multicolumn{2}{|c|}{ Educational degree } \\
\hline & $>=40,000$ & $<40,000$ & 3 correct & $<=2$ correct & attended & not attended & bachelor & below bachelor \\
\hline \multirow{3}{*}{$\theta$} & 0.1539 & 0.0627 & 0.0928 & 0.1133 & 0.1615 & 0.0880 & 0.176 & 0.1082 \\
\hline & $(0.0681)$ & $(0.0577)$ & $(0.1334)$ & $(0.0471)$ & $(0.0697)$ & $(0.0666)$ & $(0.0705)$ & $(0.0449)$ \\
\hline & 0.0012 & 0.0017 & 0.0008 & 0.0016 & 0.0014 & 0.0014 & 0.0013 & 0.0015 \\
\hline & $(0.0003)$ & $(0.0007)$ & $(0.0004)$ & $(0.0004)$ & $(0.0006)$ & $(0.0005)$ & $(0.0005)$ & $(0.0005)$ \\
\hline \multirow{2}{*}{$\gamma_{+}$} & 0.0608 & 0.0876 & 0.0454 & 0.0815 & 0.0751 & 0.0718 & 0.0547 & 0.0834 \\
\hline & $(0.0116)$ & $(0.0201)$ & $(0.0150)$ & $(0.0127)$ & $(0.0160)$ & $(0.0143)$ & $(0.0158)$ & $(0.0139)$ \\
\hline \multirow{2}{*}{$r_{-}$} & 0.0004 & 0.0006 & 0.0003 & 0.0006 & 0.0005 & 0.0005 & 0.0005 & 0.0005 \\
\hline & $(0.0001)$ & $(0.0001)$ & $(0.0001)$ & $(0.0001)$ & $(0.0001)$ & $(0.0001)$ & $(0.0001)$ & $(0.0001)$ \\
\hline \multirow{2}{*}{$\gamma_{-}$} & 0.0000 & 0.0000 & 0.0000 & 0.0000 & 0.0000 & 0.0000 & 0.0000 & 0.0000 \\
\hline & $(0.0000)$ & $(0.0000)$ & $(0.0000)$ & $(0.0000)$ & $(0.0000)$ & $(0.0000)$ & $(0.0000)$ & $(0.0000)$ \\
\hline \multirow{2}{*}{$\lambda$} & 0.0163 & 0.0109 & 0.0201 & 0.0126 & 0.0159 & 0.0121 & 0.0170 & 0.0122 \\
\hline & $(0.0019)$ & $(0.0015)$ & $(0.0044)$ & $(0.0012)$ & $(0.0022)$ & $(0.0014)$ & $(0.0026)$ & $(0.0013$ \\
\hline obs. & 576 & 533 & 174 & 935 & 397 & 712 & 306 & 803 \\
\hline$l l^{*}$ & -967.9 & -951.7 & -282.1 & -1639.7 & -679.0 & -1245.3 & -516.7 & -1406.2 \\
\hline$\% \mathrm{~A}$ and $\mathrm{D}$ & 50.4 & 49.8 & 46.8 & 51.6 & 52.7 & 48.6 & 52.0 & 49.4 \\
\hline
\end{tabular}

Table 5 (ctd.): Parameter estimates (st. dev. in parentheses) with $x_{0}=0$, data separated by variables. 


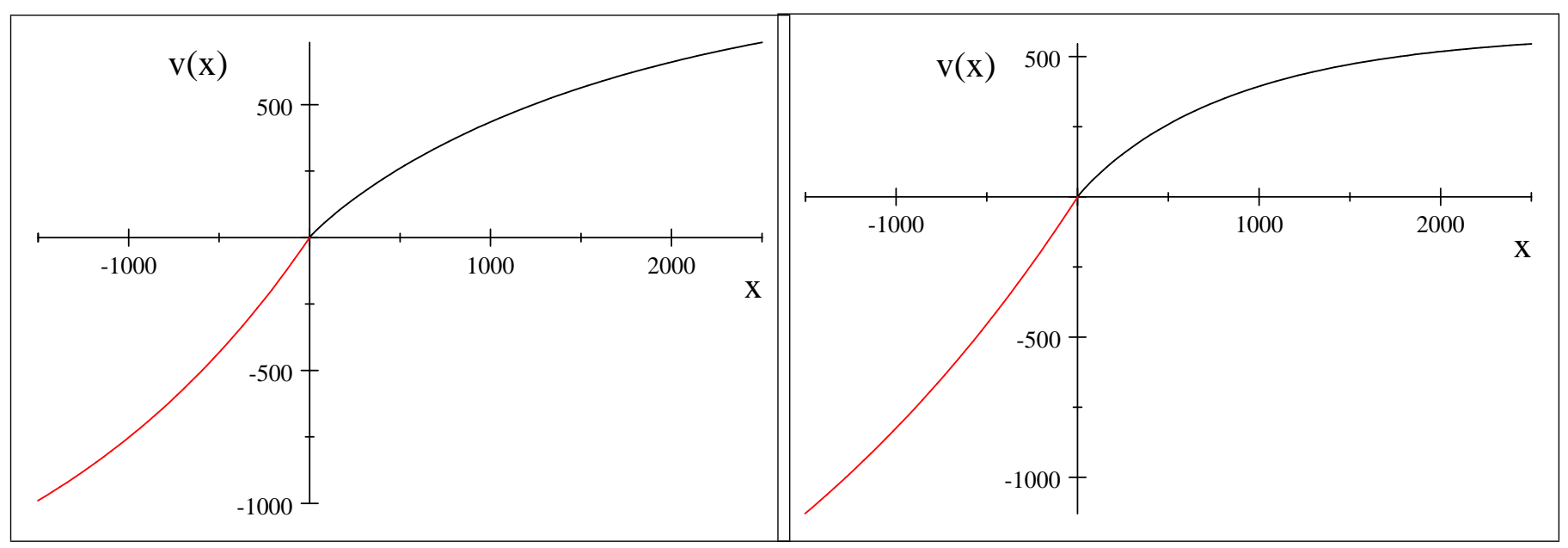

Figure 3a: Estimated $v(\cdot)$, male respondents

Figure 3b: Estimated $v(\cdot)$, female respondents

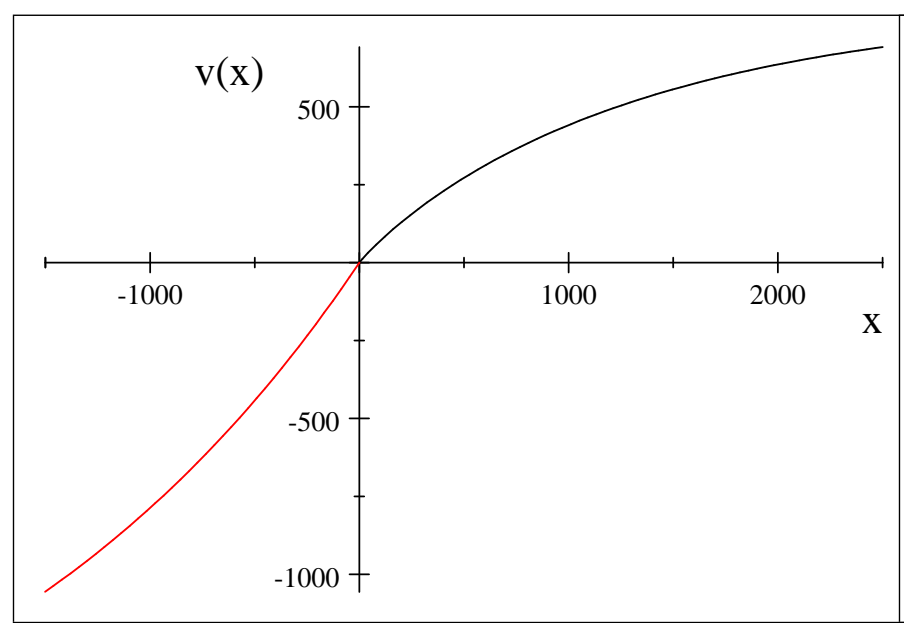

Figure 4a: Estimated $v(\cdot)$, respondents $\leq 45$ years

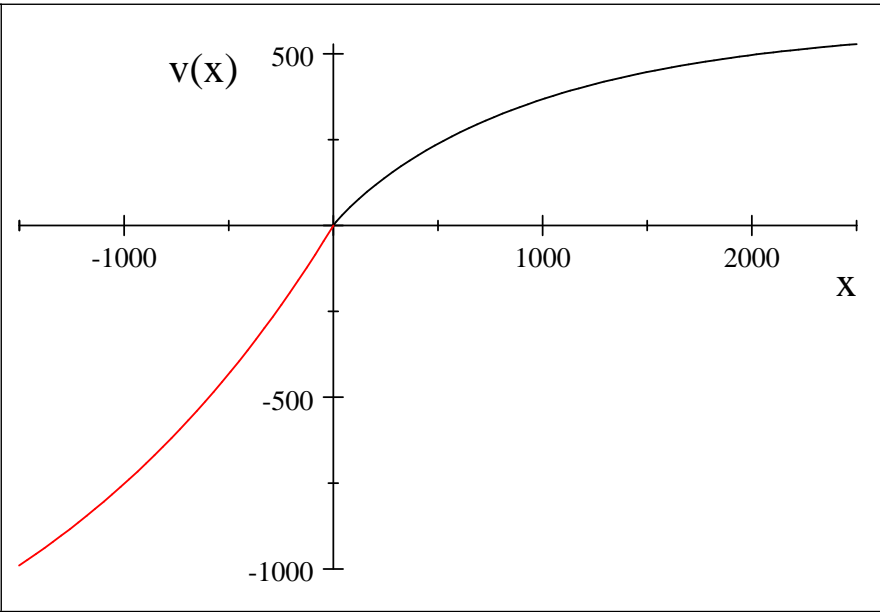

Figure $4 \mathrm{~b}$ : Estimated $v(\cdot)$, respondents $>45$ years 


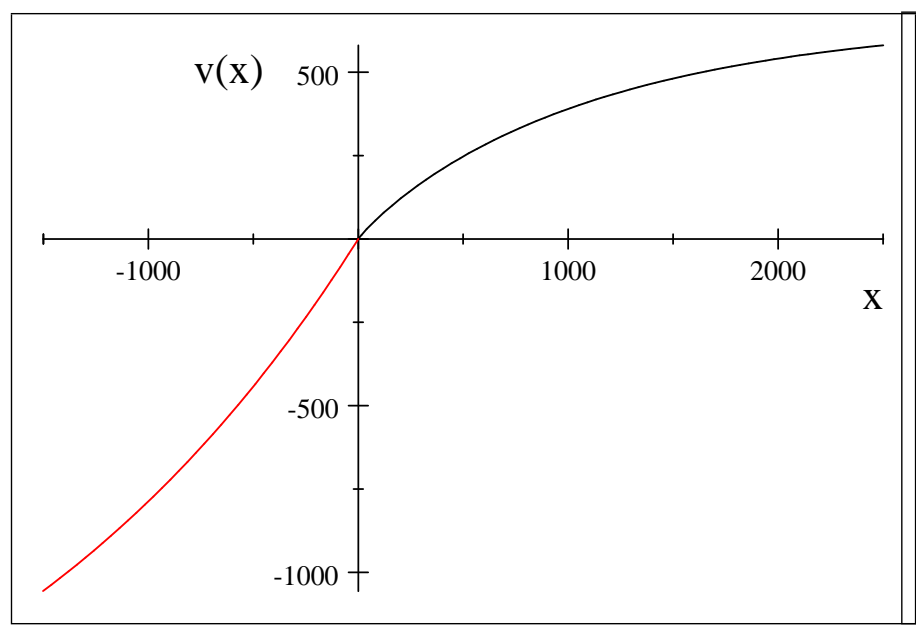

Figure 5a: Estimated $v(\cdot)$, white respondents

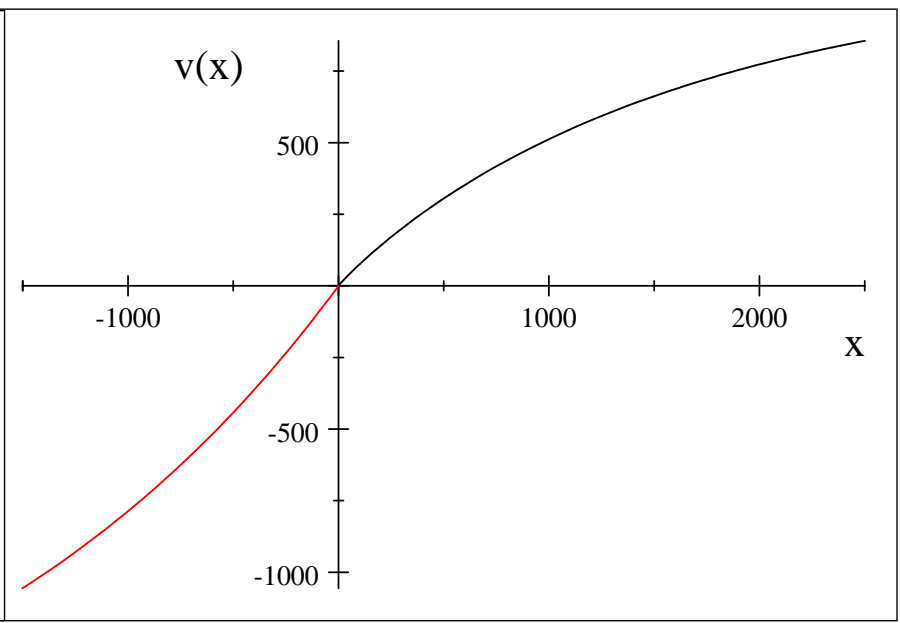

Figure 5b: Estimated $v(\cdot)$, nonwhite respondents

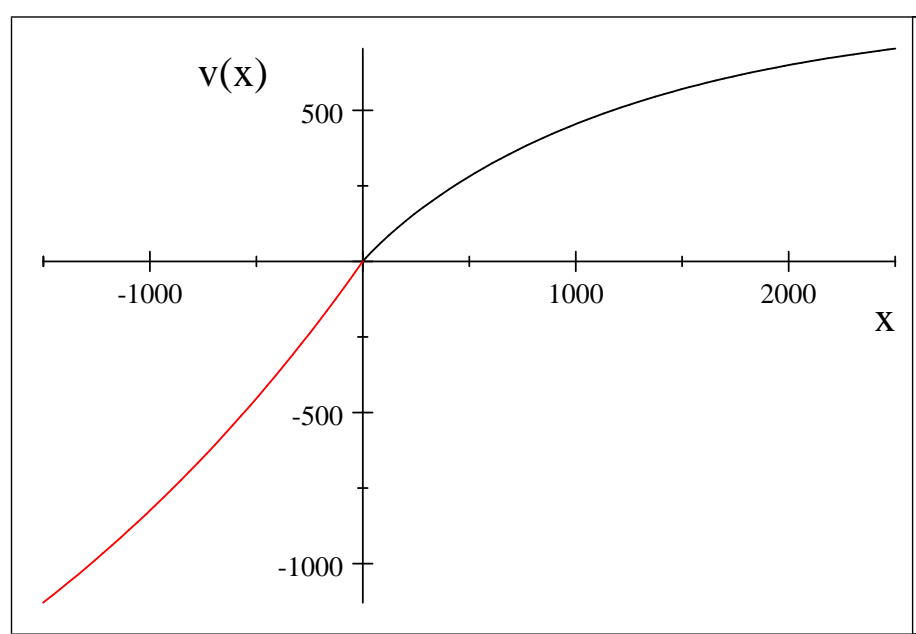

Figure 6a: Estimated $v(\cdot)$, higher-income respondents.

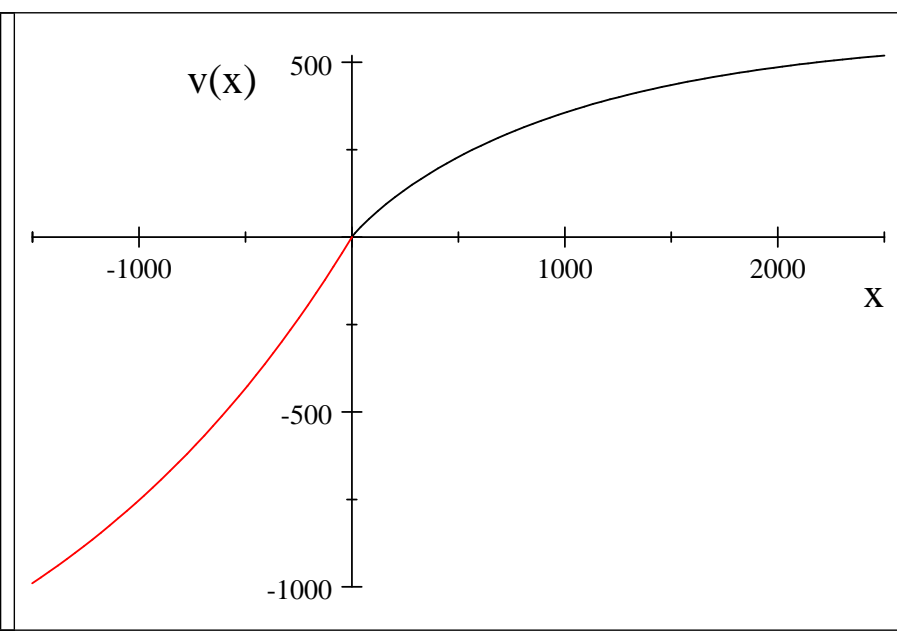

Figure 6b: Estimated $v(\cdot)$, lower-income respondents 


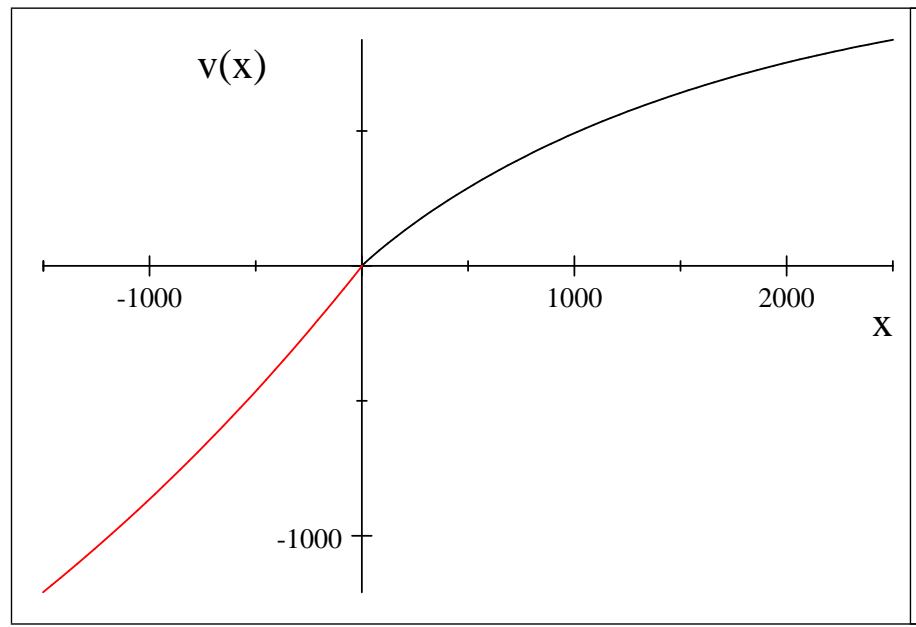

Figure 7a: Estimated $v(\cdot)$, math-skilled respondents

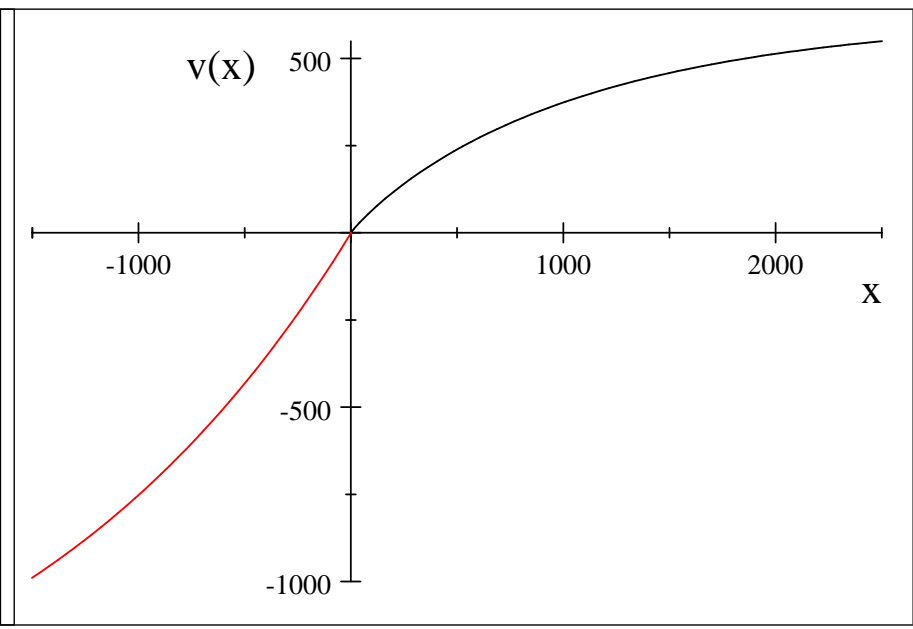

Figure 7b: Estimated $v(\cdot)$, less math-skilled respondents

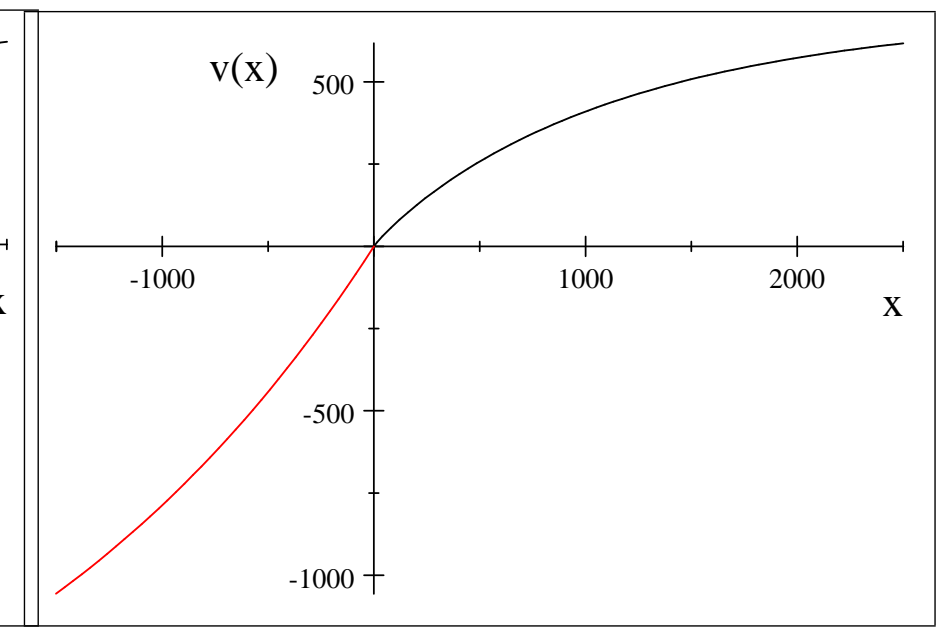
Figure 8b: Estimated $v(\cdot)$, non-math-educated resp.

Figure 8a: Estimated $v(\cdot)$, math-educated respondents 


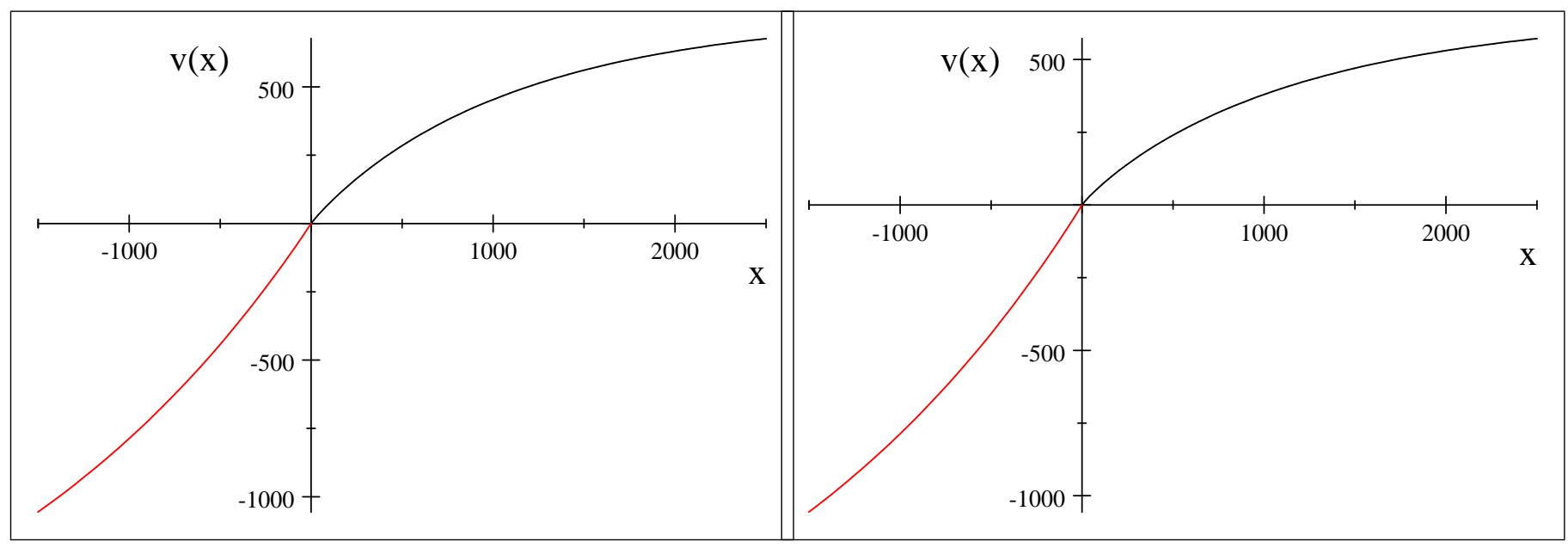

Figure 9a: Estimated $\mathrm{v}(\cdot)$, respondents with bachelor

Figure 9b: Estimated $\mathrm{v}(\cdot)$, respondents without bachelor

The estimation results and the figures show that the findings of the previous section are largely robust to the inclusion of background characteristics. All estimated preferences have essentially the same shape, and the estimated prevalence of broad bracketing $(\theta)$ lies below 0.22 in all subsamples. Likewise, almost all other parameter estimates are statistically indistinguishable between the pairs of subsamples. In a separate, unreported set of estimates, we also ran the analogous regressions without the restriction that $x_{0}=0$. The results are qualitatively identical to the ones presented here. In particular, all estimated preferences have the shape of Prospect Theory's value function although the unrestricted model would allow for much more flexibility - and with a single exception, all estimates of $\theta$ lie below $0.3 .^{36}$

But despite this robustness, there are some noticable differences between the subgroups. For example, male respondents have a significantly higher estimate of $\theta$ than female respondents. (In fact, women do not seem to integrate the choices at all, according to our estimates.) However, as indicated in the table's last row and confirmed in the the regressions of Appendix 4, this difference

\footnotetext{
${ }^{36}$ The exception is the subsample of non-white respondents, whose estimate of $\theta$ lies at 0.4 without the restriction. This difference under the more general model may also help to explain the behavioral differences between this subgroup and its comparison group, as described in the main text. Other differences between the more general estimation and the one with $x_{0}=0$ were minor, so that we decided to report the restricted estimates, which are easier to interpret.
} 
is not strong enough to generate significant differences in the violation rates - respondents of both genders have virtually identical frequencies of dominance violations. The reason may lie in the stronger convexity of men's utilities in the domain below zero, which leads them to have a slightly higher rate of accepting unbalanced risks.

Older respondents appear to be more loss averse, as the slope of their valuation function is steeper below zero. Their frequency of making a dominated choice is $14 \%$ higher than that of younger respondents, but this difference, too, is mostly insignificant in the regression analysis of Appendix 4.

A stronger and more significant effect appears between white and non-white respondents. Nonwhites are much more risk neutral towards lotteries around zero and in the domain above zero. This help them to avoid dominance violations, which is reflected in the fact that their violation rate is smaller than that of whites by $22 \%$. The regressions in Appendix 4 indicate that this is mostly due to a strong difference in the behavior of hispanics.

Further differences are in the more risk-neutral preferences for the groups of high-income and math-skilled respondents, relative to their comparison groups. But again, the differences do not carry over to a statistically reliable effect on behavior: dominance violation rates for high-income respondents are almost identical to those of low-income respondents and about $9 \%$ lower for the resondents who gave 3 correct mathematics answers, but none of this is significant in logistic regressions. This discussion partially confirms recent studies by Benjamin, Brown and Shapiro (2006), Frederick (2006) and Dohmen et al (2007) who find that risk preferences change systematically with measures of IQ or mathematics skills. ${ }^{37}$ Our evidence is consistent with these findings in that we also find more risk neutrality among the math-skilled respondents; but we do not find any robust effect on behavior, a discrepancy that may be due to the different pools of participants and/or to the different behavioral outcome variables. It is also worth pointing out that between the more and the less math-skilled respondents, we find no significant difference in $\theta$. Hence, it appears that it is not a question of numerical complexity that determines whether or not the decisionmakers

\footnotetext{
${ }^{37}$ Their measures are comparable to our three numerical questions - one of our questions is equivalent to a question that is used in Frederick (2006) and Benjamin, Brown and Shapiro (2006).
} 
integrate several choices into a joint choice problem. Even math-skilled respondents are susceptible to narrow bracketing, and therefore to making dominated choices.

\section{Conclusion}

The paper establishes that, under a very wide set of preferences, the failure to combine several decisions can lead a decisionmaker to make systematic mistakes in the form of dominated choices. But we have not explored at all whether such situations are likely to arise naturally, or whether economic actors out to make money (rather than social scientists out to make truth) will have the wish and the ability to induce narrowly-bracketing agents to make stochastically dominated choices. Indeed, the fact that the original example includes a choice among losses may mean that it would be hard for "dominance-entrepreneurs" to readily introduce such choice combinations, since presumably very few "clients" would volunteer to choose among losses. In this light, our results showing that neither the inclusion of an all-loss choice nor risk-lovingness are needed to induce dominance violations may add reason to believe that such violations might still be prevalent.

Against all this, we note that the frequency of dominated choices is not likely to be of special interest for welfare analysis. While inducing stochastic dominance is a way to establish that mistakes due to narrow bracketing exist independent of what preferences prevail (in the economy or in economic theory), the prevalence of such choices does not tell us how much utility the decisionmakers forgo. A complete welfare analysis of the losses due to narrow bracketing would measure the utility loss occuring with the preferences that people seem to have.

A final methodological note concerns the question of how to devise empirical estimates of risk preferences. Narrow bracketing implies that empirical estimates of risk attitudes will vary widely with the assumptions about the scope of the decision problem that the agents face, and how well those assumptions match the way agents themselves isolate choices in their minds. The currently prevalent approach of researchers reporting estimates that can be varied by several orders of magnitudes by dint of imposing varied assumptions about the scope of decisionmakers' choices needs a substitute. Our statistical analysis demonstrates that it may be possible to include a simultaneous estimation of the agents' degrees of bracketing so as to add more discipline to the measurement of 
risk attitudes.

\section{Appendix 1: Proofs}

Proof of Proposition 1: This is shown by a simple construction of two decision problems. Suppose w.l.o.g. that $L$ has the larger $N C E$, i.e. $N C E_{L}-N C E_{\widetilde{L}} \geq \delta$. Then the agent is more risk averse with respect to lottery $\widetilde{L}$, so to make the her indifferent between accepting $\widetilde{L}$ and a sure payment, a relatively smaller sure payment suffices. Offer her the following pair of choices, for small $\epsilon>0$ :

"Choose between lottery $\widetilde{L}$ and a sure payment of $C E_{\widetilde{L}}+\epsilon$ ".

"Choose between lottery $L$ and a sure payment of $C E_{L}-\epsilon$ ".

She will take the sure payment in the first choice, and the lottery in the second. In sum, she will own the joint lottery given by $C E_{\widetilde{L}}+\epsilon+L$. But she could have made the reverse choices, which would have resulted in the joint lottery $C E_{L}-\epsilon+\widetilde{L}$.

The lotteries' expected values satisfy $\mu_{\widetilde{L}}=\mu_{L}+\triangle x$. Using this together with $\widetilde{L}=L+\triangle x$ and $N C E_{L}-N C E_{\widetilde{L}} \geq \delta$, it follows that $C E_{L}-\epsilon+\widetilde{L}$ is identical to $C E_{\widetilde{L}}+\epsilon+L$ plus an sure payment of $\delta-2 \epsilon$. Hence, a dominance of size $\delta$ can be approached.

Proof of Proposition 2: Let $C A R A^{*}(\cdot)$ denote the CARA function that is closest to $v$ (or the limiting function if the minimum does not exist), i.e. reaches the distance $K$ at a " $K$-distance value" $x \in[\underline{x}, \bar{x}]{ }^{38}$ The proof proceeds in four steps and one lemma. The first three steps show the result for the case that $C A R A^{*}$ is concave, and step 4 covers the case that $C A R A^{*}$ is convex. Several additional notations will be used repeatedly in the proof: For a given binary lottery $L$ with

\footnotetext{
${ }^{38}$ Since $K$ is defined as an infimum over an open set of parameters $(\beta, \alpha, r) \in \mathbb{R}^{3}$ it may be that $K$ can only be approached but not reached with equality at some or all $K$-distance values - this may occur for one or more of the CARA parameters growing to infinity. To cover this case, the precise definition of a " $K$-distance value" is a value such that for all $\epsilon>0$ the horizontal distance lies within an $\epsilon$-neighborhood of $K$ for a sequence of CARA functions that converges to the limiting function. In the following, we will deal with the case that $K$ can be reached with equality at all $K$-distance points, but all statements and arguments extend to the case where $K$ can only be approached.
} 
possible outcomes $x_{L}^{\prime}$ and $x_{L}^{\prime \prime}$ and expected value $\mu_{L} \in\left(x_{L}^{\prime}, x_{L}^{\prime \prime}\right)$, let $L(\cdot)$ denote the function that describes the straight line connecting $\left(x_{L}^{\prime}, v\left(x_{L}^{\prime}\right)\right)$ and $\left(x_{L}^{\prime \prime}, v\left(x_{L}^{\prime \prime}\right)\right)$ (the "lottery line"). Let $C_{L}(\cdot)$ be the function describing a straight line through $\left(x_{L}^{\prime}, C A R A^{*}\left(x_{L}^{\prime}\right)\right)$ and $\left(x_{L}^{\prime \prime}, C A R A^{*}\left(x_{L}^{\prime \prime}\right)\right)$. Similarly, let $H_{L}(\cdot)$ describe the straight line through $\left(x_{L}^{\prime}, C A R A^{*}\left(x_{L}^{\prime}-K\right)\right)$ and $\left(x_{L}^{\prime \prime}, C A R A^{*}\left(x_{L}^{\prime \prime}+K\right)\right)$, and let $J_{L}(\cdot)$ describe the straight line through $\left(x_{L}^{\prime}, C A R A^{*}\left(x_{L}^{\prime}+K\right)\right)$ and $\left(x_{L}^{\prime \prime}, C A R A^{*}\left(x_{L}^{\prime \prime}-K\right)\right)$. The two lines $C_{L}$ and $H_{L}$ intersect at a point denoted by $x_{H_{L}}^{*}$, and the two lines $C_{L}$ and $J_{L}$ intersect at a point denoted by $x_{J_{L}}^{*}$. The lemma stated after the proof contains some properties of $x_{H_{L}}^{*}$ and $x_{J_{L}}^{*}$, as well as other properties of CARA functions.

From here on, we define net certainty equivalents with respect to the functions that represent underlying preferences. For example, for utility function $v$ and binary lottery $L$, the net certainty equivalent $N C E_{L}^{v}$ is defined as $C E_{L}^{v}-\mu_{L}$, where $C E_{L}^{v}$ satisfies the indifference condition $v\left(C E_{L}^{v}\right)=$ $\operatorname{Pr}\left(x^{\prime}\right) v\left(x^{\prime}\right)+\left(1-\operatorname{Pr}\left(x^{\prime}\right)\right) v\left(x^{\prime \prime}\right)$. Finally, for any given point $(x, v(x))$ on the graph of $v$ and for any given CARA function $\widetilde{C A R A}(\cdot)$, we describe the horizontal distance between $(x, v(x))$ and $\widetilde{C A R A}$ by the function $\eta(x, v, \widetilde{C A R A})=x-\widetilde{C A R A}^{-1}(v(x))$. Under the assumptions of the proposition, it holds that $\left|\eta\left(x, v, C A R A^{*}\right)\right| \leq K$ for all $x \in[\underline{x}, \bar{x}]$.

Step 1: Existence of four $K$-distance points on the graph of $v$ : Assume that $v$ has a horizontal distance of $K$ from the CARA family on $[\underline{x}, \bar{x}]$. Then $[\underline{x}, \bar{x}]$ contains (at least) four distinct $x$-values $\left\{x_{1}, x_{2}, x_{3}, x_{4}\right\}$ with $\left|\eta\left(x_{i}, v, C A R A^{*}\right)\right|=K$ for $i=1 \ldots 4$ and with three sign changes of $\left\{\eta\left(x_{i}, v, C A R A^{*}\right)\right\}_{i=1}^{4}$ from one value to the next: there exist $x_{1}<x_{2}<x_{3}<x_{4}$ such that either $\left[\eta\left(x_{1}, v, C A R A^{*}\right)=-K, \eta\left(x_{2}, v, C A R A^{*}\right)=K, \eta\left(x_{3}, v, C A R A^{*}\right)=-K\right.$ and $\eta\left(x_{4}, v, C A R A^{*}\right)=$ $K]$ or $\left[\eta\left(x_{1}, v, C A R A^{*}\right)=K, \eta\left(x_{2}, v, C A R A^{*}\right)=-K, \eta\left(x_{3}, v, C A R A^{*}\right)=K\right.$ and $\eta\left(x_{4}, v, C A R A^{*}\right)=$ $-K]$.

This statement holds because if there are fewer sign changes between $K$-distance values then $C A R A^{*}$ cannot be the closest CARA function but there exists a CARA function $C A R A_{\epsilon}^{\prime \prime}$ with the property that $\left|\eta\left(x, v, C A R A_{\epsilon}^{\prime \prime}\right)\right|<K-\iota$ for all $x \in[\underline{x}, \bar{x}]$ and some $\iota>0$. Towards a contradiction, assume that there are no more than two sign changes of $\eta\left(\cdot, v, C A R A^{*}\right)$ between $K$-distance values in $[\underline{x}, \bar{x}]$. With no more than two such sign changes, we can assume without loss of generality that 


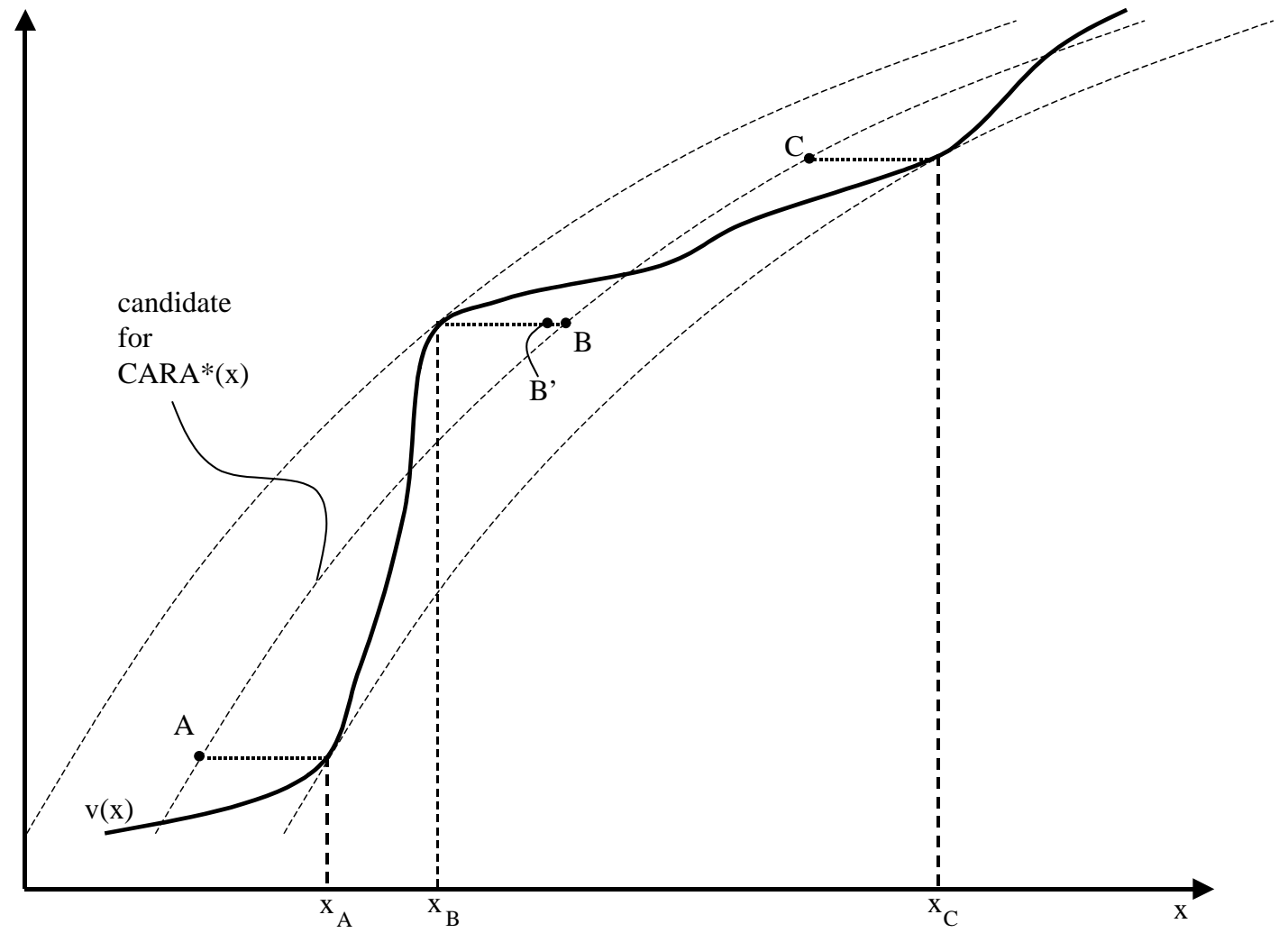

Figure 6.1: Three K-distance values 
the sign of $\eta\left(\cdot, v, C A R A^{*}\right)$ does not change from negative to positive and back to negative within the set of $K$-distance values - so we have a sequence like in Figure 6.1. ${ }^{39}$ We will discuss several cases, with the main argument given for Case 1 . For each case, denote by $X_{B}$ the (possibly empty) set of $K$-distance values that lie to the left of $C A R A^{*}$ so that $X_{B}=\left\{x \in[\underline{x}, \bar{x}] \mid \eta\left(x, v, C A R A^{*}\right)=-K\right\}$. If $X_{B}$ is nonempty, let $X_{A}$ and $X_{C}$ be the two (possibly empty) sets of $K$-distance values below $X_{B}$ and above $X_{B}$, respectively: $X_{A}=\left\{x \in[\underline{x}, \bar{x}] \mid x<X_{B}\right.$ and $\left.\eta\left(x, v, C A R A^{*}\right)=K\right\}$ and $X_{C}=\left\{x \in[\underline{x}, \bar{x}] \mid x>X_{B}\right.$ and $\left.\eta\left(x, v, C A R A^{*}\right)=K\right\}$. Then all $K$-distance values lie in the set $X_{A} \cup X_{B} \cup X_{C}$. If $X_{A}$ and $X_{C}$ are nonempty, select $x_{A}$ to be the maximum of $X_{A}$ and $x_{C}$ to be the minimum of $X_{C}$.

Case 1: The sets $X_{A}, X_{B}, X_{C}$ are all nonempty and $X_{B}$ is a singleton with a unique element $x_{B}$ : this case is depicted in Figure 6.1. (In the figure $X_{A}$ and $X_{C}$ are also singletons, but the arguments below cover the more general case of non-singleton sets.) By assumption, the minimum-distance CARA function is $C A R A^{*}$, depicted as the middle dashed line through points $A, B, C$. Consider the point $B^{\prime}$, with coordinates $\left(x_{B}+K-\epsilon, v\left(x_{B}\right)\right)$ for some small $\epsilon>0$. By the lemma, property (i), there exists a CARA function $C A R A_{\epsilon}^{\prime}$ that connects the three points $A, B^{\prime}, C$.

Consider the following two-step manipulation of CARA functions: first, replace $C A R A^{*}$ by $C A R A_{\epsilon}^{\prime}$; second, replace $C A R A_{\epsilon}^{\prime}$ by the function $C A R A_{\epsilon}^{\prime \prime}: x \rightarrow C A R A_{\epsilon}^{\prime}(x-\epsilon / 2)$ - i.e. shift $C A R A_{\epsilon}^{\prime}$ horizontally to the right by $\epsilon / 2$. For sufficiently small $\epsilon$, this manipulation will always be feasible and will result in a smaller horizontal distance to $v$ : let $\widetilde{X}_{\epsilon}=\left\{x \in[\underline{x}, \bar{x}]|| \eta\left(x, v, C A R A_{\epsilon}^{\prime \prime}\right) \mid>K-\epsilon / 4\right\}$ be the set of $x$-values that have a horizontal distance that is strictly larger than $K-\epsilon / 4$ if $C A R A^{*}$ is replaced by $C A R A_{\epsilon}^{\prime \prime}$. We will show that this set is empty for sufficiently small $\epsilon$, by checking all possible $x \in[\underline{x}, \bar{x}]$.

Checking $x \in X_{A} \cup X_{B} \cup X_{C}$ : for $x=x_{B}$ the two-step manipulation yields $\left|\eta\left(x, v, C A R A_{\epsilon}^{\prime \prime}\right)\right|=$ $\left|\eta\left(x, v, C A R A_{\epsilon}^{\prime}\right)\right|+\epsilon / 2=\left|\eta\left(x, v, C A R A^{*}\right)\right|-\epsilon / 2$ by construction of $C A R A_{\epsilon}^{\prime}$ and $C A R A_{\epsilon}^{\prime \prime}$. For $x \in$ $\left\{x_{A}, x_{C}\right\}$, the first manipulation from $C A R A^{*}$ to $C A R A_{\epsilon}^{\prime}$ does not change the horizontal distance

\footnotetext{
${ }^{39}$ This is equivalent to assuming that if the sign changes twice, then it is from positive to negative to positive, like in Figure 6.1. If the sequence of sign switches is from negative to positive to negative, then construct $X_{A}, X_{B}$, $X_{C}$ analogously, starting with the definition of $X_{B}=\left\{x \in[\underline{x}, \bar{x}] \mid \eta\left(x, v, C A R A^{*}\right)=K\right\}$. All ensuing argument are analogous.
} 
$|\eta|$, and the second manipulation reduces it by $\epsilon / 2$ so that $\left|\eta\left(x, v, C A R A_{\epsilon}^{\prime \prime}\right)\right|=\left|\eta\left(x, v, C A R A^{*}\right)\right|-$ $\epsilon / 2$. For $x \in X_{A} \cup X_{C} \backslash\left\{x_{A}, x_{C}\right\}$, i.e. for $K$-distance values that lie below $x_{A}$ and above $x_{C}$, the initial manipulation yields a strict reduction in $|\eta|$ for sufficiently small $\epsilon$ because $C A R A_{\epsilon}^{\prime}$ is more concave than $C A R A^{*}$, and the second manipulation yields a further reduction by $\epsilon / 2$. Hence, $\left|\eta\left(x, v, C A R A_{\epsilon}^{\prime \prime}\right)\right|>K-\epsilon / 4$ cannot hold at any initial $K$-distance value.

Checking $x \notin X_{A} \cup X_{B} \cup X_{C}$ : first observe that only in the vicinity of the initial $K$-distance values in $X_{A} \cup X_{B} \cup X_{C}$ can $v$ 's distance from $C A R A^{*}$ be arbitrarily close to $K$. Since $C A R A^{*}$ and $C A R A_{\epsilon}^{\prime \prime}$ converge for $\epsilon \rightarrow 0$, this observation implies that $\tilde{X}_{\epsilon}$ converges to $X_{A} \cup X_{B} \cup X_{C}$ as $\epsilon \rightarrow 0$. Hence, any sequence of pairs $\left(\epsilon, \widetilde{x}_{\epsilon}\right)$ such that $\epsilon \rightarrow 0$ and $\widetilde{x}_{\epsilon} \in \widetilde{X}_{\epsilon}$ has the property that $\widetilde{x}_{\epsilon}$ lies arbitrarily close to $X_{A} \cup X_{B} \cup X_{C}$ for sufficiently small $\epsilon$. But $\widetilde{x}_{\epsilon}$ cannot lie to the left of $x_{A}$ or to the right of $x_{C}$, by the same argument that we gave above for $x \in X_{A} \cup X_{C} \backslash\left\{x_{A}, x_{C}\right\}$ : for any such values the two manipulations will yield a reduction in horizontal distance by strictly more than $\epsilon / 2$ so that $\left|\eta\left(\widetilde{x}_{\epsilon}, v, C A R A_{\epsilon}^{\prime \prime}\right)\right|>K-\epsilon / 4$ can hold only if $\widetilde{x}_{\epsilon} \in\left(x_{A}, x_{C}\right)$. It follows that $\widetilde{x}_{\epsilon}$ must become arbitrarily close to the set $\left\{x_{A}, x_{B}, x_{C}\right\}$ so that there exists a subsequence of $\widetilde{x}_{\epsilon}$, $\widetilde{\widetilde{x}}_{\epsilon}$, that converges to $x_{A}, x_{B}$ or $x_{C}$.

Could $\widetilde{\widetilde{x}}_{\epsilon}$ converge to $x_{A}$ ? $\widetilde{\widetilde{x}}_{\epsilon}$ needs to satisfy $\left|\eta\left(\widetilde{\widetilde{x}}_{\epsilon}, v, C A R A_{\epsilon}^{\prime \prime}\right)\right|>K-\epsilon / 4$, which implies $\left|\eta\left(\widetilde{\widetilde{x}}_{\epsilon}, v, C A R A_{\epsilon}^{\prime}\right)\right|>K+\epsilon / 4$, since $C A R A_{\epsilon}^{\prime \prime}$ results from a right shift of $C A R A_{\epsilon}^{\prime}$ by $\epsilon / 2$. But this means that as $C A R A^{*}$ is replaced by $C A R A_{\epsilon}^{\prime}$ in the first manipulation, the horizontal distance $\eta(x, v, \cdot)$ increases at $x=\widetilde{\widetilde{x}}_{\epsilon}$ by more than $1 / 4$ of the decrease at $x=x_{B}$, or $\frac{\left|\eta\left(\widetilde{\widetilde{x}}_{\epsilon}, v, C A R A^{*}\right)-\eta\left(\widetilde{\widetilde{x}}_{\epsilon}, v, C A R A_{\epsilon}^{\prime}\right)\right|}{\left|\eta\left(x_{B}, v, C A R A^{*}\right)-\eta\left(x_{B}, v, C A R A_{\epsilon}^{\prime}\right)\right|}>$ 1/4. This is impossible if $\widetilde{\widetilde{x}}_{\epsilon}$ converges to $x_{A}$ because by L'Hospital's rule we have

$$
\begin{aligned}
& \lim _{\epsilon \rightarrow 0} \frac{\left|\eta\left(\widetilde{\widetilde{x}}_{\epsilon}, v, C A R A^{*}\right)-\eta\left(\widetilde{\widetilde{x}}_{\epsilon}, v, C A R A_{\epsilon}^{\prime}\right)\right|}{\left|\eta\left(x_{B}, v, C A R A^{*}\right)-\eta\left(x_{B}, v, C A R A_{\epsilon}^{\prime}\right)\right|} \\
= & \lim _{\epsilon \rightarrow 0} \frac{\frac{d}{d \epsilon}\left|\eta\left(\widetilde{\widetilde{x}}_{\epsilon}, v, C A R A^{*}\right)-\eta\left(\widetilde{\widetilde{x}}_{\epsilon}, v, C A R A_{\epsilon}^{\prime}\right)\right|}{\frac{d}{d \epsilon}\left|\eta\left(x_{B}, v, C A R A^{*}\right)-\eta\left(x_{B}, v, C A R A_{\epsilon}^{\prime}\right)\right|} \\
= & \frac{\frac{d}{d \epsilon}\left|\eta\left(\lim _{\epsilon \rightarrow 0} \widetilde{\widetilde{x}}_{\epsilon}, v, C A R A^{*}\right)-\eta\left(\lim _{\epsilon \rightarrow 0} \widetilde{\widetilde{x}}_{\epsilon}, v, C A R A_{\epsilon}^{\prime}\right)\right|}{1} \\
= & \frac{d}{d \epsilon}\left|\eta\left(x_{A}, v, C A R A^{*}\right)-\eta\left(x_{A}, v, C A R A_{\epsilon}^{\prime}\right)\right|=0,
\end{aligned}
$$

a contradiction. The same argument rules out that $\widetilde{\widetilde{x}}_{\epsilon}$ converges to $x_{C}$.

Could $\widetilde{\widetilde{x}}_{\epsilon}$ converge to $x_{B}$ ? Again, $\widetilde{\widetilde{x}}_{\epsilon}$ would need to satisfy $\left|\eta\left(\widetilde{\widetilde{x}}_{\epsilon}, v, C A R A_{\epsilon}^{\prime \prime}\right)\right|>K-\epsilon / 4$, which 
implies that as $C A R A^{*}$ is replaced by $C A R A_{\epsilon}^{\prime \prime}$ the absolute horizontal distance $|\eta(x, v, \cdot)|$ is reduced at $x=\widetilde{\widetilde{x}}_{\epsilon}$ by less than $1 / 2$ of the reduction at $x=x_{B}$. But by L'Hospital's rule we have

$$
\begin{aligned}
& \lim _{\epsilon \rightarrow 0} \frac{\left|\eta\left(\widetilde{\widetilde{x}}_{\epsilon}, v, C A R A^{*}\right)-\eta\left(\widetilde{\widetilde{x}}_{\epsilon}, v, C A R A_{\epsilon}^{\prime \prime}\right)\right|}{\left|\eta\left(x_{B}, v, C A R A^{*}\right)-\eta\left(x_{B}, v, C A R A_{\epsilon}^{\prime \prime}\right)\right|} \\
= & \lim _{\epsilon \rightarrow 0} \frac{\frac{d}{d \epsilon}\left|\eta\left(\widetilde{\widetilde{x}}_{\epsilon}, v, C A R A^{*}\right)-\eta\left(\widetilde{\widetilde{x}}_{\epsilon}, v, C A R A_{\epsilon}^{\prime \prime}\right)\right|}{\frac{d}{d \epsilon}\left|\eta\left(x_{B}, v, C A R A^{*}\right)-\eta\left(x_{B}, v, C A R A_{\epsilon}^{\prime \prime}\right)\right|} \\
= & \frac{\frac{d}{d \epsilon}\left|\eta\left(\lim _{\epsilon \rightarrow 0} \widetilde{\widetilde{x}}_{\epsilon}, v, C A R A^{*}\right)-\eta\left(\lim _{\epsilon \rightarrow 0} \widetilde{\widetilde{x}}_{\epsilon}, v, C A R A_{\epsilon}^{\prime \prime}\right)\right|}{\frac{d}{d \epsilon}\left|\eta\left(x_{B}, v, C A R A^{*}\right)-\eta\left(x_{B}, v, C A R A_{\epsilon}^{\prime \prime}\right)\right|} \\
= & \frac{\frac{d}{d \epsilon}\left|\eta\left(x_{B}, v, C A R A^{*}\right)-\eta\left(x_{B}, v, C A R A_{\epsilon}^{\prime \prime}\right)\right|}{\frac{d}{d \epsilon}\left|\eta\left(x_{B}, v, C A R A^{*}\right)-\eta\left(x_{B}, v, C A R A_{\epsilon}^{\prime \prime}\right)\right|}=1,
\end{aligned}
$$

in contradiction to the preceding sentence, so that $\widetilde{\widetilde{x}}_{\epsilon}$ cannot converge to $x_{B}$ either. Therefore, $\widetilde{X}_{\epsilon}$ must be empty under the assumptions of Case 1, for small enough $\epsilon$.

Case 2: The sets $X_{A}, X_{B}, X_{C}$ are all nonempty and $X_{B}$ is has multiple elements: In this case, define $x_{B}^{\min }=\min X_{B}$ and $x_{B}^{\max }=\max X_{B}$. The argument proceeds analogously to Case 1 , but instead of using the value $x_{B}$ in the construction, we will appropriately choose for each $\epsilon$ a value $x_{B, \epsilon} \in\left\{x_{B}^{\min }, x_{B}^{\max }\right\}$ which will determine the functions $C A R A_{\epsilon}^{\prime}$ and $C A R A_{\epsilon}^{\prime \prime}$.

For any given (small) $\epsilon>0$ consider the point $B^{\prime \min }$ with coordinates $\left(x_{B}^{\min }+K-\epsilon, v\left(x_{B}^{\min }\right)\right)$ and construct CARA functions $C A R A_{\epsilon}^{\prime \min }$ and $C A R A_{\epsilon}^{\prime \prime m i n}$ just as in Case 1 but using $x_{B}^{\min }$ instead of $x_{B}: C A R A_{\epsilon}^{\prime m i n}$ is the CARA function through $A, B^{\prime m i n}$ and $C$, and $C A R A_{\epsilon}^{\prime \prime m i n}$ is a right shift of $C A R A_{\epsilon}^{\prime \text { min }}$ by $\epsilon / 2$. Among the $x$-values in $X_{B}$, let $x_{B, \epsilon}$ be the value with the smallest reduction in horizontal distance that is achieved by replacing $C A R A^{*}$ by $C A R A_{\epsilon}^{\prime m i n}$, given by

$$
x_{B, \epsilon}=\arg \min _{x \in X_{B}}\left|\eta\left(x, v, C A R A^{*}\right)\right|-\left|\eta\left(x, v, C A R A_{\epsilon}^{\prime \min }\right)\right| .
$$

Note that $\left|\eta\left(x, v, C A R A^{*}\right)\right|-\left|\eta\left(x, v, C A R A_{\epsilon}^{\prime \min }\right)\right|$ is simply the horizontal distance between $C A R A^{*}$ and $C A R A_{\epsilon}^{\prime m i n}$ at the level $v(x)$ - it can be rewritten as $\left|\eta\left(\widetilde{x}, C A R A_{\epsilon}^{\prime \min }, C A R A^{*}\right)\right|$ for $\widetilde{x}=$ $\left(C A R A^{*}\right)^{-1}(v(x)) .{ }^{40}$ This yields that $x_{B, \epsilon} \in\left\{x_{B}^{\min }, x_{B}^{\max }\right\}$ because the two functions cross in $A$ and $C$ : for such a pair of functions, property (v) of the lemma implies that their horizontal distance cannot have a local minimum between $A$ and $C$.

\footnotetext{
${ }^{40}$ The reformulation is $\left|\eta\left(x, v, C A R A^{*}\right)\right|-\left|\eta\left(x, v, C A R A_{\epsilon}^{\prime \min }\right)\right|=\left(C A R A^{*}\right)^{-1}(v(x))-\left(C A R A_{\epsilon}^{\prime \min }\right)^{-1}(v(x))=$ $\widetilde{x}-\left(C A R A_{\epsilon}^{\prime \min }\right)^{-1}\left(C A R A^{*}(\widetilde{x})\right)=\eta\left(\widetilde{x}, C A R A^{*}, C A R A_{\epsilon}^{\prime \min }\right)$.
} 
If $x_{B, \epsilon}=x_{B}^{\min }$, then we set $C A R A_{\epsilon}^{\prime}=C A R A_{\epsilon}^{\prime \min }$ and $C A R A_{\epsilon}^{\prime \prime}=C A R A_{\epsilon}^{\prime \prime \min }$. If $x_{B, \epsilon}=x_{B}^{\max }$, then we repeat the construction of the two CARA functions, but using $x_{B}^{\max }$ instead of $x_{B}^{\min }$ : we construct $C A R A_{\epsilon}^{\prime \max }$ through $A, C$ and the point with coordinates $\left(x_{B}^{\max }+K-\epsilon, v\left(x_{B}^{\max }\right)\right)$, and we construct $C A R A_{\epsilon}^{\prime \prime m a x}$ by shifting $C A R A_{\epsilon}^{\prime \max }$ to the right by $\epsilon / 2$. Then we set $C A R A_{\epsilon}^{\prime}=C A R A_{\epsilon}^{\prime \max }$ and $C A R A_{\epsilon}^{\prime \prime}=C A R A_{\epsilon}^{\prime \prime \max }$.

Notice that if $x_{B, \epsilon}=x_{B}^{\max }, C A R A_{\epsilon}^{\prime \max }$ lies above $C A R A_{\epsilon}^{\prime \min }$ at all $x \in X_{B}: x_{B, \epsilon}=x_{B}^{\max }$ implies that the horizontal distance decreases less at $x_{B}^{\max }$ than at $x_{B}^{\min }$ when $C A R A^{*}$ is replaced by $C A R A_{\epsilon}^{\prime \min }$ - hence, the decrease at $x_{B}^{\max }$ is less than $\epsilon$. But when replacing $C A R A^{*}$ by $C A R A_{\epsilon}^{\prime \max }$, the distance at $x_{B}^{\max }$ is decreased by $\epsilon$, so that $C A R A_{\epsilon}^{\prime \max }$ lies above $C A R A_{\epsilon}^{\prime \min }$ at $x=x_{B}^{\max }$. Since two CARA functions can only cross each other twice (by property (v) of the lemma), and $C A R A_{\epsilon}^{\prime \min }$ and $C A R A_{\epsilon}^{\prime \max }$ cross in $A$ and $C, C A R A_{\epsilon}^{\prime \max }$ lies above $C A R A_{\epsilon}^{\prime \min }$ at all $x \in\left[x_{A}-\right.$ $\left.K, x_{C}-K\right]$. By an analogous argument, if $x_{B, \epsilon}=x_{B}^{\min }$ then $C A R A_{\epsilon}^{\prime \min }$ lies above $C A R A_{\epsilon}^{\prime \max }$ at all $x \in\left[x_{A}-K, x_{C}-K\right]$. Therefore, we have established that the construction of $x_{B, \epsilon}, C A R A_{\epsilon}^{\prime}$ and $C A R A_{\epsilon}^{\prime \prime}$ has the property that $\left|\eta\left(x, v, C A R A^{*}\right)\right|-\left|\eta\left(x, v, C A R A_{\epsilon}^{\prime}\right)\right| \geq \epsilon$ for all $x \in X_{B}$, and hence $\left|\eta\left(x, v, C A R A^{*}\right)\right|-\left|\eta\left(x, v, C A R A_{\epsilon}^{\prime \prime}\right)\right| \geq \epsilon / 2$ for all $x \in X_{B}$.

The arguments of Case 1 now apply to this construction, ruling out that $\left|\eta\left(x, v, C A R A_{\epsilon}^{\prime \prime}\right)\right|>$ $K-\epsilon / 4$ for small enough $\epsilon$ at all $x$ that are bounded away from $X_{B}$. In addition, we need to rule out two possibilities: First, could $\left|\eta\left(x, v, C A R A_{\epsilon}^{\prime \prime}\right)\right|>K-\epsilon / 4$ be true for $x \in X_{B}$ ? This is impossible due to the observation in the previous paragraph, which implies that $\left|\eta\left(x, v, C A R A_{\epsilon}^{\prime \prime}\right)\right| \leq K-\epsilon / 2$ for $x \in X_{B}$. Second, could there exist a sequence $\left(\epsilon, \widetilde{x}_{\epsilon}\right)$ such that $\epsilon \rightarrow 0, \widetilde{x}_{\epsilon} \in \widetilde{X}_{\epsilon}$ and $\widetilde{x}_{\epsilon}$ becomes arbitrarily close to $X_{B}$ ? No: $\widetilde{x}_{\epsilon}$ would have a subsequence $\widetilde{\widetilde{x}}_{\epsilon}$ converging to some value $\widetilde{\widetilde{x}} \in X_{B}$, and due to the observation in the previous paragraph we could use the same argument as in Case 1 , where convergence of $\widetilde{\widetilde{x}}_{\epsilon}$ to $x_{B}$ was ruled out: It would have to hold that replacing $C A R A^{*}$ by $C A R A_{\epsilon}^{\prime \prime}$ reduces $|\eta(x, v, \cdot)|$ at $x=\widetilde{\widetilde{x}}_{\epsilon}$ by less than $1 / 2$ of the reduction at $x=\widetilde{\widetilde{x}}$, which is disproven by L'Hospital's rule for small enough $\epsilon$.

Case 3: $X_{A}$ is empty but $X_{B}$ and $X_{C}$ are nonempty, so that there is only one sign change between $K$-distance values: The argument follows exactly the cases above, except that $x_{A}$ is chosen to be 


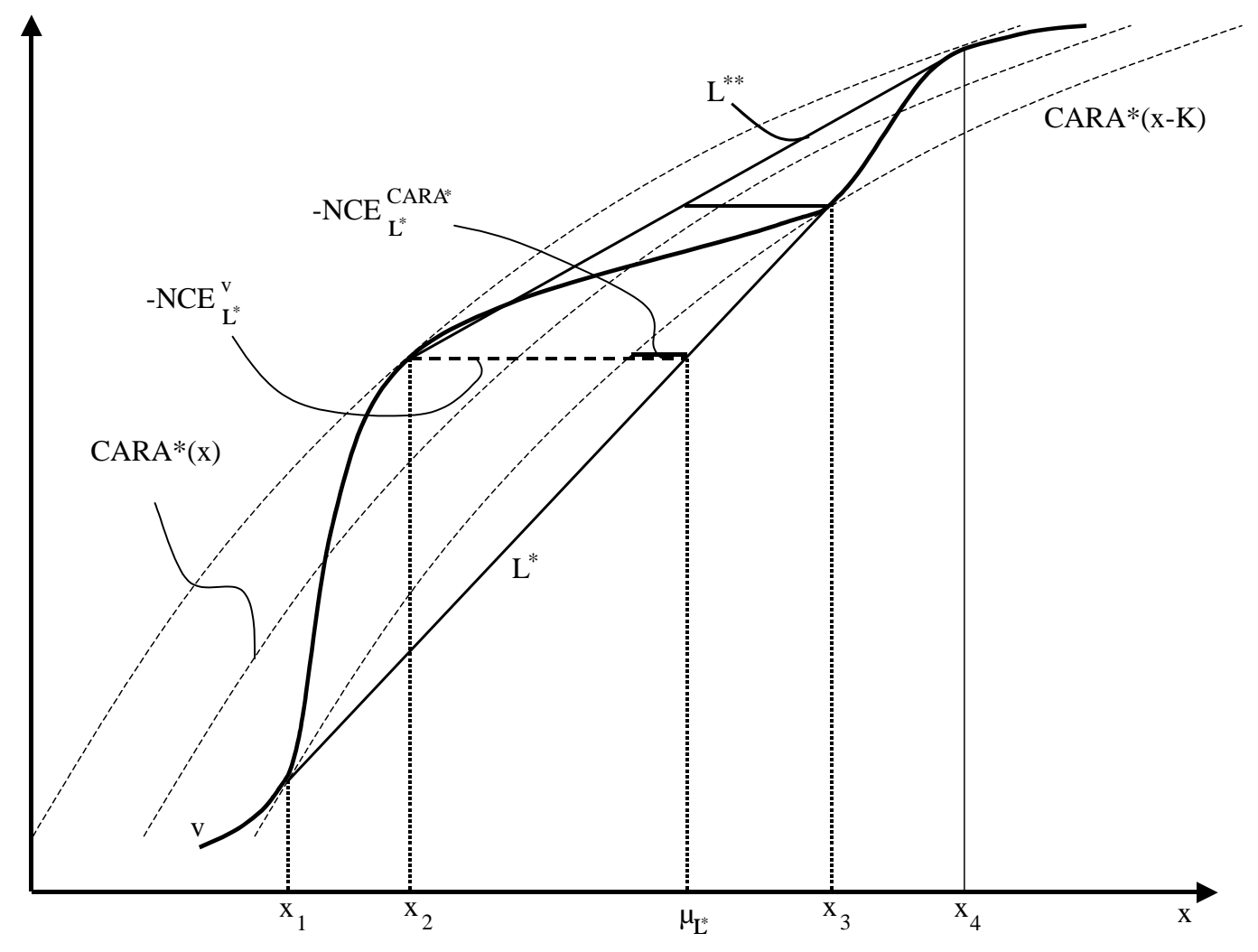

Figure 6.2: Lotteries $L^{*}$ and $L^{* *}$

the smallest possible value, $x_{A}=\underline{x}{ }^{41}$

Case 4: $X_{B}$ is empty or both $X_{A}$ and $X_{C}$ are empty, so that no sign change between $K$-distance points: in this case $C A R A^{*}$ cannot be the best-fitting CARA funcntion, as it can obviously be improved by a horizontal shift that moves it closer to $v$.

Step 2: Constructing the candidate lotteries: As seen in Proposition 1, it suffices to find a lottery that can be shifted within $[\underline{x}, \bar{x}]$ such that its $N C E$ varies by at least $K$. Step 1 guarantees the existence of four $K$-distance values $x_{1}<x_{2}<x_{3}<x_{4}$ where $v$ lies on alternating sides of $C A R A^{*}$. We start by considering the lottery $L^{*}$ in Figure 6.2 , which has as its two outcomes $x_{1}$ and $x_{3}$, and

\footnotetext{
${ }^{41}$ The case that $X_{C}$ is empty is analogous, with $x_{C}=\bar{x}$.
} 
has an expectation $\mu_{L^{*}}$ that is chosen such that the decisionmaker is indifferent between receiving lottery $L^{*}$ or a sure payment of $x_{2}$. This can be expressed as $L^{*}\left(\mu_{L^{*}}\right)=v\left(x_{2}\right)$. This lottery has the property that $N C E_{L^{*}}^{v}=N C E_{L^{*}}^{C A R A^{*}}-2 K$, as can be seen in Figure 6.2: $-N C E_{L^{*}}^{C A R A^{*}}$ is equal to the horizontal distance between $\left(\mu_{L^{*}}, L^{*}\left(\mu_{L^{*}}\right)\right)$ and the graph of $C A R A^{*}(x-K)$ at $v$-value $v\left(x_{2}\right)$ - this is because $C A R A^{*}$ 's NCE is constant across shifts of a lottery (property (ii) of the lemma), so that $N C E_{L^{*}}^{C A R A^{*}(x-K)}$ and $N C E_{L^{*}}^{C A R A^{*}(x)}$ are identical. Hence, it holds that $N C E_{L^{*}}^{v}=N C E_{L^{*}}^{C A R A^{*}}-2 K$, as the point $\left(x_{2}, v\left(x_{2}\right)\right)$ lies $2 K$ to the left of the graph of $C A R A^{*}(x-K)$.

Analogously, consider the lottery $L^{* *}$, with outcomes $x_{2}$ and $x_{4}$ and the property that $L^{* *}\left(\mu_{L^{* *}}\right)=$ $v\left(x_{3}\right)$ (see Figure 6.2). Here, it holds that $N C E_{L^{* *}}^{v}=N C E_{L^{* *}}^{C A R A^{*}}+2 K$.

Now consider changing the payoffs of lottery $L^{*}$, while holding its expected value $\mu_{L}$ constant, at the level where $L\left(\mu_{L}\right)=v\left(x_{2}\right)$. It holds that any such lottery $L$ - with payoffs $x^{\prime}$ and $x^{\prime \prime}$ that lie in $[\underline{x}, \bar{x}]$, and with $L\left(\mu_{L}\right)=v\left(x_{2}\right)$ - has the property that $N C E_{L}^{v} \leq N C E_{L}^{C A R A^{*}}$. This is because $v\left(x^{\prime}\right) \leq C A R A^{*}\left(x^{\prime}+K\right)$ and $v\left(x^{\prime \prime}\right) \leq C A R A^{*}\left(x^{\prime \prime}+K\right)$ : If $v$ reaches both of these upper bounds, then $N C E_{L}^{v}=N C E_{L}^{C A R A^{*}}$. All lower values of $v\left(x^{\prime}\right)$ and $v\left(x^{\prime \prime}\right)$ result in a lower location of the lottery line $L(\cdot)$, and hence in a smaller $N C E$.

Analogously, it holds that any binary lottery $L$ with $L\left(\mu_{L}\right)=v\left(x_{3}\right)$ has the property that $N C E_{L}^{v} \geq N C E_{L}^{C A R A^{*}}$, if both payoffs lie in $[\underline{x}, \bar{x}]$.

Notice that the above properties suggest a construction that would produce the desired result: If we can shift lottery $L^{*}$ far enough to the right so that this shift results in a lottery $\widetilde{L}^{*}$ with an expected utility of $L\left(\mu_{\widetilde{L}^{*}}\right)=v\left(x_{3}\right)$, then we are done: due to CARA's constant $N C E$ (see property (ii) of the lemma), we would have $N C E_{L^{*}}^{C A R A^{*}}=N C E_{\widetilde{L}^{*}}^{C A R A^{*}}$, so the two properties $N C E_{L^{*}}^{v}=N C E_{L^{*}}^{C A R A^{*}}-2 K$ and $N C E_{\widetilde{L}^{*}}^{v} \geq N C E_{\widetilde{L}^{*}}^{C A R A^{*}}$ would imply that the $N C E$ varies by at least $2 K$, i.e. more than we need. We could then apply Proposition 1 to conclude that an FOSD-violation by $2 K$ can be generated. The trouble is that we cannot always shift $L^{*}$ far enough. It may be that the upper payoff bound $\bar{x}$ is close or equal to $x_{4}$, and that $x_{3}$ is close to $x_{4}$, such that $x_{3}-\mu_{L^{*}}$ exceeds $\bar{x}-x_{3}$. Similarly, it may be that the analogous shift of $L^{* *}$ to the left is not possible either. 


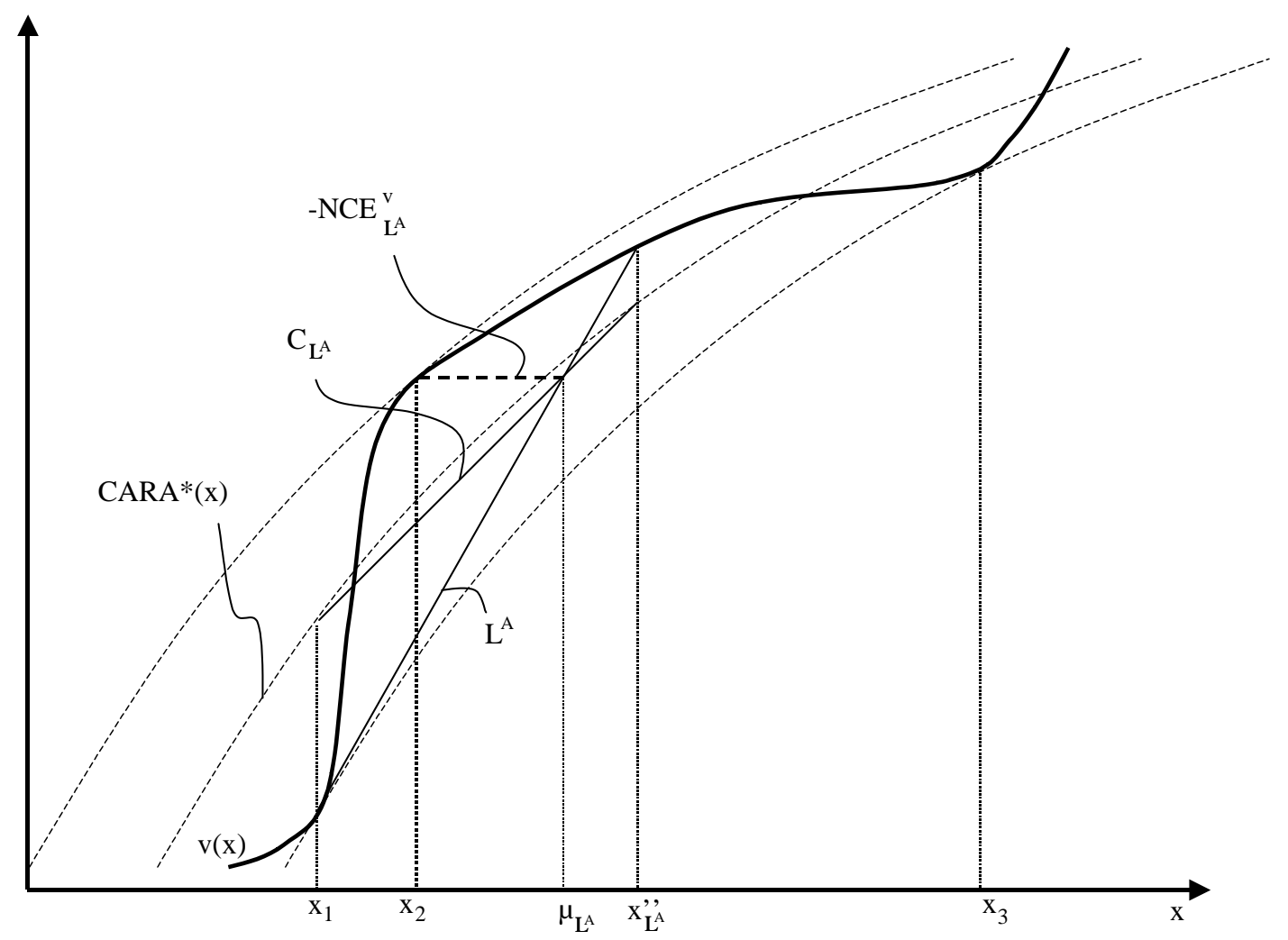

Figure 6.3: Lottery $L^{A}$, in the case $v\left(x_{1}\right)<C A R A\left(x_{1}\right)$

However, we can use the lotteries $L^{*}$ and $L^{* *}$ to generate two "shorter" lotteries that can be shifted far enough for the NCE to vary by at least $K$ (as will be shown in the next steps).

First, there exists a binary lottery $L^{A}$ with the properties that $N C E_{L^{A}}^{v}=N C E_{L^{A}}^{C A R A^{*}}-K$, that the low outcome is $x_{1}$, and that its expected utility is $L\left(\mu_{L^{A}}\right)=v\left(x_{2}\right)$. This lottery is depicted in Figure 6.3. $L^{A}$ must exist due to continuity: consider the lottery $L^{*}$, and decrease its high payoff outcome $x^{\prime \prime}$, but keep the low lottery outcome $x_{1}$ constant and keep the expected utility constant at $L(\mu)=v\left(x_{2}\right)$. At the starting point of this variation, i.e. with a high outcome of $x_{3}$, it holds that $N C E_{L^{*}}^{v}=N C E_{L^{*}}^{C A R A^{*}}-2 K$. As the high outcome approaches $x_{2}$, it holds that the $N C E$ s of $v$ and $C A R A^{*}$ of the resulting lotteries converge - they both approach 0. Hence, by continuity of $N C E^{v}$ and $N C E^{C A R A^{*}}$, there exists a value $x_{L^{A}}^{\prime \prime}$ that results in a lottery $L^{A}$ with 


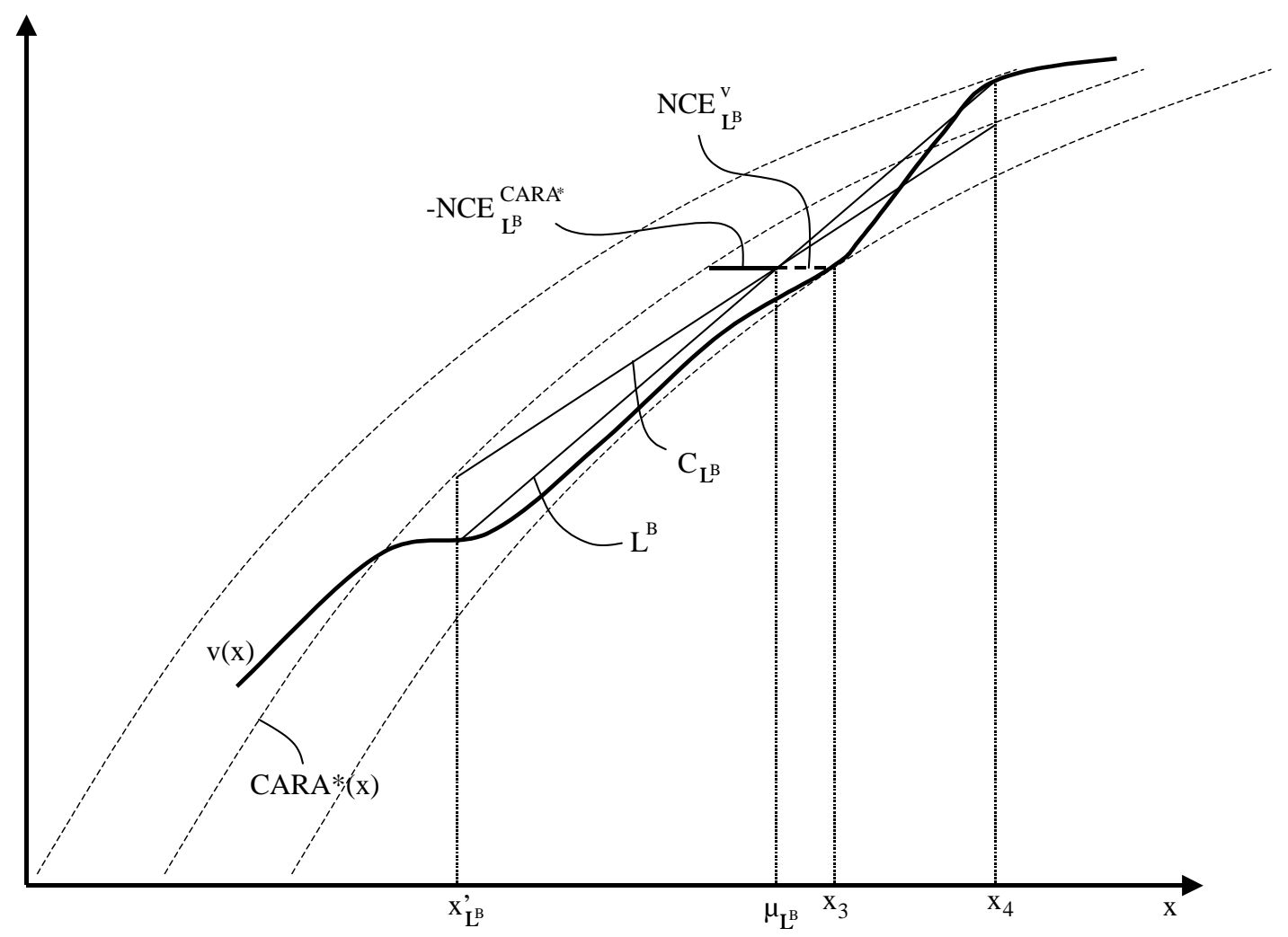

Figure 6.4: Lottery $L^{B}$, in the case $v\left(x_{1}\right)<C A R A\left(x_{1}\right)$

$N C E_{L^{A}}^{v}=N C E_{L^{A}}^{C A R A^{*}}-K$. Let $L^{A}$ be the shortest lottery that has this property.

Second, and analogously, there exists a lottery $L^{B}$ with the property $N C E_{L^{B}}^{v}=N C E_{L^{B}}^{C A R A^{*}}+$ $K$, with a high outcome of $x_{4}$, and with expected value of $L\left(\mu_{L^{B}}\right)=v\left(x_{3}\right)$. Again, let $L^{B}$ be the shortest such lottery, i.e. the one with the highest low outcome. This lottery is depicted in Figure 6.4 .

Step 3: If $C A R A^{*}$ is concave, then either $L^{A}$ or $L^{B}$ can be shifted far enough: In the following we will show that either $L^{A}$ can be shifted to the right, resulting in a lottery $L^{\widetilde{A}}$ with expected utility $L^{\widetilde{A}}\left(\mu_{L^{\widetilde{A}}}\right)=v\left(x_{3}\right)$ or $L^{B}$ can be shifted to the left, resulting in a lottery $L^{\widetilde{B}}$ with expected utility $L^{\widetilde{B}}\left(\mu_{L^{\widetilde{B}}}\right)=v\left(x_{2}\right)$. By the observations in the previous step, this suffices to prove the result 
(for the case that $C A R A^{*}$ is concave). We consider 4 cases - two with $v\left(x_{1}\right)<C A R A^{*}\left(x_{1}\right)$ (as in Figures 6.3 and 6.4), and two with $v\left(x_{1}\right)>C A R A^{*}\left(x_{1}\right)$.

Case 1: $v\left(x_{1}\right)<C A R A^{*}\left(x_{1}\right)$, and $L^{A}$ is longer than $L^{B}$, i.e. $x_{L^{A}}^{\prime \prime}-x_{1}>x_{4}-x_{L^{B}}^{\prime}$ : First notice that the line $H_{L^{A}}$ lies above the lottery line of $L^{A}$. (See Figure 6.3: $H_{L^{A}}$ is a straight line through $\left(x_{1}, v\left(x_{1}\right)\right)$ and $\left(x_{L^{A}}^{\prime \prime}, C A R A^{*}\left(x_{L^{A}}^{\prime \prime}+K\right)\right)$ - it is not drawn in the figure, to avoid having too many lines.) Hence, the value $x_{H^{A}}^{*}$, where $H_{L^{A}}$ intersects $C_{L^{A}}$, lies to the left of $\mu_{L^{A}}$, i.e. $x_{H^{A}}^{*} \leq \mu_{L^{A}}$. Similarly, for lottery $L^{B}$, consider the auxiliary straight line $H_{L^{B}}$ through $\left(x_{4}, v\left(x_{4}\right)\right)$ and $\left(x_{L^{B}}^{\prime}, C A R A^{*}\left(x_{L^{B}}^{\prime}-K\right)\right)$. $H_{L^{B}}$ lies below $C_{L^{B}}$, so it holds that $\mu_{L^{B}} \leq x_{H^{B}}^{*}$.

Now shift $L^{B}$ to the left, such that the low outcome of the resulting lottery $L^{B^{\prime}}$ is equal to $x_{1}$ - which is clearly possible without leaving $[\underline{x}, \bar{x}]$. Let $x_{L^{B^{\prime}}}^{\prime \prime}$ be the high outcome of $L^{B^{\prime}}$, and $\mu_{L^{B^{\prime}}}$ be its expected value. We will show that for this shifted lottery we have $L^{B^{\prime}}\left(\mu_{L^{B^{\prime}}}\right) \leq v\left(x_{2}\right)$, which implies the desired result, because by continuity of $v$ there must then exist another shifted version of $L^{B}, L^{\widetilde{B}}$, with $L^{\widetilde{B}}\left(\mu_{L^{\widetilde{B}}}\right)=v\left(x_{2}\right)$.

For the shifted lottery $L^{B^{\prime}}$, consider the auxiliary lines $H_{L^{B^{\prime}}}$ and $C_{L^{B^{\prime}}}$, which intersect at a point $x_{H^{B^{\prime}}}^{*}$. Due to property (iii) of the lemma, the relative $x$-location of this intersection is constant, i.e. it holds that $x_{H^{B^{\prime}}}^{*}=x_{L^{B^{\prime}}}^{\prime \prime}-\left(x_{4}-x_{H^{B}}^{*}\right)$. This implies that $\mu_{L^{B^{\prime}}} \leq x_{H^{B^{\prime}}}^{*}$, because both $x_{L^{B^{\prime}}}$ and $x_{H^{B^{\prime}}}^{*}$ were shifted by the same $\triangle x$ (from $x_{L^{B}}^{*}$ and $x_{H^{B}}^{*}$ ).

It holds that $C_{L^{A}}\left(\mu_{L^{A}}\right)=v\left(x_{2}\right)$ by construction. $x_{H^{A}}^{*} \leq \mu_{L^{A}}$ then implies that $C_{L^{A}}\left(x_{H^{A}}^{*}\right) \leq$ $v\left(x_{2}\right)$, because $C_{L^{A}}$ is increasing. From property (iv) of the lemma, it holds that $C_{L^{B^{\prime}}}\left(x_{H^{B^{\prime}}}^{*}\right)<$ $C_{L^{A}}\left(x_{H^{A}}^{*}\right): L^{A}$ is longer than $L^{B^{\prime}}$ and both have the same low outcome $x_{1}$, so the property applies here.

From above, we know that $\mu_{L^{B^{\prime}}} \leq x_{H^{B^{\prime}}}^{*}$. But for all values $x \leq x_{H^{B^{\prime}}}^{*}$ it holds that $H_{L^{B^{\prime}}}(x) \leq$ $C_{L^{B^{\prime}}}\left(x_{H^{B^{\prime}}}^{*}\right)$, because $H_{L^{B^{\prime}}}$ and $C_{L^{B^{\prime}}}$ intersect in $x_{H^{B^{\prime}}}^{*}$. Hence, $H_{L^{B^{\prime}}}\left(\mu_{L^{B^{\prime}}}\right) \leq C_{L^{B^{\prime}}}\left(x_{H^{B^{\prime}}}^{*}\right)$, and since the lottery line $L^{B^{\prime}}$ lies below $H_{L^{B^{\prime}}}$, it holds that $L^{B^{\prime}}\left(\mu_{L^{B^{\prime}}}\right) \leq H_{L^{B^{\prime}}}\left(\mu_{L^{B^{\prime}}}\right)$. Collecting the above inequalities, it holds that $L^{B^{\prime}}\left(\mu_{L^{B^{\prime}}}\right)<v\left(x_{2}\right)$.

Case 2: $v\left(x_{1}\right)<C A R A^{*}\left(x_{1}\right)$, and $L^{A}$ is shorter than $L^{B}$, i.e. $x_{L^{A}}^{\prime \prime}-x_{1} \leq x_{4}-x_{L^{B}}^{\prime}$ : Now shift lottery $L^{A}$ to the right, resulting in a lottery $L^{A^{\prime}}$ with high outcome of $x_{4}$ and expected value $\mu_{L^{A^{\prime}}}$. 


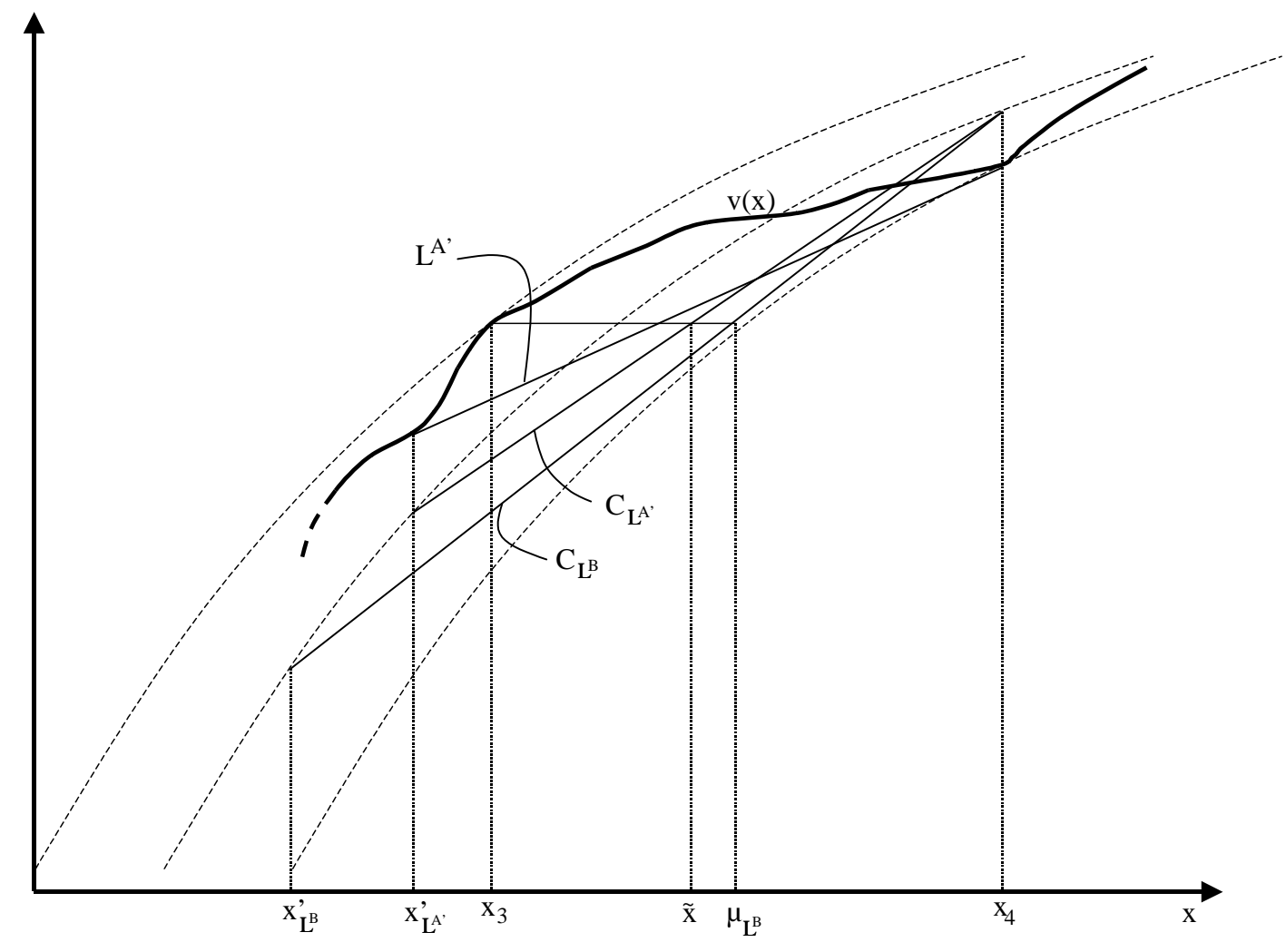

Figure 6.5: Location of $C_{L^{B}}, C_{L^{A^{\prime}}}$ and $L^{A^{\prime}}$ in Case 3 of Step 3.

Construct the auxiliary lines $H_{L^{A^{\prime}}}$ and $C_{L^{A^{\prime}}}$, which intersect at $x_{H^{A^{\prime}}}^{*}$. Analogous to the arguments in Case 1, it holds that $x_{H^{A^{\prime}}}^{*} \leq \mu_{L^{A^{\prime}}}$ and $x_{H^{B}}^{*} \geq \mu_{L^{B}}$. Also analogous to the above arguments, one can show that $C_{L^{B}}\left(x_{H^{B}}^{*}\right) \geq v\left(x_{3}\right), C_{L^{A^{\prime}}}\left(x_{H^{A^{\prime}}}^{*}\right) \geq C_{L^{B}}\left(x_{H^{B}}^{*}\right), H_{L^{A^{\prime}}}\left(\mu_{L^{A^{\prime}}}\right) \geq C_{L^{A^{\prime}}}\left(x_{H^{A^{\prime}}}^{*}\right)$, and $L^{A^{\prime}}\left(x_{L^{A^{\prime}}}^{*}\right) \geq H_{L^{A^{\prime}}}\left(\mu_{L^{A^{\prime}}}\right) \cdot{ }^{42}$ Hence, $L^{A^{\prime}}\left(\mu_{L^{A^{\prime}}}\right) \geq v\left(x_{3}\right)$, and it follows that there exists a lottery $L^{\widetilde{A}}$ with $L^{\widetilde{A}}\left(\mu_{L^{\widetilde{A}}}\right)=v\left(x_{3}\right)$.

Case 3: $C A R A^{*}\left(x_{1}\right)<v\left(x_{1}\right)$, and $L^{A}$ is shorter than $L^{B}$, i.e. $x_{L^{A}}^{\prime \prime}-x_{1} \leq x_{4}-x_{L^{B}}^{\prime}$ : Again, shift

\footnotetext{
${ }^{42}$ The following repeats the arguments, for this case. It holds that $C_{L^{B}}\left(\mu_{L^{B}}\right)=v\left(x_{3}\right)$ by construction. $x_{H^{B}}^{*} \geq \mu_{L^{B}}$ then implies that $C_{L^{B}}\left(x_{H^{B}}^{*}\right) \geq v\left(x_{3}\right)$, because $C_{L^{B}}$ is increasing. Property (iv) of the lemma yields $C_{L^{B}}\left(x_{H^{B}}^{*}\right) \leq$ $C_{L^{A^{\prime}}}\left(x_{H^{A^{\prime}}}^{*}\right): L^{B}$ is longer than $L^{A^{\prime}}$ and both have the same high outcome $x_{4}$. From above, we know that $x_{H^{A^{\prime}}}^{*} \leq \mu_{L^{A^{\prime}}}$, so that $H_{L^{A^{\prime}}}(x) \geq C_{L^{A^{\prime}}}\left(x_{H^{A^{\prime}}}^{*}\right)$. Hence, $H_{L^{A^{\prime}}}\left(\mu_{L^{A^{\prime}}}\right) \geq C_{L^{A^{\prime}}}\left(x_{H^{A^{\prime}}}^{*}\right)$, and since the lottery line $L^{A^{\prime}}$ lies above $H_{L^{A^{\prime}}}$, it holds that $L^{A^{\prime}}\left(\mu_{L^{A^{\prime}}}\right) \geq H_{L^{A^{\prime}}}\left(\mu_{L^{A^{\prime}}}\right)$.
} 
$L^{A}$ to the right, resulting in a lottery $L^{A^{\prime}}$ with high outcome $x_{4}$ and expected value $\mu_{L^{A^{\prime}}}$. Consider Figure 6.5, which depicts the auxiliary lines $C_{L^{A^{\prime}}}$ and $C_{L^{B}}$. Since both lotteries $L^{B}$ and $L^{A^{\prime}}$ have the same high outcome, $C_{L^{A^{\prime}}}$ lies above $C_{L^{B}}$. As in Case 2, we will argue that $L^{A^{\prime}}\left(\mu_{L^{A^{\prime}}}\right) \geq v\left(x_{3}\right)$. To this end, we first ask where the lottery line $L^{A^{\prime}}$ lies, relative to $C_{L^{A^{\prime}}}$. Observe that if the entire line $L^{A^{\prime}}$ lies above $v\left(x_{3}\right)$, then we are done $-L^{A^{\prime}}\left(\mu_{L^{A^{\prime}}}\right) \geq v\left(x_{3}\right)$ must be true in this case. Hence, assume to the contrary that the low payoff of $L^{A^{\prime}}$ lies below $v\left(x_{3}\right), L^{A^{\prime}}\left(x_{L^{A^{\prime}}}^{\prime}\right)<v\left(x_{3}\right)$, as in the figure. $L^{A^{\prime}}$ crosses the horizontal line at level $v\left(x_{3}\right)$ somewhere, because its high payoff is $x_{4}$, and $v$ is increasing. Furthermore, we will show in the next paragraph that at $v$-level $v\left(x_{3}\right), L^{A^{\prime}}$ necessarily lies to the left of $C_{L^{A^{\prime}}}$. Otherwise, there would exist a lottery $\widehat{L}$ that is shorter than $L^{B}$ but has all of the properties of $L^{B}$ : it would hold that $N C E_{\widehat{L}}^{v}=N C E_{\widehat{L}}^{C A R A^{*}}-K, x^{\prime \prime}(\widehat{L})=x_{4}$, and $\widehat{L}\left(\mu_{\widehat{L}}\right)=v\left(x_{3}\right)$. This would contradict the definition of $L^{B}$ as the shortest lottery with these properties.

The following shows existence of $\widehat{L}$, for this case. Consider the lottery $\widehat{\widehat{L}}$ with outcomes $x_{L^{A^{\prime}}}^{\prime}$ and $x_{L^{A^{\prime}}}^{\prime \prime}$, and an expected value $\mu_{\widehat{\widehat{L}}}$ that is chosen such that $\widehat{\widehat{L}}\left(\mu_{\widehat{\widehat{L}}}\right)=v\left(x_{3}\right)$. If $L^{A^{\prime}}$ lies to the right of $C_{L^{A^{\prime}}}$ at $v$-level $v\left(x_{3}\right)$, then it follows that $N C E_{\widehat{\widehat{L}}}^{v}<N C E_{\widehat{\widehat{L}}}^{C A R A^{*}}-K$. Hence, we can increase the lower outcome of this lottery, keeping the expected value $\mu_{\widehat{L}}$ constant, until (by continuity) there results a lottery $\widehat{L}$ with $N C E_{\widehat{L}}^{v}=N C E_{\widehat{L}}^{C A R A^{*}}-K$, as claimed in the previous paragraph.

The observation that $L^{A^{\prime}}$ lies to the left of $C_{L^{A^{\prime}}}$ at $v$-level $v\left(x_{3}\right)$ implies that there exists a value $\widetilde{x}$ where $C_{L^{A^{\prime}}}$ crosses the horizontal line at level $v\left(x_{3}\right)$, see Figure 6.5 . (This is true because otherwise $C_{L^{A^{\prime}}}$ would lie entirely above the horizontal line at $v\left(x_{3}\right)$ and could therefore not lie to the right of $L^{A^{\prime}}$ at $v\left(x_{3}\right)$.) Also, it holds that $L^{A^{\prime}}$ lies above $C_{L^{A^{\prime}}}$ at $\widetilde{x}$, i.e. $L^{A^{\prime}}(\widetilde{x}) \geq C_{L^{A^{\prime}}}(\widetilde{x})=v\left(x_{3}\right)$.

Next, construct the auxiliary lines $J_{L^{B}}$ and $J_{L^{A^{\prime}}}$, which intersect with $C_{L^{B}}$ and $C_{L^{A^{\prime}}}$, respectively, at $x_{J^{B}}^{*}$ and $x_{J^{A^{\prime}}}^{*}$. Analogous to Case 1, it holds that $x_{J^{B}}^{*} \geq \mu_{L^{B}}$ and $x_{J^{A^{\prime}}}^{*} \leq \mu_{L^{A^{\prime}}} .{ }^{43}$ The first of these inequalities implies that $C_{L^{B}}\left(x_{J^{B}}^{*}\right) \geq C_{L^{B}}\left(\mu_{L^{B}}\right)$ (because $C_{L^{B}}$ is increasing), and hence $C_{L^{B}}\left(x_{J^{B}}^{*}\right) \geq v\left(x_{3}\right)$.

\footnotetext{
${ }^{43}$ This is because $J_{L^{B}}$ lies above the lottery line $L^{B}$ and $J_{L^{A}}$ lies above $L^{A}$, and the relative locations of $x_{J^{A^{\prime}}}^{*}$ and $\mu_{L^{A^{\prime}}}$ are not affected by the shift from $L^{A}$ to $L^{A^{\prime}}$.
} 
Now, we have

$$
C_{L^{A^{\prime}}}\left(x_{J^{A^{\prime}}}^{*}\right) \geq C_{L^{B}}\left(x_{J^{B}}^{*}\right) \geq v\left(x_{3}\right)
$$

where the first inequality is implied by property (iv) of the lemma. Since $C_{L^{A^{\prime}}}$ is increasing and reaches the level $v\left(x_{3}\right)$ at $\widetilde{x}$, this implies that $x_{J^{A^{\prime}}}^{*} \geq \widetilde{x}$. Since $L^{A^{\prime}}$ is increasing and lies above $C_{L^{A^{\prime}}}$ at $\widetilde{x}$, it follows that $L^{A^{\prime}}\left(x_{J A^{\prime}}^{*}\right) \geq L^{A^{\prime}}(\widetilde{x}) \geq v\left(x_{3}\right)$. Finally, since $x_{J A^{\prime}}^{*} \leq \mu_{L^{A^{\prime}}}$ (and $L^{A^{\prime}}$ is increasing) it follows that $L^{A^{\prime}}\left(\mu_{L^{A^{\prime}}}\right) \geq v\left(x_{3}\right)$, the desired result.

Case 4: $C A R A^{*}\left(x_{1}\right)<v\left(x_{1}\right)$, and $L^{A}$ is longer than $L^{B}$, i.e. $x_{L^{A}}^{\prime \prime}-x_{1}>x_{4}-x_{L^{B}}^{\prime}$ : This is analogous to Case 3. Shift $L^{B}$ to the left, so that the resulting lottery $L^{B^{\prime}}$ has the lower outcome $x_{1}$. Then, analogous versions of all arguments for Case 3 apply, yielding $L^{B^{\prime}}\left(\mu_{L^{B^{\prime}}}\right) \leq v\left(x_{2}\right){ }^{44}$

Step 4: The previous steps show the result for the case that $C A R A^{*}$ is concave. This can be used to show that if $C A R A^{*}$ is convex, the result holds as well.

Suppose that $C A R A^{*}$ is convex. Consider the functions that describe $v$ and $C A R A^{*}$, but viewed from upside-down: $\widehat{v}=-v(-x)$ and $\widehat{C A R A}^{*}(x)=-C A R A^{*}(-x)$. Both of these mirrored functions are increasing in $x$, on the interval $[-\bar{x},-\underline{x}]$, and $\widehat{C A R A}^{*}$ is concave. Also, for any CARA function $C A R A: x \rightarrow C A R A(x)$, it holds that $\widehat{C A R A}: x \rightarrow-C A R A(-x)$ is also a CARA function, and that the horizontal distance between $v$ and $C A R A$ on $[\underline{x}, \bar{x}]$ is equal to the horizontal distance between $\widehat{v}$ and $\widehat{C A R A}$ on $[-\bar{x},-\underline{x}]$. Hence, $\widehat{C A R A} *$ is the best-fitting CARA function for $\widehat{v}$ on the interval $[-\bar{x},-\underline{x}]$, with distance $K$ (because if there were a CARA function $\widetilde{C A R A}(\cdot)$ with a lower horizontal distance, then it would also be true that $-\widetilde{C A R A}(-x)$ is a CARA function with a lower horizontal distance from $v$ on the interval $[\underline{x}, \bar{x}])$.

\footnotetext{
${ }^{44}$ The following sketches the steps: $C_{L^{B^{\prime}}}$ lies above $C_{L^{A}}$. If the entire line $L^{B^{\prime}}$ lies below $v\left(x_{2}\right)$, then $L^{B^{\prime}}\left(\mu_{L^{B^{\prime}}}\right) \leq$ $v\left(x_{2}\right)$ holds. Assume to the contrary that $L^{B^{\prime}}\left(x_{L^{B^{\prime}}}^{\prime \prime}\right)>v\left(x_{2}\right) . L^{B^{\prime}}$ crosses the horizontal line at level $v\left(x_{2}\right)$ to the right of $C_{L^{B^{\prime}}}$, because otherwise the definition of $L^{A}$ would be violated. Hence, there exists a value $\widetilde{\widetilde{x}}$ where $C_{L^{B^{\prime}}}$ crosses the horizontal line at level $v\left(x_{2}\right)$, and $L^{B^{\prime}}(\widetilde{\widetilde{x}}) \leq C_{L^{B^{\prime}}}(\widetilde{\widetilde{x}})=v\left(x_{2}\right)$. For the locations where the auxiliary lines $J_{L^{A}}$ and $J_{L^{B^{\prime}}}$ intersect with $C_{L^{A}}$ and $C_{L^{B^{\prime}}}$, respectively, it holds that $x_{J A}^{*} \leq \mu_{L^{A}}$ and $x_{J^{B^{\prime}}}^{*} \geq \mu_{L^{B^{\prime}}}$. Therefore, and from property (iv) of the lemma, we have $C_{L^{B^{\prime}}}\left(x_{J^{B^{\prime}}}^{*}\right) \leq C_{L^{A}}\left(x_{J^{A}}^{*}\right) \leq v\left(x_{2}\right)$. Since $C_{L^{B^{\prime}}}$ is increasing and reaches $v\left(x_{2}\right)$ at $\widetilde{\widetilde{x}}$, this implies that $x_{J B^{\prime}}^{*} \leq \widetilde{\widetilde{x}}$. Since $L^{B^{\prime}}$ is increasing and $x_{J^{B^{\prime}}}^{*} \geq \mu_{L^{B^{\prime}}}$, it follows that $L^{B^{\prime}}\left(\mu_{L^{B^{\prime}}}\right) \leq$ $L^{B^{\prime}}\left(x_{J B^{\prime}}^{*}\right) \leq L^{B^{\prime}}(\widetilde{\widetilde{x}}) \leq v\left(x_{2}\right)$.
} 
Hence, from the result of step 3, we know that there is a pair of shifted lotteries, with payoffs in $[-\bar{x},-\underline{x}]$, such that $\widehat{v}$ 's net certainty equivalent varies by at least $K$ between the two lotteries. This pair of lottery choice tasks can be reflected around zero, by reversing the sign of all payoffs. Since $\widehat{v}$ is the mirror image of $v$, the resulting pair of lotteries will also have the property that $v$ 's net certainty equivalent will varies by at least $K$ between the two lotteries. Also, all payoffs of the reflected lotteries lie in $[\underline{x}, \bar{x}]$. Hence, we can apply Proposition 1 to obtain the result.

\section{Lemma: Properties of CARA:}

(For notation, see the first two paragraphs of the proof of Proposition 2.)

(i) Consider a set of three points $\left\{\left(x_{1}, v_{1}\right),\left(x_{2}, v_{2}\right),\left(x_{3}, v_{3}\right)\right\}$ that is strictly monotonic: $x_{1}<$ $x_{2}<x_{3}$ and $v_{1}<v_{2}<v_{3}$. There exists a CARA function that connects the three points.

(ii) For any CARA function $C A R A(\cdot)$ and any binary lottery $L, N C E_{L}^{C A R A}$ is constant across shifted versions of $L$, where $\triangle x$ is added to both payoffs as in Section 2. I.e., $N C E_{\widetilde{L}}^{C A R A}-$ $N C E_{L}^{C A R A}=0$.

(iii) Let $(L, \widetilde{L})$ be a pair of shifted lotteries. Then $x_{H_{\tilde{L}}}^{*}-x_{\widetilde{L}}^{\prime}$ and $x_{J_{\tilde{L}}}^{*}-x_{\widetilde{L}}^{\prime}$ are both independent of the shift $\triangle x$. I.e. the relative location of $x_{H_{L}}^{*}$ and $x_{J_{L}}^{*}$ does not change if $L$ is shifted.

(iv) Let $\alpha, r>0$, so that $C A R A$ is strictly concave. Consider a pair of lotteries $(\widetilde{L}, \widetilde{\widetilde{L}})$ such that $\widetilde{\widetilde{L}}$ that is longer than $\widetilde{L}$, i.e. $x_{\widetilde{\widetilde{L}}}^{\prime \prime}-x_{\widetilde{L}}^{\prime} \geq x_{\widetilde{L}}^{\prime \prime}-x_{\widetilde{L}}^{\prime}$. If both $\widetilde{\widetilde{L}}$ and $\widetilde{L}$ have the same low payoff $\left(x_{\widetilde{\widetilde{L}}}^{\prime}=x_{\widetilde{L}}^{\prime}\right)$, then $C_{L}\left(x_{H_{\widetilde{L}}}^{*}\right) \geq C_{L}\left(x_{H_{\widetilde{L}}}^{*}\right)$ and $C_{L}\left(x_{J_{\widetilde{L}}}^{*}\right) \geq C_{L}\left(x_{J_{\widetilde{L}}}^{*}\right)$, i.e. for the longer lottery, both values $C_{L}\left(x_{H_{L}}^{*}\right)$ and $C_{L}\left(x_{J_{L}}^{*}\right)$ are at least as large. If both have the same high payoff $\left(x_{\widetilde{L}}^{\prime \prime}=x_{\widetilde{L}}^{\prime \prime}\right)$, then the reverse inequalities are true, $C_{L}\left(x_{H_{\widetilde{L}}}^{*}\right) \leq C_{L}\left(x_{H_{\widetilde{L}}}^{*}\right)$ and $C_{L}\left(x_{J_{\widetilde{L}}}^{*}\right) \leq C_{L}\left(x_{J_{\widetilde{L}}}^{*}\right)$.

(v) Consider two CARA functions $C A R A_{1}(x)=\beta_{1}-\alpha_{1} \exp \left(-r_{1} x\right)$ and $C A R A_{2}(x)=\beta_{2}-$ $\alpha_{2} \exp \left(-r_{2} x\right)$. Their horizontal distance $\eta\left(x, C A R A_{1}, C A R A_{2}\right)$ is unimodal: its derivative w.r.t. $x$ changes its sign no more than once.

Proof: (i) For a CARA function with parameters $\alpha, \beta, r$ to connect $\left(x_{1}, v_{1}\right)$ and $\left(x_{3}, v_{3}\right)$, we 
need

$$
\begin{aligned}
& v_{1}=\beta-\alpha \exp \left(-r x_{1}\right) \\
& v_{3}=\beta-\alpha \exp \left(-r x_{3}\right) .
\end{aligned}
$$

First restrict attention to the case that the CARA function is strictly concave (i.e. $\alpha, r>0$ ) and that $\beta>v_{3}$. Under these conditions, and with some algebra, the above equations imply that $\alpha$ can be expressed as a function of $\beta$,

$$
\begin{aligned}
\alpha & =\exp \left(\frac{x_{3}}{x_{3}-x_{1}} \ln \left(\beta-v_{1}\right)-\frac{x_{1}}{x_{3}-x_{1}} \ln \left(\beta-v_{3}\right)\right) \\
& =\frac{\left(\beta-v_{1}\right)^{\frac{x_{3}-x_{1}}{x_{3}}}}{\left(\beta-v_{3}\right)^{\frac{x_{1}}{x_{3}-x_{1}}}} .
\end{aligned}
$$

Plugging the first of the expressions for $\alpha$ into the equation for $v_{1}$ yields an expression for $r$, as a function of $\beta$ :

$$
r=\frac{\ln \left(\beta-v_{1}\right)-\ln \left(\beta-v_{3}\right)}{x_{3}-x_{1}}
$$

Now vary $\beta$, and ask what values can the CARA function have at $x_{2}$, if it connects $\left(x_{1}, v_{1}\right)$ and $\left(x_{3}, v_{3}\right)$. Using the above expressions for $\alpha$ and $r$, it holds that

$$
\begin{aligned}
C A R A\left(x_{2}\right) & =\beta-\alpha \exp \left(-r x_{2}\right) \\
& =\beta-\left(\beta-v_{1}\right)^{\frac{x_{3}-x_{2}}{x_{3}-x_{1}}}\left(\beta-v_{3}\right)^{\frac{x_{2}-x_{1}}{x_{3}-x_{1}}} .
\end{aligned}
$$

As $\beta$ approaches $v_{3}$ from above, this expression approaches $v_{3}$. Also, it holds that as $\beta$ approaches $\infty, r$ approaches 0 , and therefore the function becomes approximately linear between $x_{1}$ and $x_{3}$. Hence, by varying $\beta$, all points $\left(x_{2}, v_{2}\right)$ that lie above the straight line between $\left(x_{1}, v_{1}\right)$ and $\left(x_{3}, v_{3}\right)$ can be reached, by a concave CARA function that also connects $\left(x_{1}, v_{1}\right)$ and $\left(x_{3}, v_{3}\right)$.

Now, consider the points that lie below the straight line between $\left(x_{1}, v_{1}\right)$ and $\left(x_{3}, v_{3}\right)$. Notice that the upside-down images of convex CARA functions are concave CARA functions $(-C A R A(-x)$, like in step 4 of the proof of Proposition 2), so that the above argument applies: We can find a convex CARA function connecting $\left(x_{1}, v_{1}\right),\left(x_{2}, v_{2}\right)$, and $\left(x_{3}, v_{3}\right)$ iff we can find a concave CARA function connecting $\left(-x_{3},-v_{3}\right),\left(-x_{2},-v_{2}\right)$, and $\left(-x_{1},-v_{1}\right)$. This can be achieved by the above construction. 
(ii) For a binary lottery $L$ with lower payoff $x_{L}^{\prime}$, express the higher payoff as $x_{L}^{\prime \prime}=x_{L}^{\prime}+\delta^{\prime \prime}$ and the expected value as $\mu_{L}=x_{L}^{\prime}+\widehat{\delta}$. By the definition of the certainty equivalent $C E_{L}^{C A R A}$,

$$
\begin{aligned}
C A R A\left(C E_{L}^{C A R A}\right) & =\operatorname{Pr}\left(x_{L}^{\prime}\right) C A R A\left(x_{L}^{\prime}\right)+\left(1-\operatorname{Pr}\left(x_{L}^{\prime}\right)\right) C A R A\left(x_{L}^{\prime \prime}\right) \\
& =\frac{\delta^{\prime \prime}-\widehat{\delta}}{\delta^{\prime \prime}} C A R A\left(x_{L}^{\prime}\right)+\frac{\widehat{\delta}}{\delta^{\prime \prime}} C A R A\left(x_{L}^{\prime}+\delta^{\prime \prime}\right) .
\end{aligned}
$$

Using the functional form of $C A R A$, and $N C E_{L}^{C A R A}=C E_{L}^{C A R A}-\mu_{L}$, this is equivalent to

$$
\exp \left(-r\left(N C E_{L}^{C A R A}+\widehat{\delta}\right)\right)=\frac{\delta^{\prime \prime}-\widehat{\delta}}{\delta^{\prime \prime}}+\frac{\widehat{\delta}}{\delta^{\prime \prime}} \exp \left(-r \delta^{\prime \prime}\right)
$$

which implicitly defines $N C E_{L}^{C A R A}$ independent of the lottery's lower payoff $x_{L}^{\prime}$. Since shifting the lottery to $\widetilde{L}$ is equivalent to a shift in $x_{L}^{\prime}$ while keeping $\delta^{\prime \prime}$ and $\widehat{\delta}$ constant, it follows that $N C E_{L}^{C A R A}=N C E_{\widetilde{L}}^{C A R A}$.

(iii) It is straightforward to check that the relative location of $x_{H_{L}}^{*}$ is

$$
\frac{x_{H_{L}}^{*}-x_{L}^{\prime}}{x_{L}^{\prime \prime}-x_{L}^{\prime}}=\frac{e^{K r}}{e^{-r\left(x_{L}^{\prime \prime}-x_{L}^{\prime}\right)}+e^{K r}} .
$$

Since $\triangle x$ is added to both $x_{L}^{\prime \prime}$ and $x_{L}^{\prime}$, the term $\left(x_{L}^{\prime \prime}-x_{L}^{\prime}\right)$ is constant. Hence, $\frac{x_{H_{L}}^{*}-x_{L}^{\prime}}{x_{L}^{\prime \prime}-x_{L}^{\prime}}$ is constant.

Similarly, one can check that $x_{J_{L}}^{*}$ has the relative location

$$
\frac{x_{J_{L}}^{*}-x_{L}^{\prime}}{x_{L}^{\prime \prime}-x_{L}^{\prime}}=-\frac{\left(1-e^{-r K}\right)}{\left(e^{-r K}-1-e^{r K-r\left(x_{L}^{\prime \prime}-x_{L}^{\prime}\right)}+e^{-r\left(x_{L}^{\prime \prime}-x_{L}^{\prime}\right)}\right)} .
$$

This expression, too, depends only on $\left(x_{L}^{\prime \prime}-x_{L}^{\prime}\right)$, so it is constant across shifts of the lottery.

(iv) To calculate $C_{L}\left(x_{H_{L}}^{*}\right)$, first solve $\frac{x_{H_{L}}^{*}-x_{L}^{\prime}}{x_{L}^{\prime \prime}-x_{L}^{\prime}}=\frac{e^{K r}}{e^{-r\left(x_{L}^{\prime \prime}-x_{L}^{\prime}\right)}+e^{K r}}$ for $x_{H_{L}}^{*}$ :

$$
x_{H_{L}}^{*}=\frac{1}{e^{-r\left(x_{L}^{\prime \prime}-x_{L}^{\prime}\right)}+e^{K r}}\left(x_{L}^{\prime} e^{-r\left(x_{L}^{\prime \prime}-x_{L}^{\prime}\right)}+x_{L}^{\prime \prime} e^{K r}\right)
$$

The functional form of $C_{L}$ is given by

$$
C_{L}(x)=\beta-\left(\alpha \exp \left(-r x_{L}^{\prime}\right)\right)\left(1+\frac{x-x_{L}^{\prime}}{x_{L}^{\prime \prime}-x_{L}^{\prime}}\left(\exp \left(-r\left(x_{L}^{\prime \prime}-x_{L}^{\prime}\right)\right)-1\right)\right) .
$$

Plugging $x_{H_{L}}^{*}$ into this expression yields

$$
C_{L}\left(x_{H_{L}}^{*}\right)=\beta-\alpha \frac{e^{K r-r x_{L}^{\prime \prime}}+e^{-r x_{L}^{\prime \prime}}}{e^{-r\left(x_{L}^{\prime \prime}-x_{L}^{\prime}\right)}+e^{K r}} .
$$


Taking partial derivatives, one can see that the expression is increasing in both variables $x_{L}^{\prime}$ and $x_{L}^{\prime \prime}$, if $\alpha>0$ and $r>0$. Now, observe that if $\widetilde{\widetilde{L}}$ is longer than $\widetilde{L}$ and the two lotteries have the same lower payoff, then $\widetilde{\widetilde{L}}$ can be generated from $\widetilde{L}$ by increasing $x_{\widetilde{L}}^{\prime \prime}$, so the inequality $C_{L}\left(x_{H_{\widetilde{L}}}^{*}\right) \geq C_{L}\left(x_{H_{\tilde{L}}}^{*}\right)$ follows from the monotonicity of $C_{L}\left(x_{H_{L}}^{*}\right)$ in $x_{L}^{\prime \prime}$. If they have the same higher payoff, then $\widetilde{\widetilde{L}}$ can be generated from $\widetilde{L}$ by decreasing $x_{\widetilde{L}}^{\prime}$, and $C_{L}\left(x_{H_{\widetilde{L}}}^{*}\right) \leq C_{L}\left(x_{H_{\widetilde{L}}}^{*}\right)$ follows from the monotonicity in $x_{L}^{\prime}$

Similarly, one can check that the intersection of $C_{L}$ and $J_{L}$ occurs at $v$-level

$$
C_{L}\left(x_{J_{L}}^{*}\right)=\beta-\alpha e^{-r x_{L}^{\prime}}+\alpha e^{-r K} \frac{e^{-r x_{L}^{\prime}}-e^{-r x_{L}^{\prime \prime}}}{e^{-r K}+e^{-r\left(x_{L}^{\prime \prime}-x_{L}^{\prime}\right)}},
$$

and again, the partial derivatives with respect to $x_{L}^{\prime}$ and $x_{L}^{\prime \prime}$ show that the expression is increasing in both $x_{L}^{\prime}$ and $x_{L}^{\prime \prime}$, if $\alpha>0$ and $r>0$. The same reasoning as in the previous paragraph then yields the results for $C_{L}\left(x_{J_{\widetilde{L}}}^{*}\right)$ and $C_{L}\left(x_{J_{\widetilde{L}}}^{*}\right)$.

(v) The horizontal distance between $C A R A_{1}$ and $C A R A_{2}$ is given by $\eta\left(x, C A R A_{1}, C A R A_{2}\right)=$ $x-C A R A_{2}^{-1}\left(C A R A_{1}(x)\right)$. The inverse of $C A R A_{2}$ is given by $C A R A_{2}^{-1}(v)=-\frac{1}{r_{2}} \ln \left(\frac{\beta_{2}-v}{\alpha_{2}}\right)$. Hence, $\eta\left(x, C A R A_{1}, C A R A_{2}\right)=x+\frac{1}{r_{2}} \ln \left(\frac{\beta_{2}-\beta_{1}+\alpha_{1} \exp \left(-r_{1} x\right)}{\alpha_{2}}\right)$.

The statement is true because the second derivative of $\eta$ w.r.t. $x$ is

$$
\frac{d^{2}}{d x^{2}} \eta\left(x, C A R A_{1}, C A R A_{2}\right)=\frac{\left(\beta_{2}-\beta_{1}\right)\left(-r_{1}\right)^{2} \alpha_{1} \exp \left(-r_{1} x\right)}{\left(\left(\beta_{2}-\beta_{1}\right) \alpha_{1} \exp \left(-r_{1} x\right)\right)^{2}},
$$

which has a constant sign.

Proof of Proposition 3. We show a more general version of the proposition, which covers the case that choices are representable by maximizing over $m$ the expression

$$
U(m)=\int u\left(x^{I}\right) d F\left(x^{I} \mid m\right)+\sum_{i} \int v\left(x_{i}\right) d G_{i}\left(x_{i} \mid m_{i}\right) .
$$

Here, $u$ is the global evaluation of the distribution of total earnings, and $v$ is the local or narrow evaluation of wealth changes in each bracket. Both are assumed to be increasing and twice continuously differentiable. This generalizes the assumptions made in Section 2, where $v(x)=\kappa u(x)$. In the following version of the proposition, there are only two choices to be made $(I=2)$ but this too is only a simplification. As we will see, the gambles offered in brackets 1 and 2 (to be constructed 
in the proof) can be made arbitrarily small for the result to hold, so that by the continuity of $u$ and $v$ these gambles would generally not affect the choices in any other existing brackets, unless these choices were knife-edge cases. Hence, fixing $I=2$ is not very restrictive.

Proposition $3^{\prime}$ : Suppose that $v$ is not CARA (i.e. not $\beta-\alpha \exp (-r x)$, for any $(\beta, \alpha, r)$ ). Then there is a world consisting of a pair of choices $(I=2)$, where the decisionmaker violates FOSD.

Proof: We will show that there exists a pair of 50/50 lottery choices of the following kind, violating FOSD:

Reject gamble 1: A 50/50 gamble between $x_{1}-y_{1}$ and $x_{1}+z$, with $y_{1}, z>0$, versus $x_{1}$ for sure. Accept gamble 2: A 50/50 gamble between $x_{2}-y_{2}$ and $x_{2}+z$, with $y_{2}, z>0$, versus $x_{1}$ for sure. Importantly, $y_{2}>y_{1}$.

Due to $y_{2}>y_{1}$, the choice combination $\left(\right.$ reject $_{1}$, accept $\left.t_{2}\right)$ is FOS-dominated by $\left(\right.$ reject $_{2}$, accept 1$)$. We find appropriate values of $x_{1}, x_{2}, y_{1}, y_{2}, z$ in two steps.

(i) If $v$ is differentiable but not CARA, then there exist payoffs $x_{1}^{*} \neq x_{2}^{*}$ such that the ArrowPratt degree of risk aversion $r_{A P}(x)=-\frac{v^{\prime \prime}(x)}{v^{\prime}(x)}$ is different at the two locations. This can be shown by showing the reverse statement: suppose that $r_{A P}(x)$ is constant and equal to some value $r$, at all $x$. Then we have a first-order linear differential equation for the function $v^{\prime}: v^{\prime \prime}(x)+r v^{\prime}(x)=0$, which has the general solution $v^{\prime}(x)=\alpha \exp (-r x)$. Integrating yields the general solution $v(x)=$ $\beta-\alpha \exp (-r x)$, i.e. that $v$ is CARA.

(ii) Consider a fair 50/50 gamble in an arbitrary bracket $i$ : The decisionmaker can either receive a certain payoff $x_{i}$ or a 50/50 gamble between $x_{i}+\epsilon$ and $x_{i}-\epsilon$. (We will show that as $\epsilon$ gets small, the expression $\frac{v^{\prime \prime}(x)}{v^{\prime}(x)}$ will be important, and that we would need different certainty equivalents to offset the gamble at $x_{1}^{*}$ and $x_{2}^{*}$ ) Fix the choices in the other bracket at $m_{-i}$, which will generate a distribution $F_{-i}\left(\cdot \mid m_{-i}\right)$ over the sum of earnings in bracket $-i$. For any $m_{-i}$, there exists a payment $c_{i}\left(x_{i}, \epsilon, m_{-i}\right)$ that would need to be added to $x_{i}$ in order to make $x_{i}+c_{i}$ into a certainty equivalent, i.e. for the decisionmaker to be indifferent between accepting and rejecting the gamble. This payment $c_{i}\left(x_{i}, \epsilon, m_{-i}\right)$ is implicitly defined by $U\left(x+c_{i}\left(x_{i}, \epsilon, m_{-i}\right) \mid\right.$ reject $\left._{i}, m_{-i}\right)=U\left(x \mid\right.$ accept $\left.t_{i}, m_{-i}\right)$. 
Using the global-plus-local form, this is

$$
\begin{aligned}
& \int u\left(x+x_{i}+c_{i}\left(x_{i}, \epsilon, m_{-i}\right)\right) d F_{-i}\left(x \mid m_{-i}\right)+v\left(x_{i}+c_{i}\left(x_{i}, \epsilon, m_{-i}\right)\right) \\
& -\int \frac{1}{2} u\left(x+x_{i}+\epsilon\right)+\frac{1}{2} u\left(x+x_{i}-\epsilon\right) d F_{-i}\left(x \mid m_{-i}\right)-\frac{1}{2}\left[v\left(x_{i}+\epsilon\right)+v\left(x_{i}-\epsilon\right)\right] \\
= & 0 .
\end{aligned}
$$

With fix $x_{i}$ and $m_{-i}$, we can view this as $h\left(\epsilon, c_{i}(\epsilon)\right)=0$, and apply the implicit function theorem to find that

$$
\begin{aligned}
\frac{d c_{i}}{d \epsilon} & =-\frac{(\partial h / \partial \epsilon)}{\left(\partial h / \partial c_{i}\right)} \\
& =\frac{\int \frac{1}{2} u^{\prime}\left(x+x_{i}+\epsilon\right)-\frac{1}{2} u^{\prime}\left(x+x_{i}-\epsilon\right) d F_{-i}\left(x \mid m_{-i}\right)+\frac{1}{2}\left[v^{\prime}\left(x_{i}+\epsilon\right)-v^{\prime}\left(x_{i}-\epsilon\right)\right]}{\int u^{\prime}\left(x+x_{i}+c_{i}(\epsilon)\right) d F_{-i}\left(x \mid m_{-i}\right)+v^{\prime}\left(x_{i}+c_{i}(\epsilon)\right)} .
\end{aligned}
$$

Now consider the pair of brackets labelled 1 and 2, and simultaneously offer such a 50/50 gamble to win or lose $\epsilon$ in both of them, starting from sure payoffs of $x_{1}^{*}$ and $x_{2}^{*}$ (taken from part (i) above). Now, since the decisionmaker can either accept or reject the gamble in the other bracket $j$ (with $j \in\{1,2\}$ and $j \neq i$ ), we have to consider two cases for $m_{-i}$ in the expression for $\frac{d c_{i}}{d \epsilon}$. But differentiating both of these expressions once again with respect to $\epsilon$, and taking the limit $\epsilon \rightarrow 0$, it is straighforward to see that for both possible choices $m_{j} \in\left\{\right.$ accept $_{j}$, reject $\left._{j}\right\}$, the second derivative of $c_{i}(\epsilon)$ has the same limit:

$$
\lim _{\epsilon \rightarrow 0} \frac{d}{d \epsilon} \frac{d c_{i}}{d \epsilon}\left(\text { accept }_{j}\right)=\lim _{\epsilon \rightarrow 0} \frac{d}{d \epsilon} \frac{d c_{i}}{d \epsilon}\left(\text { reject }_{j}\right)=\frac{u^{\prime \prime}\left(x_{i}^{*}+x_{j}^{*}\right)+v^{\prime \prime}\left(x_{i}^{*}\right)}{u^{\prime}\left(x_{i}^{*}+x_{j}^{*}\right)+v^{\prime}\left(x_{i}^{*}\right)}
$$

(The complete expressions for $\frac{d}{d \epsilon}\left(\frac{d c}{d \epsilon}\right)$, evaluated separately at $m_{j} \in\left\{\right.$ accept $_{j}$, reject $\left._{j}\right\}$, are available upon request.)

Now compare this expression between $i=1$ and $i=2$. In the denominator and in the numerator, respectively, the same expressions $u^{\prime \prime}\left(x_{i}^{*}+x_{j}^{*}\right)$ and $u^{\prime}\left(x_{i}^{*}+x_{j}^{*}\right)$ are added in both brackets. But $\frac{v^{\prime \prime}\left(x_{1}^{*}\right)}{v^{\prime}\left(x_{1}^{*}\right)} \neq \frac{v^{\prime \prime}\left(x_{2}^{*}\right)}{v^{\prime}\left(x_{2}^{*}\right)}$ by the result of (i). Hence, there is an $\bar{\epsilon}$ such that the second derivative of $c_{i}\left(x_{i}, \epsilon, m_{-i}\right)$ is different between $i=1$ and $i=2$, for all $\epsilon<\bar{\epsilon}$. This holds irrespective of the behavior in bracket $j \neq i$, because the limit of $\frac{d}{d \epsilon} \frac{d c_{i}}{d \epsilon}$ is the same for both possible values of $m_{j}$. I.e., even if the choice in bracket $j$ changes arbitrarily often as we send $\epsilon$ to 0 , it is still true that 
$\frac{d}{d \epsilon} \frac{d c_{i}}{d \epsilon}$ approaches a different limit in the two brackets. Hence, there is an $\overline{\bar{\epsilon}}<\bar{\epsilon}$ such that the certainty equivalent itself is different, i.e. $c_{1}\left(x_{1}^{*}, \overline{\bar{\epsilon}}\right) \neq c_{2}\left(x_{2}^{*}, \overline{\bar{\epsilon}}\right)$, because if two functions have different continuous second derivatives over an interval, then they have a different value somewhere in that interval. Wlog, assume $c\left(x_{1}^{*}, \overline{\bar{\epsilon}}\right)<c\left(x_{2}^{*}, \overline{\bar{\epsilon}}\right)$. Then, by the definition of a certainty equivalent and by monotonicity of $u$ and $v$, there exists a value $c$ with $c\left(x_{1}^{*}, \overline{\bar{\epsilon}}\right)<c<c\left(x_{2}^{*}, \overline{\bar{\epsilon}}\right)$, such that the decisionmaker strictly prefers to get $x_{1}^{*}+c$ for sure over the 50/50 gamble between $x_{1}^{*}+\overline{\bar{\epsilon}}$ and $x_{1}^{*}-\overline{\bar{\epsilon}}$, and he strictly prefers the 50/50 gamble between $x_{2}^{*}+\overline{\bar{\epsilon}}$ and $x_{2}^{*}-\overline{\bar{\epsilon}}$ over $x_{2}^{*}+c$ for sure. Hence, the desired payoffs exist: pick a $\delta$ that is small enough to not change these choices, and set $x_{1}=x_{1}^{*}+c, x_{2}=x_{2}^{*}+c, y_{1}=\overline{\bar{\epsilon}}+c, y_{2}=\overline{\bar{\epsilon}}+c+\delta, z=\overline{\bar{\epsilon}}-c$.

\section{Appendix 2: Part II of the laboratory experiment}

The first two treatments of the laboratory experiment, (Incentives-Small Scale and Flat FeeSmall Scale) were conducted with analogous second parts, which differed only with respect to real/hypothetical payments. In each of the two treatments, the first set of sessions (sessions 1 and 2 in the first treatment, and sessions 5 and 6 in the second treatment) first involved the Example 1 choices described in the text, and then a single investment choice for each participant, which followed the design of Redelmeier and Tversky (1992). Each participant was randomly designed to one of the following three choices (presented to the participants without the labels in brackets, and with the sentence in curly parentheses only for participants in the first treatment):

[One Gamble:] You now have the opportunity to play a gamble that offers a 50\% chance to win $£ 4$ and a $50 \%$ chance to lose $£ 2$. \{The gamble will be payoff relevant, i.e. all gains and losses will be added to your overall payment.\} Which of the following do you choose?

[Y1.] Play the gamble.

[N1.] Do not play the gamble.

[Five v Six Gambles:] You now have the opportunity to play a gamble that offers a $50 \%$ chance to win $£ 4$ and a $50 \%$ chance to lose $£ 2$. 
You can play the gamble several times, not just once. For each gamble that you play ( $50 \%$ chance to win $£ 4$ and $50 \%$ to lose $£ 2$ ), there will be a separate coin flip. Which of the following do you choose?

[Y2.] Play the gamble six times.

[N2.] Play the gamble five times.

[Five v Five-Plus-One Gambles:] You now have the opportunity to play a gamble that offers a 50\% chance to win $£ 4$ and a $50 \%$ chance to lose $£ 2$.

You can play the gamble several times, not just once. For each gamble that you play, there will be a separate coin flip.

You will play the gamble ( $50 \%$ chance to win $£ 4$ and $50 \%$ chance to lose $£ 2)$ five times, but you do not yet know your wins and losses. Which of the following do you choose?

[Y3.] Play the gamble for the sixth time.

[N3.] Do not play the gamble for the sixth time.

In the remaining Incentives-Small Scale sessions 3 and 4, after the Example 1 choices, the participants received a sheet that (i) announced an increase in the show-up fee by £10, and (ii) reminded them of the overall expected payoff of $£ 22$. Then they faced the following two choices, similar to Example 2:

[Example 2':]You face the following pair of concurrent decisions. First examine both decisions, then indicate your choices, by circling the corresponding letter. Both choices will be payoff relevant, i.e. the gains and losses will be added to your overall payment.

Decision 3: Choose between

E. a sure gain of $£ 0.50$

F. a $50 \%$ chance to gain $£ 6.00$ and a $50 \%$ chance to lose $£ 4.00$.

Decision 4: Choose between

G. a sure loss of $£ 5.00$

H. a $50 \%$ chance to lose $£ 10.00$, and a $50 \%$ chance to lose $£ 0.00$.

In the remaining session of the Flat Fee-Small Scale treatment, the participants also recieved an additional sheet after the Example 1 choices, which reminded them of (i) the fact that all payments were hypothetical, and (ii) that the participation fee was $£ 22$. Then they faced the same two choices 
of Example 2', except for the sentence concerning the payoff relevance. As the final two sheets in both treatments, the participants received the math problems and the personal questionnaire.

Table 7.1 summarizes the choice frequencies of these two treatments, split up according to the different choices in the second part.

\begin{tabular}{|c|c|c|c|c|c|c|c|c|c|c|c|c|}
\hline Sessions & \# of obs. & $\mathrm{A}$ and $\mathrm{C}$ & $A$ and $D$ & $\mathrm{~B}$ and $\mathrm{C}$ & $B$ and $D$ & Y 1 & Y 2 & Y 3 & $\mathrm{E}$ and $\mathrm{G}$ & $\mathrm{E}$ and $\mathrm{H}$ & $\mathrm{F}$ and $\mathrm{G}$ & $\mathrm{F}$ and $\mathrm{H}$ \\
\hline 1,2 & 33 & 0.18 & 0.24 & 0.15 & 0.42 & $11 / 11$ & $8 / 11$ & $8 / 11$ & - & - & - & - \\
\hline 3,4 & 20 & 0.25 & 0.35 & 0.05 & 0.35 & - & - & - & 0.05 & 0.00 & 0.35 & 0.60 \\
\hline 5,6 & 35 & 0.17 & 0.37 & 0.11 & 0.34 & $11 / 12$ & $12 / 12$ & $11 / 11$ & - & - & - & - \\
\hline 7 & 9 & 0.13 & 0.52 & 0.30 & 0.36 & - & - & - & 0.00 & 0.11 & 0.11 & 0.78 \\
\hline
\end{tabular}

In the the first two sessions of the third treatment (sessions 8,9), Incentives-Small Scale-Broad Presentation, the participants first made the four-way choice of Example 1, then they received a sheet that announced an increase in the show-up fee by £10, then they filled in the personal questionnaire, then they received the following choices, which are analogous to Example 2 (but with smaller payoffs): ${ }^{45}$

[Example 2":]You face the following pair of concurrent decisions. First examine both decisions, then indicate your choices, by circling the corresponding letter. Both choices will be payoff relevant, i.e. the gains and losses will be added to your overall payment.

Decision 2: Choose between

E. a sure gain/loss of $£ 0.00$

F. a $50 \%$ chance to gain $£ 6.00$ and a $50 \%$ chance to lose $£ 5.00$

Decision 3: Choose between

G. a sure loss of $£ 5.00$

H. a $50 \%$ chance to gain/lose $£ 0.00$, and a $50 \%$ chance to lose $£ 10.00$

Finally, the participants received the math problems.

In the remaining two sessions of this treatment (sessions 10,11), the procedure was the same, but

\footnotetext{
${ }^{45}$ The order of choice options within the two decisions was randomized across participants in this treatment, also in this part of the experiment (cf. footnote 14). In the other treatments, the order was always constant.
} 
instead of the Example 2" choices, the participants received three sheets asking for their certainty equivalents for the three $50 / 50$ gambles between $-£ 10.00$ and $£ 0.00,-£ 5.00$ and $£ 5.00$, and $£ 0.00$ and $£ 10.00$. For the example of the second of these three tasks, the wording was the following:

[CE for $\{-5,5\}:]$ Consider the following uncertain gain/loss:

U: $\quad$ a $50 \%$ chance to gain $£ 5.00$ and a $50 \%$ chance to lose $£ 5.00$

Also consider the following fixed gain/loss:

V: $\quad$ a sure gain/loss of $£ Z$

The exact value of $\mathrm{Z}$ is randomly chosen at the end of the experiment: All multiples of $£ 0.10$ that lie between $-£ 4.90$ and $+£ 5.00$ are equally likely. Negative numbers indicate that you lose, and positive numbers indicate that you gain.

Make the following choice:

I want to make the fixed gain/loss $\mathrm{V}$ if the gain/loss $£ Z$ is higher than $£_{-}{ }_{-}-$$_{-}$(insert a number between -5.00 and +5.00 ). If $£ Z$ is lower than or equal to that, I want to make the uncertain loss $\mathrm{U}$.

In the Flat Fee-Large Scale treatment (sessions 12, 13, 14 and 15), the procedure was analogous to sessions 8,9 of the Incentives-Small Scale-Broad Presentation treatment, except for differences with respect to paid/hypothetical payments and small/large payoff scale: The participants first made their (separately presented) Example 1 choices, then received a sheet that reminded them of the show-up fee, then filled in the personal questionnaire, then made the same choices as in Example 2", but with all payoff numbers mulitplied by 100, and finally answered the math problems.

Table 7.2 summarizes the lottery choice frequencies in the last two treatments. Table 7.3 reports the mean responses in the CE tasks of sessions 10 and 11 (with standard deviation in parentheses), as well as the mean across participants of the variation in the participant's net certainty equivalents between two lotteries (reported for two of the three pairs of lotteries). In each participant's case, 
a value of 0 would indicate constant absolute risk aversion between the two lotteries.

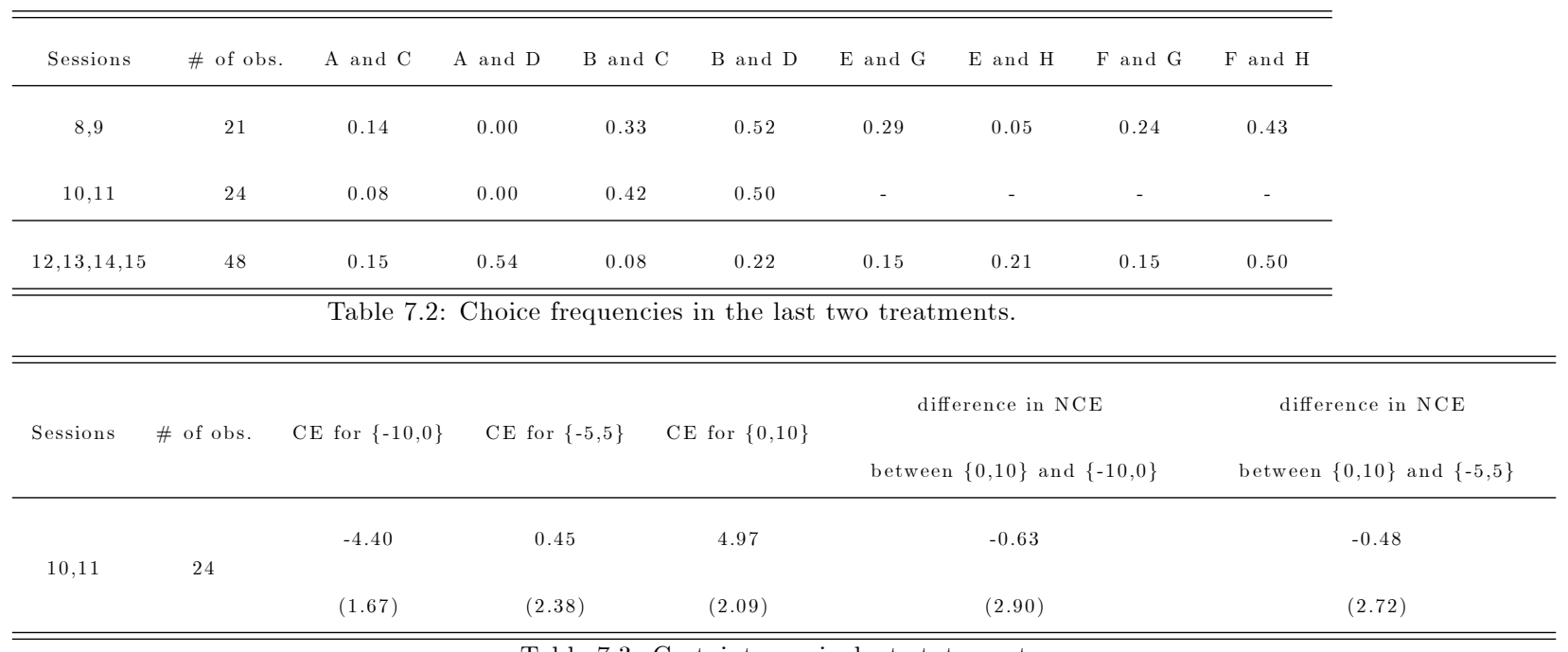

Table 7.3: Certainty equivalent statements.

\section{Appendix 3: Certainty-equivalent elicitation in the survey ex- periment}

The following are the instructions for eliciting the certainty equivalents for the $50 / 50$ lottery between $\$ 850$ and $\$ 2350$.

Decision 1: Consider the following:

Q. You have a $50 \%$ chance of winning $\$ 850$ and a $50 \%$ chance of winning $\$ 2350$

R. You will be given some unknown amount of money

What is the smallest amount of money that you would need to be given in option $\mathrm{R}$ for you to select that option over taking your chances with option Q?

Rules:

Before the study began, an unknown amount between $\$ 850$ and $\$ 2350$ was determined for option F. If this unknown amount is higher than your answer, then you will receive the unknown amount. If the unknown amount is lower than or equal to your answer, you will receive option E.

All amounts between $\$ 850$ and $\$ 2350$ are possible and equally likely, as the unknown amount. 
The results of this and the elicitation tasks for the other lotteries are summarized in the following two tables, separated into two groups: First, 7 tasks whether multiples of $\$ 100$ were used as outcomes and their spread was $\$ 1000$, and second, 4 tasks where multiplies of $\$ 50$ were used and the payoff spread was $\$ 1500$.

\begin{tabular}{|c|c|c|c|c|c|}
\hline Lot. payoffs & Treatment & $\% \min$ & $\begin{array}{l}\text { Mean of } \\
\text { stated NCE }\end{array}$ & $\begin{array}{l}\% \text { risk } \\
\text { neutral }\end{array}$ & $\% \max$ \\
\hline$-1500,-500$ & & 0.05 & 354.53 & 0.08 & 0.62 \\
\hline$-1000,0$ & & 0.03 & 303.51 & 0.15 & 0.16 \\
\hline$-1000,0$ & & 0.05 & 265.40 & 0.15 & 0.13 \\
\hline$-500,500$ & & 0.07 & 139.90 & 0.03 & 0.35 \\
\hline$-500,500$ & & 0.04 & 132.93 & 0.05 & 0.22 \\
\hline 0,1000 & & 0.01 & -66.70 & 0.38 & 0.12 \\
\hline 0,1000 & & 0.03 & -141.91 & 0.26 & 0.10 \\
\hline 0,1000 & & 0.05 & -175.95 & 0.30 & 0.04 \\
\hline 500,1500 & & 0.38 & -236.74 & 0.21 & 0.06 \\
\hline 1000,2000 & & 0.44 & -229.66 & 0.26 & 0.08 \\
\hline 1500,2500 & & 0.41 & -195.82 & 0.26 & 0.11 \\
\hline Lot. payoffs & Treatment & $\% \min$ & $\begin{array}{l}\text { Mean of } \\
\text { stated NCE }\end{array}$ & $\begin{array}{l}\% \text { risk } \\
\text { neutral }\end{array}$ & $\% \max$ \\
\hline$-1400,100$ & & 0.02 & 567.43 & 0.01 & 0.42 \\
\hline$-650,850$ & & 0.04 & 84.41 & 0.05 & 0.15 \\
\hline 100,1600 & & 0.24 & -259.52 & 0.01 & 0.09 \\
\hline 850,2350 & & 0.28 & -377.73 & 0.04 & 0.07 \\
\hline 850,2350 & & 0.27 & -381.2 & 0.03 & 0.06 \\
\hline
\end{tabular}

Table 8.2: Stated certainty equivalents of 50/50 lotteries with payoff spread $\$ 1500$. 
The means of the respondents' stated net certainty equivalents for the 11 50/50 lotteries are reported in the fourth columns of Tables 8.1 and 8.2, alongside with some other statistics of the elicited distributions. The tables show that the reported certainty equivalents vary widely as the lotteries are shifted across the interval $[-\$ 1500, \$ 2500]$, suggesting (together with Proposition 1) a large scope for FOSD violations that are due to the variablility of risk attitudes. The results systematically indicate a preference for risk taking in the domain of losses, and a strong degree of risk aversion in the gains domain.

However, we note that these data are probably unreliable. It seems doubtful that all or even most of the participants have sufficiently well understood the incentive-compatible random-price procedure. Severe limits in understanding are indicated by the large frequencies of extreme responses, as indicated in the columns labelled "\% min" and "\% max" of the tables: On average over the tasks, $32 \%$ of the responses lie at the boundaries of the possible response sets, which indicates that a large proportion may not have given an honest response, because one would need highly unusual preferences to indicate that one is indifferent between a monetary payoff $x$ for sure and a $50 / 50$ lottery between $x$ and $x+y$, where $y \neq 0$. Though this high frequency does not imply that there is no useful information in the responses of the certainty equivalence tasks, it is difficult to draw conclusions from data that are potentially severely biased.

\section{Appendix 4: Regression analysis of choices on background vari- ables}

We use the variables descried in Section 4.1.2 as our main explanatory variables of interest. At least the first three of these variables (gender, age, racial/ethnic background) can safely be viewed as exogenous so that in addition to regressions on the full set of variables, we add a set of regressions where the dependent variables are regressed on each variable alone, with no other person-specific control variables. ${ }^{46}$ In all regressions, we also include a dummy variable for the experimental

\footnotetext{
${ }^{46}$ The following are the variables that we used in the regressions with all regressors: gender, performance in the three maths questions (4 categories, for 0-3 correct answers), dummy for self-reported attendance of a maths course
} 
treatment, because the tasks were different between the treatments.

As dependent variable, we first take a dummy that indicates whether the decisionmaker chose a combination of lotteries that is FOS-dominated by another available combination (an $A$ and $D$ choice). Further, we run three additional regressions with alternative left-hand side variables: Dummies that indicate whether (i) the decisionmaker made a weakly risk averse choice in the gains domain (i.e. she chose a sure positive outcome over a lottery that has two positive outcomes and weakly higher expected value), (ii) whether she made a weakly risk averse choice in the losses domain, ${ }^{47}$ and (iii) whether she made a loss-averse choice, i.e. she was risk averse choice with regard to a lottery that had one positive and one negative outcome. Comparisons between these regressions will indicate whether any between-group differences in FOSD violations can be explained by differences in the revealed degrees of risk aversion between the groups. In each regression, we include only those decisionmakers who had the respective option available to generate both possible values of the dependent dummy variable, i.e. who faced at least one relevant decision. Tables 9.1-9.6 show the resulting marginal odds ratios from logistic regressions.

at college, age, education level (4 categories), racial/ethnic background (5 categories), region of residence in the U.S. (4 categories), household size, marital status ( 5 categories), dummy for living in a metropolitan area, log income (20 brackets), housing status (3 categories, rent/own/do not pay for housing), employment status (9 categories).

${ }^{47}$ Observations of Decision 2 in Example 4 are counted for this variable, althought the high payoff of the risky option lies above 0 . We decided to include this decision as the bevavior is arguably driven mostly by the risk attitudes below 0. These decisions are not counted for the next category, i.e. decisions that indicate degrees of loss aversion. 


\begin{tabular}{|c|c|c|c|c|c|c|c|c|}
\hline \multirow[t]{2}{*}{ Dependent var } & \multicolumn{2}{|c|}{ Dominated } & \multicolumn{2}{|c|}{ R. av. - gains } & \multicolumn{2}{|c|}{ R. av. - losses } & \multicolumn{2}{|c|}{ Loss av. } \\
\hline & (1) & $(2)$ & (3) & (4) & $(5)$ & $(6)$ & (7) & $(8)$ \\
\hline \multirow[t]{2}{*}{ female (odds ratio) } & 1.01 & 1.00 & 1.11 & 1.11 & 1.13 & 1.21 & 1.05 & 1.20 \\
\hline & $(0.14)$ & $(0.14)$ & $(0.20)$ & $(0.22)$ & $(0.16)$ & 0.19 & $(0.22)$ & $(0.29)$ \\
\hline controls & no & yes & no & yes & no & yes & no & yes \\
\hline treatment dummies & yes & yes & yes & yes & yes & yes & yes & yes \\
\hline Mean of dep. var & 0.501 & 0.500 & 0.742 & 0.741 & 0.350 & 0.356 & 0.728 & 0.728 \\
\hline Pseudo-R^2 & 0.026 & 0.062 & 0.005 & 0.048 & 0.037 & 0.066 & 0.004 & 0.089 \\
\hline \# of obs. & 916 & 914 & 643 & 641 & 926 & 924 & 471 & 471 \\
\hline
\end{tabular}

Table 9.1: Logistic regressions on gender dummy.

Note: Robust standard error in parentheses. *: $p=0.1,{ }^{* *}: p=0.05,{ }^{* * *}: p=0.01$.

\begin{tabular}{|c|c|c|c|c|c|c|c|c|c|}
\hline \multirow[t]{2}{*}{ Dependent var } & \multicolumn{3}{|c|}{ Dominated } & \multicolumn{2}{|c|}{ R. av. - gains } & \multicolumn{2}{|c|}{ R. av. - losses } & \multicolumn{2}{|c|}{ Loss av. } \\
\hline & (1) & $(2)$ & (3) & (4) & $(5)$ & (6) & (7) & $(8)$ & $(9)$ \\
\hline age (odds ratio) & $1.010^{* *}$ & $1.038^{*}$ & 1.008 & 1.004 & 1.006 & $0.992^{*}$ & 1.001 & 0.995 & 0.999 \\
\hline \multirow{3}{*}{$\operatorname{age}^{\wedge} 2$} & $(0.004)$ & $(0.024)$ & $(0.007)$ & $(0.006)$ & $(0.010)$ & $(0.004)$ & $(0.007)$ & $(0.006)$ & $(0.011)$ \\
\hline & - & 0.9997 & - & - & - & - & - & - & - \\
\hline & & $(0.0002)$ & - & - & - & - & - & - & - \\
\hline controls & no & no & yes & no & yes & no & yes & no & yes \\
\hline module dummies & yes & yes & yes & yes & yes & yes & yes & yes & yes \\
\hline Mean of dep. var & 0.501 & 0.501 & 0.500 & 0.742 & 0.741 & 0.350 & 0.356 & 0.728 & 0.728 \\
\hline Pseudo- $R^{\wedge} 2$ & 0.030 & 0.032 & 0.062 & 0.006 & 0.048 & 0.039 & 0.066 & 0.005 & 0.089 \\
\hline \# of obs. & 916 & 916 & 914 & 643 & 641 & 926 & 924 & 471 & 471 \\
\hline
\end{tabular}

Table 9.2: Logistic regressions on age.

Note: Robust standard error in parentheses. ${ }^{*}: p=0.1,{ }^{* *}: p=0.05,{ }^{* * *}: p=0.01$. 


\begin{tabular}{|c|c|c|c|c|c|c|c|c|}
\hline \multirow[t]{2}{*}{ Dependent var } & \multicolumn{2}{|c|}{ Dominated } & \multicolumn{2}{|c|}{ R. av. - gains } & \multicolumn{2}{|c|}{ R. av. - losses } & \multicolumn{2}{|c|}{ Loss av. } \\
\hline & (1) & $(2)$ & (3) & (4) & $(5)$ & (6) & (7) & (8) \\
\hline \multirow[t]{2}{*}{ black (odds ratio) } & $0.67^{*}$ & 0.69 & 0.92 & 1.21 & 1.18 & 1.38 & $0.50 * *$ & 0.60 \\
\hline & $(0.15)$ & $(0.17)$ & $(0.29)$ & $(0.43)$ & $(0.28)$ & $(0.36)$ & $(0.16)$ & $(0.23)$ \\
\hline \multirow[t]{2}{*}{ hispanic } & $0.49 * * *$ & $0.60 * *$ & $0.49 * * *$ & $0.62 *$ & 0.97 & 0.98 & $0.38 * * *$ & 0.60 \\
\hline & $(0.11)$ & $(0.15)$ & $(0.13)$ & $(0.18)$ & $(0.23)$ & $(0.25)$ & $(0.12)$ & $(0.22)$ \\
\hline \multirow[t]{2}{*}{$2+$ races, non-hispanic } & 0.94 & 0.93 & 2.13 & 2.01 & 1.14 & 1.25 & 1.06 & 1.52 \\
\hline & $(0.32)$ & $(0.33)$ & $(1.36)$ & $(1.30)$ & $(0.42)$ & $(0.46)$ & $(0.69)$ & $(0.92)$ \\
\hline \multirow[t]{2}{*}{ other, non-hispanic } & 0.69 & 0.80 & 0.48 & 0.48 & 1.17 & 1.09 & 1.01 & 1.38 \\
\hline & $(0.26)$ & $(0.33)$ & $(0.23)$ & $(0.25)$ & $(0.47)$ & $(0.46)$ & $(0.82)$ & $(1.18)$ \\
\hline controls & no & yes & no & yes & no & yes & no & yes \\
\hline module dummies & yes & yes & yes & yes & yes & yes & yes & yes \\
\hline Mean of dep. var & 0.501 & 0.500 & 0.742 & 0.741 & 0.350 & 0.356 & 0.728 & 0.728 \\
\hline Pseudo-R^2 & 0.036 & 0.062 & 0.056 & 0.048 & 0.037 & 0.066 & 0.026 & 0.089 \\
\hline \# of obs. & 916 & 914 & 643 & 641 & 926 & 924 & 471 & 471 \\
\hline
\end{tabular}

Table 9.3: Logistic regressions on racial/ethnic background categories.

Note: Omitted category is white, non-hispanic. Robust standard error in parentheses.

$*: p=0.1, * *: p=0.05, * * *: p=0.01$.

\begin{tabular}{|c|c|c|c|c|c|c|c|c|}
\hline \multirow[t]{2}{*}{ Dependent var } & \multicolumn{2}{|c|}{ Dominated } & \multirow{2}{*}{$\begin{array}{l}\text { R. av. } \\
\text { (3) }\end{array}$} & \multirow{2}{*}{$\begin{array}{l}\text { - gains } \\
\text { (4) }\end{array}$} & \multicolumn{2}{|c|}{ R. av. - losses } & \multicolumn{2}{|c|}{ Loss av. } \\
\hline & (1) & (2) & & & $(5)$ & (6) & (7) & (8) \\
\hline \multirow[t]{2}{*}{ log income (odds ratio) } & 0.99 & $0.84^{*}$ & 1.14 & 0.93 & $1.19^{* *}$ & $1.33^{* * *}$ & 1.24 & 1.05 \\
\hline & $(0.07)$ & $(0.08)$ & $(0.11)$ & $(0.11)$ & $(0.09)$ & 0.14 & $(0.16)$ & $(0.19)$ \\
\hline controls & no & yes & no & yes & no & yes & no & yes \\
\hline module dummies & yes & yes & yes & yes & yes & yes & yes & yes \\
\hline Mean of dep. var & 0.501 & 0.500 & 0.742 & 0.741 & 0.350 & 0.356 & 0.728 & 0.728 \\
\hline Pseudo-R^2 & 0.026 & 0.062 & 0.007 & 0.048 & 0.040 & 0.066 & 0.010 & 0.089 \\
\hline \# of obs. & 916 & 914 & 643 & 641 & 926 & 924 & 471 & 471 \\
\hline
\end{tabular}

Table 9.4: Logistic regressions on log income.

Note: Robust standard error in parentheses. ${ }^{*}: p=0.1,{ }^{* *}: p=0.05,{ }^{* * *}: p=0.01$. 


\begin{tabular}{|c|c|c|c|c|c|c|c|c|}
\hline \multirow[t]{2}{*}{ Dependent var } & \multicolumn{4}{|c|}{ Dominated } & \multicolumn{4}{|c|}{ R. av. - gains } \\
\hline & (1) & $(2)$ & (3) & $(4)$ & $(5)$ & (6) & (7) & (8) \\
\hline \multirow[t]{2}{*}{ math correct (odds ratio) } & 0.80 & - & - & 0.76 & 1.11 & - & - & 1.11 \\
\hline & $(0.17)$ & - & - & $(0.16)$ & $(0.31)$ & - & - & $(0.31)$ \\
\hline \multirow[t]{2}{*}{ college math course } & - & $1.33^{*}$ & - & 1.34 & - & 0.93 & - & 0.84 \\
\hline & - & $(0.21)$ & - & $(0.27)$ & - & $(0.19)$ & - & $(0.21)$ \\
\hline \multirow[t]{2}{*}{ bachelor's degree } & - & - & 1.19 & 1.04 & - & - & 1.08 & 1.18 \\
\hline & - & - & $(0.20)$ & $(0.23)$ & - & - & $(0.24)$ & $(0.32)$ \\
\hline controls & yes & yes & yes & yes & yes & yes & yes & yes \\
\hline Mean of dep. var & 0.508 & 0.508 & 0.508 & 0.508 & 0.747 & 0.747 & 0.747 & 0.747 \\
\hline Pseudo-R^2 & 0.056 & 0.057 & 0.056 & 0.059 & 0.036 & 0.036 & 0.036 & 0.037 \\
\hline \# of obs. & 884 & 884 & 884 & 884 & 621 & 621 & 621 & 621 \\
\hline
\end{tabular}

Table 9.5: Logistic regressions of FOSD choices and risk averse choices in gains domain on math-skills related variables. Note: Robust standard error in parentheses.

$$
*: p=0.1, * *: p=0.05, * * *: p=0.01 \text {. }
$$

\begin{tabular}{|c|c|c|c|c|c|c|c|c|}
\hline \multirow[t]{2}{*}{ Dependent var } & \multicolumn{4}{|c|}{ R. av. - losses } & \multicolumn{4}{|c|}{ Loss av. } \\
\hline & (1) & (2) & (3) & (4) & (5) & (6) & (7) & (8) \\
\hline \multirow[t]{2}{*}{ math correct (odds ratio) } & $1.45^{*}$ & - & - & $1.52^{* *}$ & 0.82 & - & - & 0.84 \\
\hline & $(0.30)$ & - & - & $(0.32)$ & $(0.29)$ & - & - & $(0.32)$ \\
\hline \multirow[t]{2}{*}{ college math course } & - & 0.84 & - & 0.82 & - & 1.21 & - & 1.61 \\
\hline & - & $(0.14)$ & - & $(0.17)$ & - & $(0.33)$ & - & $(0.53)$ \\
\hline \multirow[t]{2}{*}{ bachelor's degree } & - & - & 0.90 & 0.95 & - & - & 0.85 & 0.64 \\
\hline & - & - & $(0.16)$ & $(0.21)$ & - & - & $(0.24)$ & $(0.23)$ \\
\hline controls & yes & yes & yes & yes & yes & yes & yes & yes \\
\hline Mean of dep. var & 0.344 & 0.344 & 0.344 & 0.344 & 0.735 & 0.735 & 0.735 & 0.735 \\
\hline Pseudo- $R^{\wedge} 2$ & 0.064 & 0.062 & 0.062 & 0.066 & 0.081 & 0.081 & 0.081 & 0.085 \\
\hline \# of obs. & 893 & 893 & 893 & 893 & 453 & 453 & 453 & 453 \\
\hline
\end{tabular}

Table 9.6: Logistic regressions of risk averse choices around zero and in losses domain on education and math-skills related variables. Note: Robust standard error in parentheses.

$$
*: p=0.1, * *: p=0.05, * * *: p=0.01 \text {. }
$$


The tables show that between most subgroups of respondents, the differences in FOSD violations are insignificant. In particular, Table 9.1 indicates no effect at all of the decisionmaker's gender, not even on the revealed degrees of risk aversion in any of the three subsets of lottery outcomes that we consider. Before turning to the other explanatory variables of interest, notice another indication of the lack of explanatory power of any background variable - the Pseudo- $R^{2}$ value lies below 0.1 for all regressions.

Age has a mildly adverse effect on behavior (see Table 9.2), in the sense that older participants make weakly more FOS-dominated choices. But the significance of the effect goes away when squared age is included, or when other controls are included, so we regard it as an non-robust effect.

Nonwhites makes significantly fewer FOS-dominated choices, and in particular the hispanic population in the sample has a much lower frequency of FOSD violations. Table 9.3 shows that their frequency is at less than 50 percent of the frequency of the omitted category (white, non-hispanic). Furthermore, columns (3), (5) and (7) give a more detailed account of these differences, indicating that hispanics exhibit a similar behavior when the offered lotteries that lie in the negative domain, but that they are much less risk averse around zero and in the domain of gains. This corresponds closely to the comparison between the estimated preferences in Figure 5, where the two groups showed strong differences for payoffs around zero and higher.

The respondent's income is only weakly correlated with the frequency of FOSD violations, as Table 9.4 shows. Columns (5) and (6) indicate that higher-income participants behave less riskseeking in the losses domain than lower-income participants (again, in close correspondence to the model estimates - see Figure 6), but this difference does not carry over to a significant and robust reduction in FOS-dominated choices.

Perhaps the most surprising result is that none of the variables that may proxy for analytical skills yields a significant reduction in the number of FOSD violations. Neither the ability to answer our three mathematics questions correctly, nor their general educational background and their mathematics-related background are found to be significantly correlated with the number of $A$ and $D$ choices — see Tables 9.5 and 9.6. Figures 7-9 show that this finding is consistent with the absence of differences between the respondents' risk attitudes. 


\section{References}

Abdellaoui, M., C. Barrios, and P.P. Wakker (2007), "Reconciling revealed utiltiy with introspective preference: Experimental arguments based on Prospect Theory," Journal of Econometrics 138, 356-378.

Barberis, N., and M. Huang (2004), "Preferences with frames: A new utility specification that allows for the framing of risks," mimeo, Yale University.

Barberis, N., M. Huang, and R.H. Thaler (2006), "Individual preferences, monetary gambles, and stock market participation: A case for narrow framing," American Economic Review 96, 1069-1090.

Battalio, R.C., J.H. Kagel, and K. Jiranyakul (1990), "Testing between alternative models of choice under uncertainty: Some initial results," Journal of Risk and Uncertainty $3,25-50$.

Bell, D.E. (1985), "Disappointment in decision making under uncertainty," Operations Research 33, 1-27.

Benartzi, S., and R.H. Thaler (1995), "Myopic loss aversion and the equity premium puzzle," Quarterly Journal of Economics 110, 73-92.

Benjamin, D.J., S.A. Brown, and J.M. Shapiro (2005), "Who is 'behavioral'? Cognitive ability and anomalous preferences," mimeo, Harvard University.

Bowman, D., Minehart, D., and M. Rabin (1999), "Loss aversion in a consumtionsavings model," Journal of Economic Behavior and Organization 38, 155-178.

Camerer, C.F. (1989), "An experimental test of several generalized utility theories," Journal of Risk and Uncertainty 2, 61-104.

Camerer, C.F. (1995), "individual decision making," in Handbook of Experimental Economics, Kagel, J., and A.E. Roth, editors, Princeton: Princeton University Press.

Cox, J.C., and V. Sadiraj (2006), "Small- and large-stakes risk aversion: Implications of 
concavity calibrations for decision theory," Games and Economic Behavior, forthcoming.

de Finetti, B. (1974), Theory of Probability, Vol. 1, New York.

Diecidue, E., Schmidt, U., and P.P. Wakker (2004), "The utility of gambling reconsidered," Journal of Risk and Uncertainty 29, 241-259.

Diecidue, E., and P.P. Wakker (2002), "Dutch books: Avoiding strategic and dynamic complications, and a comonotonic extension", Mathematical Social Sciences 43, 135-149.

Dohmen, T., A. Falk, D. Huffman, U. Sunde, J. Schupp, and G.G. Wagner (2005), "Individual risk attitudes: Evidence from a large, representative, experimentally-validated survey", IZA Discussion Paper 1730.

Dohmen, T., A. Falk, D. Huffman, U. Sunde (2007), "Are risk aversion and impatience related to cognitive ability?", IZA Discussion Paper 2735.

Frederick, S. (2005), "Cognitive reflection and decision making," Journal of Economic Perspectives 19, 25-42..

Gneezy, U., and J. Potters, "An experiment on risk taking and evaluation periods," Quarterly Journal of Economics 112, 631-645.

Holt, C.A., and S.K. Laury (2002), "Risk aversion and incentive effects," American Economic Review 92, 1644-1655.

Kahneman, D., and D. Lovallo (1993), "Timid choices and bold forecasts: A cognitive perspective on risk-taking," Management Science 39, 17-31.

Kőszegi, B., and M. Rabin (2007), "Reference-dependent risk attitudes," American Economic Review, forthcoming.

Langer, T., and M. Weber (2001), "Prospect theory, mental accounting, and differences in aggregated and segregated evaluation of lottery portfolios," Management Science 47, 716-733. 
Loomes, G., and R. Sugden (1986), "Disappointment and dynamic consistency in choice under uncertainty," Review of Economic Studies 53, 271-282.

Rabin, M. (2000a), "Risk aversion and expected utility theory: A calibration theorem," Econometrica 68, 1281-1292.

Rabin, M. (2000b), "Diminishing Marginal Utility of Wealth Cannot Explain Risk Aversion," in: Choices, Values, and Frames, Kahneman, D., and A. Tversky, editors, New York: Cambridge University Press.

Rabin, M., and R.H. Thaler (2001) "Anomalies: Risk aversion," Journal of Economic Perspectives 15, 219-232.

Read, D., Loewenstein, G., and M. Rabin (1999), "Choice bracketing," Journal of Risk and Uncertainty 19, 171-197.

Redelmeier, D., and A. Tversky (1992), "On the framing of multiple prospects," Psychological Science 3, 191-193.

Rubinstein, A. (2006), "Dilemmas of an economic theorist," Econometrica 74, 865-883.

Safra, Z., and U. Segal (2005), "Calibration results for non-expected utility theories," mimeo, Tel-Aviv University and Boston College.

Starmer, C. (2000), "Developments in non-expected utility theory: The hunt for a descriptive theory of choice under risk," Journal of Economic Literature 38, 332-382.

Thaler, R.H., A. Tversky, D. Kahneman, and A. Schwartz, "The effect of myopia and loss aversion on risk taking: An experimental test," Quarterly Journal of Economics $112,647-661$.

Tversky, A., and D. Kahneman (1981), "The framing of decisions and the psychology of choice," Science 211, 453-458.

Tversky, A., and D. Kahneman (1992), "Advances in prospect theory: Cumulative representation of uncertainty," Journal of Risk and Uncertainty 5, 297-323. 
Wakker, P.P. (2005), "Formalizing Reference Dependence and Initial Wealth in Rabin's Calibration Theorem." mimeo, Erasmus University, Rotterdam. 